\title{
LARP6C orchestrates post-transcriptional reprogramming of gene expression during hydration to promote pollen tube guidance
}

\author{
Elodie Billey ${ }^{1,2,3^{*}}$, Said Hafidh ${ }^{*}$,@, Isabel Cruz-Gallardo ${ }^{5,6}$, Celso G. Litholdo $\mathrm{Jr}^{1,2}$, \\ Viviane Jean ${ }^{1,2}$, Marie-Christine Carpentier ${ }^{1,2}$, Claire Picart ${ }^{1,2}$, Vinod Kumar ${ }^{4}$, \\ Katarina Kulichova ${ }^{4}$, Eric Maréchal ${ }^{7}$, David Honys ${ }^{4}$, Maria R. Conte ${ }^{6}$, Jean-Marc \\ Deragon,1,2,8@ and Cécile Bousquet-Antonelli1,2,@
}

${ }^{1}$ CNRS LGDP-UMR5096, 58 Av. Paul Alduy 66860 Perpignan, France.

2 Université de Perpignan Via Domitia, LGDP-UMR5096, 58 Av. Paul Alduy 66860 Perpignan, France.

${ }^{3}$ Present address: TOTAL RC, 2 place Jean Millier- Arche Nord Coupole/Regnault, 92078 Paris La Défense Cedex, France

${ }^{4}$ Laboratory of Pollen Biology, Institute of Experimental Botany of the Czech Academy of Sciences, Rozvojovà 263, 16502 Prague 6, Czech Republic.

${ }^{5}$ Randall Centre for Cell and Molecular Biophysics, King's College London, New Hunt's House, Guy's Campus, London SE1 1UL, UK.

${ }^{6}$ Present address: Department fo Chemistry, King's College London, Britannia House, 7 Trinity St, London SE1 1DB, UK

${ }^{7}$ Laboratoire de Physiologie Cellulaire et Végétale, UMR 5168 CNRS, CEA, INRAE, Université Grenoble Alpes, IRIG, CEA Grenoble, Grenoble, France.

${ }^{8}$ Institut Universitaire de France, 1 rue Descartes, 75231 Paris Cedex 5 France

*: These authors contributed equally to the work

Corresponding authors:

cecile.antonelli@univ-perp.fr, hafidh@ueb.cas.cz, jean-marc.deragon@univ-perp.fr

Short title: AtLARP6C in pollen tube guidance

One-sentence summary: The RNA-Binding protein LARP6C is a key player in polarized pollen tube growth in Arabidopsis via post-transcriptional regulation of a subset of mRNAs in dry pollen and during the fertilization process.

The authors responsible for distribution of materials integral to the findings presented in this article in accordance with the policy described in the instruction for Authors (www.plantcell.org) are: Dr. C. Bousquet-Antonelli (cecile.antonelli@univ-perp.fr), Dr. Said Hafidh (hafidh@ueb.cas.cz) and Pr. JM Deragon (jean-marc.deragon@univperp.fr).

\section{ABSTRACT}

Increasing evidence suggests that post-transcriptional regulation is a key player in the transition between mature pollen and the progamic phase (from pollination to fertilization). Nonetheless, the actors in this mRNA-based gene expression reprogramming are poorly understood. We demonstrate that the evolutionarily conserved RNA-binding protein (RBP) LARP6C is necessary for the transition from dry pollen to pollen tubes and the guided growth of pollen tubes towards the ovule in Arabidopsis thaliana. In dry pollen, LARP6C binds to transcripts encoding proteins that function in lipid synthesis and homeostasis, vesicular trafficking, and polarized cell growth. LARP6C also forms cytoplasmic granules that contain the poly(A) binding 
protein and possibly represent storage sites for translationally silent mRNAs. In pollen tubes, loss of LARP6C negatively affects the quantities and distribution of storage lipids, as well as vesicular trafficking. In Nicotiana benthamiana leaf cells and in planta, analysis of reporter mRNAs designed from the LARP6C target MGD2 provided evidence that LARP6C can shift from a repressor to an activator of translation when the pollen grain enters the progamic phase. We propose that LARP6C orchestrates the timely post-transcriptional regulation of a subset of mRNAs in pollen during the transition from the quiescent to active state and along the progamic phase to promote male fertilization in plants.

\section{INTRODUCTION}

2 In the cytoplasm, mRNAs coated with RNA-binding proteins (RBPs) in the form of ribonucleoprotein particles (mRNPs) are in balance between translation, storage, and decay. Translationally silent mRNPs can be stored and protected from decay in cytosolic aggregates and released when their protein product is needed. In response to developmental or environmental cues, the fine-tuning between mRNA decay rate or storage will have a direct impact on translation and hence gene expression. RBPs play a crucial role in this fine-tuning, enabling gene-specific regulation through binding to their mRNA targets.

LA and Related Proteins (LARPs) form a large family of eukaryote RBPs that share the La Motif (LAM), a structured RNA-binding domain. LARPs are classified into five distinct subfamilies, including the LARP6 group (Bousquet-Antonelli and Deragon, 2009). In most LARPs, an RNA Recognition Motif (RRM1) is found immediately after the LAM and forms a bipartite RNA-binding unit called the La-module (Maraia et al., 2017). While the LAM is usually very well conserved between subfamilies, the RRM1s are specific to each subgroup (Bousquet-Antonelli and Deragon, 2009; Maraia et al., 2017). Members of the LARP6 subfamily are found in Stramenopiles, Chlorophytes, plants, invertebrates, and vertebrates and are characterized by the La-module and by

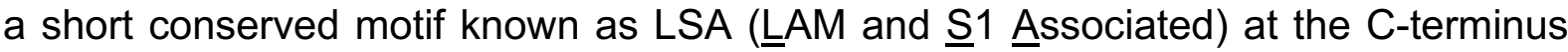
(Bousquet-Antonelli and Deragon, 2009) that mediates protein-protein interactions (Cai et al., 2010b; Manojlovic et al., 2017; Vukmirovic et al., 2013; Weng et al., 2009). In mammals, one of the functions of LARP6 is to regulate the synthesis of type I collagen, a heterotrimer composed of two $\alpha 1$ and one $\alpha 2$ subunits whose assembly and correct secretion are dependent upon the coordinate translation of their transcripts at the endoplasmic reticulum (ER) (Zhang and Stefanovic, 2016). The expression of 
a stem loop in their 5'-UTRs (Cai et al., 2010a; Martino et al., 2015). LARP6 either stores or stabilizes the collagen mRNA transcripts in a translationally silent form by tethering them to vimentin filaments where they can further be activated for translation or subjected to decay (Challa and Stefanovic, 2011). Alternatively, LARP6 docks the mRNAs to non-muscle myosin filaments (Cai et al., 2010b; Manojlovic et al., 2017) and either recruits STRAP (Serine Threonine kinase Receptor Associated Protein) (Vukmirovic et al., 2013) or RNA helicase A (RHA) via its LSA (Manojlovic et al., 2017), which respectively function as a repressor and activator of translation. Through this mechanism, LARP6 orchestrates the coordinated translation of $\alpha 1$ and $\alpha 2$ collagen transcripts at the ER. More recently, human LARP6 was found to regulate the localisation and translation of ribosomal protein (RP) mRNAs to cell protrusions, enhancing RP synthesis, ribosome biogenesis, and overall protein synthesis in migratory cells (Dermit et al., 2020). Furthermore, in zebrafish (Danio rerio), both egg activation (Hau et al., 2020).

While LARP6 proteins are generally encoded by a single gene, vascular plant proteins are encoded by 3 to 6 genes and are of three evolutionary types named $6 \mathrm{~A}$, 6B, and 6C (Merret et al., 2013b). Whilst members of the 6A subgroup most closely resemble LARP6 from other eukaryotes, in addition to a reorganized La-module, Band C-type orthologs carry a PABP-interacting motif 2 (PAM2). Arabidopsis thaliana has three AtLARP6 genes, one of each type, which we will refer to as $L A R P 6 A, B$ and $C$ throughout this manuscript.

We previously demonstrated that LARP6C directly associates with the poly $(A)$ binding protein (PABP) in plants, which is consistent with the presence of a PAM2 motif and that the finding that its La-module binds to oligo $\left(\mathrm{U}_{20}\right)$ homopolymers in vitro. In addition, in onion (Allium cepa) epidermis, LARP6C accumulates in the cytoplasm, the nucleoplasm, and the nucleolus. Strikingly, LARP6C redistributes to stress granules upon hypoxia, where it associates with mRNP aggregates (Merret et al., 2013b). These data support the view that Arabidopsis LARP6C is an RNA-binding protein that is likely involved in mRNA post-transcriptional regulation.

In flowering plants, the haploid male gamete (pollen grain) consists of a vegetative cell that encases two sperm cells, which are connected (through a so-called cytoplasmic connexion) with the vegetative cell nucleus to form the male germ unit. A characteristic feature of angiosperm sexual reproduction is that sperm cells are non- 
motile and must be carried simultaneously to the ovule by a pollen tube (Johnson and Preuss, 2002). Upon adhesion to the stigma, the male vegetative cell hydrates, germinates, and grows into a tube that delivers the two sperm cells to the ovule. The pollen tube is a highly polarized and fast tip-growing cell whose growth directionality is determined by various perceived cues. It is first guided in the gynoeceum by female sporophytic tissue in the pistil (preovular guidance) and then, upon exit from the transmitting tract, it navigates in gametophytic tissues guided by ovule-emitted signals (ovular guidance). Ovular cues guide the pollen tube alongside the funiculus to the micropyle. Upon reaching the ovule, the pollen tube stops growing and bursts to free the sperm cells that will fuse respectively with the egg cell (to form the embryo) and the central cell (to form the endosperm), hence completing the fertilization process (Higashiyama and Takeuchi, 2015).

In the pollen tube, upon reception and transduction of extracellular female signals, a complex cellular process targets the cell wall synthesis machinery at the site of perception, hence permitting the delivery of macromolecules at site of extension and the directional growth of the tube. This requires tip-localized receptors, the flow of ions, intracellular trafficking of signalling molecules and proteins, but also vesicular trafficking, cytoskeleton-dependent transport, and novel cell wall formation through exocytosis (Feng et al., 2018; Hafidh et al., 2014; Higashiyama and Takeuchi, 2015). Pollen tube growth also requires changes in the pattern of gene expression that at least in part rely on post-transcriptional mechanisms. Several studies have suggested that all mRNAs required for germination and pollen tube growth are already present in the mature pollen grain and maintained in a translationally silent state until the progamic phase (Hafidh et al., 2018; Honys et al., 2000; Honys and Twell, 2004; Scarpin et al., 2017). Nonetheless, how male gene expression is post-transcriptionally regulated to permit correct pollen guidance is largely unknown, and the mRNAregulating factors required for proper ovular guidance of pollen are currently unclear.

In the present study, we further explored the role of LARP6C and determined that it is a pollen-specific factor required for male fertility during the progamic phase. We provide evidence that loss of LARP6C affects lipid synthesis and homeostasis as well as vesicular trafficking. Moreover, our experimental results support the notion that LARP6C orchestrates the balance between the translation, storage, and decay of its mRNA clients through direct binding, thus intervening in the guided growth of the pollen tube. 


\section{RESULTS}

LARP6C and its RNA-binding domain are required for male fertility at the pollen tube guidance step

100 Immunoblotting and high-throughput transcriptomic analyses (Klepikova et al., 2015) showed that, unlike LARP6A and 6B, LARP6C is almost exclusively expressed in pollen grains and pollen tubes (Figure 1A and Supplemental Figure S1). We therefore investigated its role in male fertility using two Arabidopsis T-DNA insertion mutants, larp6c-3 and larp6c-4. Immunoblotting showed that both alleles are loss-of-function (lof) mutants (Figure 1B). To establish whether larp6c is a gametophytic mutation, we monitored the transmission efficiency (TE) through the female $\left(\mathrm{TE}^{f}\right)$ and the male $\left(\mathrm{TE}^{\mathrm{m}}\right)$ gametophytes by reciprocal crosses with wild type and heterozygous larp6c-3/+ or larp6c-4/+ mutants (Figure 1C). Both alleles were transmitted normally through the female gametophyte $\left(\operatorname{TE}^{f} 6 c-3=100 \%\right.$ and $\left.6 c-4=96.7 \%\right)$, whereas their transmission through the male was significantly reduced, with $6 c-3$ showing TEm $=74.1 \%$ and $6 c-4$ $\mathrm{TE}^{\mathrm{m}}=68.2 \%$ (Figure $1 \mathrm{C}$ ). This suggests that male but not female fertility is compromised by the loss of LARP6C function.

There are different stages at which the male gametophyte could be deficient, including pollen development, germination, pollen tube growth or guidance, and sperm delivery (Johnson and Preuss, 2002). We monitored these pollen stages in larp6c-3, $6 c-4$, and wild-type plants (Figure 1D-F and Supplemental Figure S2A-E). Assessment of male gametophyte maturation by DAPI staining revealed that $10 \%$ of the $6 c-3$ and $6 c-4$ mutant pollen grains did not complete their maturation and were arrested at the bicellular stage, compared to less than 3\% in wild type (Supplemental Figure S2A).

We next monitored germination and pollen tube growth during the early and late steps after hydration (in vitro) or pollination (in vivo and semi in vivo [SIV]) (Palanivelu and Preuss, 2006) and Methods) (Figure 1D and Supplemental Figure S2). In vitro, larp6c-3 showed some defects in germination, with over $70 \%$ of ungerminated pollen grains after 30 min incubation versus 60\% for wild type and an increased frequency of pollen tube burst. Moreover, at $30 \mathrm{~min}$, larp6c-3 showed less than 5\% elongated pollen tubes versus $20 \%$ in the wild type, e.g. pollen tubes with lengths over three times that of mature pollen grains. In vivo, the situation appeared more dramatic, with a very high levels of ungerminated larp6c-3 pollen at 15 and $30 \mathrm{~min}$ after pollination of over 80 and 
$12950 \%$, respectively, compared to wild-type pollen, which showed less than $10 \%$ 130 ungerminated pollen after $15 \mathrm{~min}$ incubation. Moreover, in vivo, at $30 \mathrm{~min}$, larp6c-3 131 pollen tubes seemed to be more prone to bursting than wild type (Figure 1D). These 132 germination and pollen tube growth defects were likely due to delays rather than full 133 arrest, since 7 and 24 hours after pollination (Supplemental Figure S2B, C and E) or 4 134 hours after hydration (Supplemental Figure S2D), the density and length of larp6c pollen tubes were similar to those of wild type.

We next investigated the requirement for LARP6C for pollen tube guidance and ovule targeting competence (Johnson and Preuss, 2002). We set up SIV experiments using pistils from ms1 plants, a male-sterile mutant with normal pistil function (van der Veen and Wirtz, 1968), to assess the ability of larp6c-deficient pollen tubes to be guided through the pistil tissues towards wild-type ovules for fertilization (Figure 1E, top panel and Supplemental Figure S3A). Whereas wild-type pollen targeted $80 \%$ to $100 \%$ of the ovules (with a median value of around $90 \%$ ), both larp $6 c-3$ and $6 c-4$ pollen showed a significant reduction, targeting 50 to $70 \%$ of wild-type ovules (with a median value of $60 \%$ ). This failed pollen tube guidance phenotype was rescued by the $\mathrm{N}$ - and C-terminal LARP6C fusion constructs (YFP-LARP6C and LARP6C-tRFP) (Figure 1E and Supplemental Figure S3B-C). In competition assays, irrespective of the order of pollination or the side of the stigma in which the pollen grains were dusted on, larp6c3 and $6 c-4$ pollen also showed a clear deficiency in ovule targeting, with a maximum targeting efficiency of $20 \%$ compared to the expected $50 \%$ rate (Supplemental Figure S3A and D). This further supports the notion that the observed guidance phenotype directly relates to larp6c deficiency.

We next monitored the ability of the larp6c and larp6c-complemented pollen to target ovules in planta (Figure 1E-F and Supplemental Figure S2E). We hand pollinated $m s 1$ pistils and following aniline blue staining, scored the number of targeted and non-targeted ovules. Aniline blue stains callose-like polysaccharides contained in pollen tube walls, and after exposure to UV emits yellow-green fluorescence that allows visualization of pollen tubes even after penetration in the ovule. Here again the larp6c deficient pollen tubes displayed only $60 \%$ ovule targeting efficiency versus the $80 \%$ average observed in wild-type pollinated pistils (Figure 1E, bottom panel). The

160 targeting efficiency by the (larp6c-3; LARP6C-tRFP) and (larp6c-3; YFP-LARP6C) 161 complemented lines recovered to a wild-type frequency (Figure 1E). Considering that 1624 hours after pollination, the density and length of mutant pollen tubes were similar to 
163 those of wild type (Supplemental Figure S2E), it appears unlikely that the ovule targeting deficiency of larp6c pollen was due to delays in germination or pollen tube elongation. Nonetheless, to completely rule out this possibility, we monitored the type of ovule targeting defects at 4, 8, and 24 hours after pollination (HAP) in vivo. We observed and scored two types of behaviour: type I: if pollen tube attraction and reception were normal and type II: if pollen tube attraction was defective. We did not observe a situation where attraction was normal but reception defective. Consistent with previous experiments, we found that larp6c-deficient pollen tubes showed a significant increase in type II behaviour compared to wild type (Figure 1F). At 24 HAP, while some $40 \%$ of the ovules remain unfertilized in pistils pollinated with larp $6 c-3$ or 4 mutant pollen due to attraction defects, only $5 \%$ of the ovules were not fertilized when pollination was conducted with wild-type pollen (Figure 1F).

Considering that LARP6A mRNA is highly expressed in pollen (3 to 4 times more than LARP6C mRNA in mature pollen grain, Supplemental Figure S1A), we wondered whether LARP6A and LARP6C could at least partially act redundantly. In backcross experiments, larp6a-1 loss-of-function pollen (Supplemental Figure S3F) displayed over $95 \%$ transmission efficiency, and the transmission efficiency of (larp6c3; larp6a-1) was not significantly different from that of single larp6c-3 mutant pollen (82.7\%) (Supplemental Figure S3F). We also ran in vivo pollination assays with wildtype, larp6c-3, or (larp6c-3; larp6a-1) pollen and scored fertilization behaviours (Supplemental Figure S3G). Consistent with the results of backcross experiments, the absence of LARP6A did not affect fertilization in the wild-type or larp6c null background.

Finally, to confirm that larp6c loss of function specifically affects male fertility, we examined the capability of larp6c-3 and 6c-4 mutant ovules to attract wild-type pollen tubes by SIV and in vivo assays and found no significant difference from wild type (Supplemental Figure S4). A detailed description of these experiments can be found in the legend of Supplemental Figure S4.

We previously demonstrated that LARP6C is an RNA-binding protein and that its La-module is sufficient to bind RNA homopolymers in vitro (Merret et al., 2013b). We hence wondered whether the RNA-binding function of LARP6C is necessary for its role in pollen tube guidance. Since for LARPs, including human LARP6, which carry a bipartite La-Module, the LAM is absolutely necessary to bind RNA both in vitro and

196 in vivo (Martino et al., 2015; Cai et al., 2010a), we constructed a truncated LARP6C 
deleted of its La Motif ( $L A R P 6 C \Delta-L A M)$. We then stably expressed this deletion mutant construct in the larp6c-3 and larp6c-4 backgrounds under the control of the LARP6C native promoter (Supplemental Figure S3C). In vivo fertilization assays showed that pollen expressing LARP6C $\triangle \mathrm{LAM}$ had the same pollen tube guidance defects as larp6c loss-of-function pollen, suggesting that $L A R P 6 C \Delta-L A M$ cannot complement the larp6c3 lof phenotype and that the La-Module RNA-binding motif is essential for LARP6C function in pollen tube guidance (Figure 1F).

In summary, our results support the notion that LARP6C is required for male fertility at least in part at the progamic phase through its function in pollen germination and during pollen tube guidance and does not appear to act redundantly with LARP6A. Moreover, LARP6C function in pollen tube guidance requires an intact La-Module, supporting the notion that this physiological role involves the RNA-binding activity of LARP6C.

LARP6C forms dynamic cytoplasmic granules in mature pollen grains and in pollen tubes

213 To better understand the cellular roles of LARP6C, we monitored its subcellular distribution across pollen maturation and during pollen tube growth (Figure 2A and Supplemental Figure S5). In microspores, LARP6C was only detected in the nucleolus. At the bicellular stage, LARP6C was much more generally distributed in the cytoplasm of the vegetative cell, as well as in the vegetative and generative cell nuclei. At the tricellular stage, the subcellular distribution of LARP6C was similar to that at the bicellular stage, with possibly reduced amounts of LARP6C in the sperm cell nucleus compared to other subcellular compartments. In mature pollen grain, LARP6C displayed a complex localization pattern. It was clearly present in vegetative cell cytoplasm but transitioned from a diffuse pattern to a granular, aggregate-like pattern (Figure 2A and Supplemental Figure S5). In addition to this granular distribution, LARP6C appeared to concentrate around the membrane surrounding the vegetative cell nucleus, the sperm cells and the cytoplasmic connection linking the sperm cell 1 to the vegetative cell nucleus.

To determine more precisely the distribution of LARP6C in mature pollen grains, we performed colocalization experiments between LARP6C-tRFP and various markers (Figure 2B). The histone H2B-GFP fusion protein, expressed under the control of the tomato (Solanum lycopersicum) pollen-specific LAT52 promoter (Twell et al., 1989), 
was used to label the vegetative cell nucleus, thereby demonstrating the absence of LARP6C in this compartment. GEX2 is a sperm cell-expressed transmembrane protein (Mori et al., 2014) that labels the sperm cell membranes and the cytoplasmic connection between sperm cell 1 and the vegetative cell nucleus (Brownfield et al., 2009; McCue et al., 2011; Mori et al., 2014). Confocal analyses showed that LARP6C and GEX2 displayed distinct distributions (Figure 2B). The tRFP signal surrounded the GFP-GEX2 signal decorating the outer membrane of the male germ unit but not the membrane connecting the two sperm cells (the inner membrane), supporting the notion that LARP6C is excluded from the sperm cells and therefore only resides in the vegetative cell cytoplasm in mature pollen.

We next inquired on the nature of LARP6C cytoplasmic foci (Figure 2B). Eukaryotic cells contain many types of membrane-less cytoplasmic aggregates composed of translationally silent mRNAs and RBPs (Buchan, 2014). So far, only two types of mRNP granules have been identified in plant cells: processing bodies ( $p-$ bodies) and stress granules (Weber et al., 2008). Plant p-bodies are - as in other eukaryotes - characterized by the presence of actors of the general mRNA turnover process, such as the DCP1/DCP2 decapping holoenzyme, the XRN4 exoribonuclease, or the deadenylation complex. Stress granules, on the contrary, contain factors belonging to the translational machinery, including the poly $(A)$ binding protein (Weber et al., 2008). First clues on the nature of LARP6C aggregates observed in mature pollen were derived by colocalization experiments with specific markers of p-bodies and stress granules, namely DCP1 and PABP, respectively. We used a YFP-tagged DCP1 protein expressed from its own upstream genomic sequences (Merret et al., 2013a). Although YFP-DCP1 displayed a punctuate distribution in the vegetative cell cytoplasm, similar to LARP6C, no obvious colocalization of DCP1 and LARP6C was observed. Of note, DCP1 accumulation appeared to be restricted to the vegetative cell cytoplasm, at least in mature pollen, and contrary to LARP6C, it did not localize at the periphery of the sperm cells or the vegetative nucleus (Figure 2B).

The Arabidopsis genome contains seven genes (PAB2 to 8 ) encoding canonical PABPs, including PAB3, 5, 6 and 7 (Belostotsky and Meagher, 1996; Belostotsky, 2003; Honys and Twell, 2004), whose transcripts accumulation is restricted to pollen. We fused the genomic sequence of the PAB5 gene (the most highly expressed of the pollen specific PABP genes) to a YFP tag under the control of its own upstream sequences. In the vegetative cell cytoplasm, the YFP-PAB5 protein displayed a pattern 
identical to that of the LARP6C protein in that it accumulated in foci surrounding the sperm cells and was excluded from the vegetative nucleus, but contrary to LARP6C, it was also present in the cytoplasm of sperm cells.

Finally, in pollen tubes, LARP6C was clearly excluded from the sperm cells and restricted to vegetative cell cytoplasm. In the cytoplasm, LARP6C signals showed various degrees of aggregate patterns, with foci appearing to be organized along a longitudinal string-like structure (Figure 2A and Supplemental Figure S5C). To explore the possibility that LARP6C foci could bind to the cytoskeleton structure, we coexpressed LARP6C-tRFP with GFP-AtTUB6 (which labels microtubules) in Nicotiana benthamiana epidermis cells (Ambrose and Cyr, 2007), GFP-FABD2 (Voigt et al., 2005), or LifeACT-GFP (Cvrčková and Oulehlová, 2017) (which label actin microfilaments; Figure 2C and Supplemental Movie S1). While LARP6C did not significantly co-localize with actin microfilament markers, it co-localized with GFPtubulin (Figure 2C and Supplemental Movie S1). Not only did the strings formed by LARP6C foci overlap with tubulin filaments, but LARP6C foci also appeared to move along microtubules, as revealed by time series observations (Supplemental Movie S1).

Finally, as an additional exploration of possible redundancy, we ran colocalization experiments between LARP6C and LARP6A (Supplemental Figure S5B, C). We previously showed that, when transiently expressed in onion epidermis cells, LARP6C localized to stress granules (together with PABP), while LARP6A did not. Consistently, in mature pollen grain, we observed that LARP6C formed foci, while LARP6A did not (Supplemental Figure S5C). Additionally, LARP6A and 6C showed distinct subcellular distributions in pollen tubes, with 6A mainly accumulating in sperm cell cytoplasm while 6C was excluded from the sperm cells (Supplemental Figure $\mathrm{S} 5 \mathrm{C})$.

In summary, our data indicate that LARP6C shows a dynamic subcellular distribution across pollen maturation. We found that in mature pollen and pollen tubes, LARP6C is restricted to the vegetative cell cytoplasm and is present in aggregates that could possibly associate with microtubules.

\section{RIP-seq pulldown reveals LARP6C mRNA targets in mature pollen grain}

296 The presence of LARP6C in cytoplasmic aggregates, which contain the poly(A) binding protein, together with our previously published data (Merret et al., 2013b), strongly support the notion that at the cellular level, LARP6C likely acts to control mRNA fate 
299 in pollen. In addition, since its RNA-binding domain is necessary to fulfil its role in 300 pollen tube guidance, we reasoned that the identification of its mRNA clients would 301 help reveal how LARP6C controls male fertilization at the molecular level. We hence 302 sought to identify its mRNA targets using an unbiased RNA immunoprecipitation and 303 sequencing (RIP-seq) strategy. We conducted the RNA immunoprecipitation 304 procedure from complemented larp6c-3 lof mature pollen grains expressing a $305 \mathrm{PRO}_{6 \mathrm{c}}$ :LARP6C-FlagHA tagged version (6C-FH hereafter) (Supplemental Figure S3B, 306 C) and from wild-type pollen grains as a negative control. After assessing the efficiency 307 of the immunoprecipitation through immunoblotting (Supplemental Figure S6A), RNAs 308 were extracted from the input and eluate fractions and subjected to RNA-seq. After 309 mapping and filtering of the dataset (see Supplemental Methods), a RPKM (read per 310 kilobase per million mapped reads) value was calculated for each protein-coding gene. 311 The replicates were found to be reproducible (Supplemental Figure S6B), enabling us 312 to build a table with a mean normalized value per gene and filter out those with less 313 than 1 RPKM value in one of the four samples (input or eluate from wild type or 6C$314 \mathrm{FH}$ ) (Supplemental Data Set S1). Using such criteria, we retained a list of 7356 315 expressed transcripts.

316 We first filtered out transcripts based on input fraction values, retaining mRNAs 317 that are not differentially expressed (DE) and show a fold change between 6C-FH and 318 wild-type < 1.2 in order to select for transcripts whose levels did not vary between 319 control and 6C-FH inputs. Then, from this trimmed list, we selected mRNAs that are 320 DE (FDR 0.01) and accumulated to a higher extent in the eluate fraction of 6C-FH 321 compared to wild type (Figure 3A). Next, we selected mRNAs that were enriched by 322 the immunoprecipitation procedure, by selecting those with a $R_{6 C-F H}(R E$ is the eluate 323 over input ratio) value $>1$. To further increase stringency and reveal mRNAs that are 324 most likely to be direct targets of LARP6C, we compared the RE $\mathrm{WT}_{\mathrm{T}}$ to the RE $\mathrm{E}_{6 \mathrm{C}-\mathrm{FH}}$ and, 325 based on the repartition of the values (Supplemental Figure S6C and D) selected 326 transcripts with an ( $\left.\mathrm{RE}_{6 \mathrm{C}-\mathrm{FH}} / \mathrm{RE}_{W T}\right)$ value of 3 or more (Figure $\left.3 \mathrm{~B}\right)$. Through the above 327 series of filters, we identified a high-confidence list of 115 genes encoding direct or indirect mRNA targets of LARP6C (Supplemental Data Set S1).

329 No gene ontology (GO) term was found to be significantly enriched amongst the 330115 mRNAs identified as potential LARP6C targets. Nonetheless, a gene-to-gene 331 analysis shows that the majority of LARP6C targets encode factors likely to be involved 332 in polarized cell growth and male fertilization. This includes protein involved in 
endomembrane system trafficking and synthesis, signalling factors and kinases

334 (Grebnev et al., 2017; Samaj et al., 2006) and most strikingly proteins involved in lipid metabolism and homeostasis control (Ischebeck, 2016) (Figure 3C, Supplemental

336 Data Set S1). Also, we found that some LARP6C targets are known actors of pollen

337 gametogenesis, pollen germination, pollen tube growth and guidance, or tip-polarized

338 growth, such as the grown of roots or neurons (Supplemental Data Set S1).

Loss of LARP6C affects lipid metabolism and the endomembrane system during pollen tube growth

342 In angiosperms, lipids are crucial for male fertilization. Pollen tube elongation depends on lipids synthesized and stored in pollen grain before desiccation or produced on the way from pistil to ovule (Ischebeck, 2016). Amongst the list of LARP6C targets are actors involved in fatty acid synthesis, di- and triacylglycerol (TAG) synthesis and homeostasis, whose control were previously found to be necessary for correct pollen tube growth (Botté et al., 2011; Pleskot et al., 2012; Zheng et al., 2018). This includes genes encoding fatty acid synthesis factors (FAD2 (Botella et al., 2016), ACX4 (Khan et al., 2012) and AtLPEAT2 (Stålberg et al., 2009)); DGAT1, which catalyzes the first step of TAG synthesis (Zhang et al., 2009); DYRKP-2A (Schulz-Raffelt et al., 2016) and MPK6 kinases, which regulate TAG production (Zheng et al., 2018); SDP1, a TAG lipase involved in membrane lipid homeostasis control (Kelly et al., 2013); and MGD2, which catalyzes the synthesis of monogalactosyldiacylglycerol (MGDG) (Botté et al., 2011).

Considering that LARP6C might control the expression of genes encoding proteins involved in glycerolipid metabolism and homeostasis at the posttranscriptional level, we tested lipid accumulation and synthesis in the absence of LARP6C in mature pollen grains and pollen tubes (Figure 4). First, we visualized lipid droplets (LDs), which consist of a phospholipid monolayer surrounding a core of neutral lipids such as TAG and fatty acid steryl esters, by staining with Bodipy 505/515 (which stains neutral lipids) and Nile Red (which stains non-polar and neutral lipids)

362 (Figure 4A-B). In larp6c-3 mature pollen grain, we did not detect any significant 363 difference in staining compared to wild-type grains (Figure 4A). We then germinated and grew wild-type and larp6c-3 pollen tubes in vitro and visualized LD accumulation at 2 and 4 h post germination (Figure 4B). At 2h after gemination, 77 out 132 (58\%) observed pollen tubes showed fewer LDs compared to wild type, and at $4 \mathrm{~h}, 84 \%$ ( $\mathrm{n}$ 
$=100 / 119$ ) of pollen tubes showed an abnormal distribution of LDs. In $72 \%$ of the cases, LDs were absent from apical and sub-apical regions and were only detected in the shank, and $12 \%$ showed an overaccumulation and aggregation of lipids at the tip. Such aberrant distribution was never observed amongst the wild-type pollen tubes ( $\mathrm{n}$ = 109). This phenomenon was even more pronounced when pollen tubes were grown through a pistil explant, with $100 \%$ of larp6c-3 pollen tubes showing an atypical distribution of LDs (Figure 4C, SIV). These results support the notion that lipid synthesis and/or distribution in pollen tubes is perturbed in the absence of LARP6C.

We next explored the impact of the downregulation of lipid synthesis on pollen tube guidance in the absence of LARP6C. Amongst the LARP6C mRNA targets, MGD2 encodes a pollen specific (our data and (Qin et al., 2009)) MGDG synthase (Botté et al., 2011). In situ labelling of Arabidopsis pollen tube membranes previously showed the presence of digalactosyldiacylglycerol (DGDG) (which are synthesized from MGDG), and the chemical inhibition of the MGD enzymatic pathway with galvestine-1 reduces MGDG content and downregulates pollen tube elongation rates (Botté et al., 2011), supporting a role for MGD in male fertilization.

We reasoned that if one of the molecular bases of the guidance defect of larp6c relates to the misregulation of MGD2 mRNA fate and expression, the specific inhibition of its enzymatic activity and downregulation of MGDG/DGDG production with galvestine-1 should alleviate or aggravate the larp6c lof phenotype. We hence monitored pollen tube elongation rates and pollen tube guidance in the presence of galvestine-1 by SIV assays (Figure 4D, E). Consistent with a previous report (Botté et al., 2011), we observed that galvestine-1 application reduced pollen tube elongation in wild-type pollen as well as larp6c-3 mutant pollen (Figure 4D). However, when we monitored pollen tube guidance efficiency, wild-type pollen tubes were only slightly or not affected at all, whereas pollen tubes of larp6c mutants showed a considerable decrease in ovule targeting in the presence of galvestine-1 (Figure 4E). Under these conditions, only 30 to $60 \%$ of ovules were targeted by larp6c-pollen tubes, whereas the mock-treated samples showed 60 to $70 \%$ targeting. In some pistil explants, larp6c pollen tubes showed no ovule targeting at all in the presence of the MGD inhibitor, a situation that was never observed with larp6c-3 pollen tubes in the absence of the MGD inhibitor (Figures 1E-F and 4E). Collectively, these results support a synergic effect between the loss-of-function of LARP6C and the pharmacological inhibition of MGD2 synthase activity on pollen tube guidance but not on pollen tube elongation. 
LARP6C also binds mRNA encoding factors involved in endosome functioning and vesicular trafficking. Brefeldin $A$ (BFA) is a fungal toxin known to perturb endomembrane system trafficking, particularly at the level of retrograde Golgi to ER trafficking and to induce ectopic ER-Golgi fusions known as BFA bodies (Tse et al., 2006). We therefore monitored larp6c-3 pollen tube sensitivity to BFA compared to wild type (Figure 5). After $1 \mathrm{~h}$ activation on germination medium, the pollen tubes were exposed to BFA and the number and size of BFA bodies scored over time through FM 4-64 staining. As soon as $10 \mathrm{~min}$ after exposure to BFA, 6c-3 pollen tubes showed increased number of BFA bodies and a dramatic increase in size compared to wildtype 17 min after treatment. At this time point, ectopic BFA-particles were also bigger in the mutant than in wild type. These results indicate that pollen tubes deprived of LARP6C are hypersensitive to pharmacological perturbation of endosomal trafficking.

\section{LARP6C directly binds to the 5'-UTRs of its mRNA targets} We next sought to understand how LARP6C recognises and influences the fate of its mRNA targets through binding. RNA-binding proteins often bind to primary sequence motifs shared between their mRNA targets to regulate their fate. Considering that cis regulatory elements are mostly located in the 5' and/or 3' untranslated regions (UTRs) of transcripts, we looked for primary sequence motif(s) shared between the UTRs of the putative LARP6C targets. Using the Araport database, we found and retrieved the 5'- and 3'-UTRs for 112 mRNAs and ran a discriminative motif search using a MEME search (at the MEME suite portal (https://meme-suite.org/)). Whereas no significant motif could be detected in the 3'-UTRs of LARP6C mRNA targets, we identified two regions we named $A$-box ( $E^{\text {value: } 5.5 E^{-99}}$ ) and $B-b o x$ ( $E^{\text {value: } 9.3 E^{-91}}$ ) motifs that are highly enriched in the 5'-UTRs of LARP6C mRNA targets (Figure 6A). As a control, we ran an identical motif search using a set of 112 randomly chosen 5 '- and 3'-UTRs from genes expressed in pollen according to our transcriptomic analyses. We found no significant enrichment for an A- or a B-type region, supporting the idea that these sequence motifs are specific for mRNAs that are in a complex with LARP6C.

The $A$ motif is a purine-rich $21 \mathrm{nt}$ sequence with a clear dominance of $A$ over $G$ nucleotides, while the $B$ motif is a pyrimidine-rich $21 \mathrm{nt}$ sequence with a majority of $U$

432 ( $T$ in DNA) over $C$ repeats (Figure 6A, Supplemental Data Set S1). A closer 433 examination of sequences that contain the $A$ and/or $B$ consensus sequence revealed 434 two major categories for each box type. In particular, sequences harbouring the A motif 
are mainly composed of either $A$ or $A G / A A G$ repeats, whilst sequences sharing the $B$ consensus motif contain either U or UC/UUC repeats (Supplemental Data Set S1). Amongst the 112 identified 5'-UTRs, 70 (62.5\%) bear the A motif, 74 (66\%) the B motif, and $49(43.7 \%)$ carry both (Supplemental Figure S6E).

The presence of motifs shared by transcripts that co-immunoprecipitated with LARP6C in vivo suggests that one or both of these boxes mediate the direct binding of LARP6C. To explore this hypothesis, we tested the RNA-binding unit of LARP6C, the La-module, for its ability to interact with these boxes in vitro (Figure 6B-E and Supplemental Figure S7). We designed two oligonucleotide sequences for the A motifs called A1 and A2, representing the "A-rich" and the "AG/AAG-rich" types, respectively (Supplemental Figure S7A). Analogously, B1 and B2 were designed for the B motifs exemplifying the "U-rich" and "UC/UUC-rich" types, respectively (Figure 6B). As a control, we designed oligos B3 and B4, representing C- or CU/CCU-rich sequences, respectively. A recombinant version of the LARP6C La-module, identical to the one used in Merret et al. (Merret et al., 2013b), was expressed in E. coli as previously described and its ability to bind the $A$ and $B$ type oligo RNAs was quantified by isothermal titration calorimetry (ITC) and electrophoretic mobility shift assay (EMSA) (Figure 6B-E and Supplemental Figure S7). In the ITC experiments, serial titration of LARP6C La-module with RNA oligos B1 and B2 generated (in both cases) a profile that could be fitted to a sigmoid-shaped binding curve centred around a $1: 1$ stoichiometry, with a dissociation constant in the low micromolar range (Kds of $2.8 \mu \mathrm{M}$ and 3.7 $\mu \mathrm{M}$, respectively, indicating direct binding of LARP6C La-module to B1 and B2 type motifs (Figure 6B and Supplemental Table S1). The binding mode of LARP6C Lamodule for the B1 and B2 oligos is similar (but with higher affinity) to that previously observed for an U20 homopolymer (Supplemental Table S1 and (Merret et al., 2013b)). On the contrary, no binding of oligos B3, B4, A1, or A2 to the recombinant LARP6C domain was detected by ITC under the same experimental conditions (Figure 6D, Supplemental Figure S7A and Supplemental Table S1).

To confirm the ITC results, parallel EMSAs were performed using RNA oligos labelled at the $5^{\prime}$ end with $\gamma^{32} \mathrm{P}$ to monitor RNA-protein complex formation by native gel electrophoresis. Experiments were conducted in the presence or absence of tRNA competitor. For oligos showing the ability to form a complex with La-module, the fraction of bound RNA was plotted against the protein concentration and the 
dissociation constant determined (Figure 6C). In agreement with the ITC results, EMSA confirmed that B1 and B2 oligos associate with the LARP6C La-module, with estimated dissociation constants in the low micromolar range ( $\mathrm{Kds}$ of $0.8 \mu \mathrm{M}$ and 1.9 $\mu \mathrm{M}$, respectively), again suggesting a high affinity of LARP6C La-module towards B1 and B2 oligos (Figure 6C). The binding behaviour of B2 oligo was very similar to what we observed for an oligo $\left(\mathrm{U}_{20}\right)$ (Merret et al., 2013b), whereas B1 showed a slightly higher binding affinity. Assays with oligos $A 1, A 2, B 3$, oligo $\left(C_{20}\right)$ and to a lesser extent with B4 were hindered by samples being retained in the wells of the gel at high protein concentration (Figure 6E and Supplemental Figure S7B), an issue most likely linked to protein and/or protein/RNA non-specific aggregation (Hellman and Fried, 2007). Despite changing various experimental conditions, this issue could not be overcome. The presence of a tRNA competitor marginally helped in the case of B4, albeit association with LARP6C was observable only at high protein concentrations and a Kd could not be calculated, as the binding curve did not reach a plateau (Figure 6E).

Collectively, these experiments demonstrate that LARP6C La-module binds to oligos B1 and B2 with high affinity and with a similar association mechanism observed with oligo $\left(U_{20}\right)$. B1, which contains three stretches of $U$ residues, one of which is 6 uridines long, appeared to bind slightly more tightly than $B 2$ and $U_{20}$. Altogether, these results indicate that the LARP6C La-module strongly binds to $B$ type motifs and does not associate with A type motifs, at least in vitro, and that it displays low affinity for B4. We hence propose that in vivo, LARP6C directly binds (at least) to mRNAs that carry a B1 or B2-type box in their 5'-UTRs to execute post-transcriptional regulation on its mRNA targets.

\section{LARP6C is not required for the accumulation of its mRNA targets in mature pollen grain}

To gain a global view of the role of LARP6C in the accumulation of its mRNA targets, we monitored the polyadenylated transcriptome of mature pollen grains in the absence of LARP6C. Pollen grains were collected from wild-type and larp6c-3 plants grown together in a greenhouse (see Supplemental Methods), total RNAs were extracted from the pollen, and the polyadenylated fraction was subjected to next generation sequencing. Following mapping and filtering of the reads (see Supplemental Methods), we retained a list of 7399 transcripts with at least 1 RPKM as reliably expressed from both Col-0 and larp6c-3 (Supplemental Figure S8, Supplemental Data Set S2). We 
then used a DE-seq pipeline (FDR 0.05) to find genes whose transcripts differentially accumulate in the mutant background and retained those with a fold change of at least 1.5 between wild type and larp6c-3. We found 2174 genes that were up- or downregulated in larp6c-3 pollen compared to the wild type (Figure 7A). Monitoring the fold changes of these transcripts between wild type and (larp6c-3; 6C-FH) in the input fraction of the RIP-seq samples, we observed that they are centred around 1 (Figure $7 \mathrm{~B})$. This suggests that the expression of the LARP6C-FlagHA transgene in the larp6c3 background fully complemented the larp6c-3 mutation and restored a wild-type expression level, demonstrating that the LARP6C-FlagHA translational fusion is functional and that the mis-regulation of the larp $6 c-3$ pollen transcriptome is biologically significant and a direct consequence of the loss of LARP6C function. We then asked

513 if the steady-state levels of LARP6C-bound mRNAs are affected in larp6c-3 pollen 514 grains. Of the 115 targets, 96 were detected in the RNA-seq data, and only 15 of these 515 differentially accumulated in the larp6c-3 background (Figure 7C, Supplemental Data 516 Set S2). Of these, ten were downregulated by 1.5- to 2.8-fold and five were upregulated by 1.5- to 1.7-folds in larp6c-3 (Figure 7D). These observations suggest that, at least in dry pollen, LARP6C is not required for maintaining steady-state levels of its mRNA targets.

\section{LARP6C controls the expression of a reporter transcript containing a functional} 5'-UTR B-box motif

To gain a mechanistic insight into the impact of LARP6C binding to its mRNA targets, we performed transient assays to monitor the expression of a YFP reporter gene under the control of a modified 5'-UTR belonging to MGD2, a LARP6C mRNA target that carries a potential bona fide B-box and no A-box motif (Supplemental Data Set S1). We then respectively replaced the native $B-b o x$ by two sequences that we demonstrated in vitro are strongly bound (B1-box) or not bound at all (B3-box) by the LARP6C La-module (Figure 6). We inserted the modified 5'-UTR sequences downstream of the CaMV35S promoter and fused to coding sequence of YFP (B1-YFP and $B 3-Y F P$ ) in a plant binary vector (Figure 8A). These YFP-containing plasmids were transiently expressed in Nicotiana benthamiana leaves individually or co-infiltrated with

534 CaMV35S promoter. Three days post infiltration, leaves were collected and YFP mRNA and protein levels assessed (Figure 8B and C). Quantitative analyses showed 
536 that when the LARP6C protein was present, B1-YFP transcript levels increased by 537 two-fold (Figure 8B). Conversely, co-expression of LARP6C with the B3-YFP construct 538 did not significantly affect B3-YFP mRNA levels (Figure 8B). Remarkably, the presence 539 of LARP6C significantly reduced YFP protein accumulation when expressed from the 540 B1 but not the B3 construct (Figure 8C). We also monitored the subcellular distribution of the tRFP-LARP6C fusion protein following agroinfiltration. Similar to findings for mature pollen grains (Figure 2) and onion epidermis cells following stress exposure, LARP6C formed foci in the cytoplasm of $N$. benthamiana leaves (Figure 8D). Since in pollen grains and onion epidermis cells, LARP6C foci contained the poly(A) binding protein, we reason that LARP6C also likely aggregates into mRNP granules in $N$. benthamiana.

\section{LARP6C controls native MGD2 protein levels and distribution in pollen tubes}

We next asked whether LARP6C controls the mRNA and proteins levels of their target genes using MGD2 mRNA as an example. We cloned the MGD2 genomic locus (starting at position - 579 from ATG) including its native B-box motif in fusion with the YFP coding region that we placed at the C-terminus and transformed this chimeric gene into the wild-type background. We then screened pollen and pollen tubes from 22 primary transformants and selected line 6, which we crossed to larp6c-3 homozygous plants. In the F2 generation, we selected plant homozygous for the larp6c-3 allele that expressed heterozygous MGD2-YFP. We then performed microscopic observations of wild type and larp6c-3 pollen that was heterozygous for the MGD2-YFP transgene. Pollen grains were placed on in vitro pollen germination agarose pad medium and YFP signals monitored in mature pollen grain (dry pollen), pollen at 15 and 30 minutes after hydration (Figure 9A), and pollen tubes at the 4 hour time point (Figure 9B). Strikingly, both in wild type and larp6c-3, mature pollen grains show very low levels of YFP signal, which intensified over time upon hydration and in pollen tubes.

To confirm this finding and to assess the role of LARP6C in MGD2 protein accumulation, we quantified fluorescence intensity in dry pollen and at $1 \mathrm{~h}$ and $4 \mathrm{~h}$ after activation. As shown in Figure 9C, MGD2-YFP levels drastically increased in pollen tubes $1 \mathrm{~h}$ after activation in both the wild type and mutant. Nonetheless, compared to wild type, larp6c-3 dry pollen showed higher levels of fluorescence, a situation opposite that observed in $1 \mathrm{~h}$ pollen tubes, which showed reduced MGD2-YFP signals in the 
570 absence of LARP6C. Using specific antibodies (Awai et al., 2001), we made similar 571 observations for endogenous MGD2 accumulation, finding significantly more protein in 572 pollen tubes than in dry pollen. Moreover, the loss of LARP6C function induced 573 overaccumulation of MGD2 in mature pollen and underaccumulation in pollen tubes 574 compared to wild type (Figure 9C right panel). Next, RT-qPCR assays revealed that 575 the mRNA levels of MGD2-YFP and endogenous MGD2 did not significantly vary 576 before and after pollen hydration and that loss-of-function of LARP6C did not affect mRNA levels (Figure 9D). This is consistent with previously published transcriptomic data showing that endogenous MGD2 mRNA levels did not significantly vary between dry pollen and pollen tubes after $30 \mathrm{~min}$ or at $4 \mathrm{~h}$ activation in vitro (Qin et al., 2009).

Beyond an impact on protein levels, the loss of LARP6C function also appeared to affect MGD2-YFP distribution along the pollen tube (Figure 9B). In wild type, MGD2YFP signal showed a diffuse cytosolic pattern, with the signal intensity distributed along a gradient decreasing from the shank toward the tip in almost $94 \%$ ( $n=124)$ of the pollen tubes. In the absence of LARP6C, the gradient distribution of MGD2-YFP was lost in $59 \%(n=109)$ of the observed pollen tubes, which showed a uniform distribution of the signal that also reached the pollen tube tip.

\section{DISCUSSION}

589 We report here that LARP6C, an evolutionarily conserved RNA-binding protein, is essential for male fertility, acting during gametogenesis and germination and especially during the progamic phase, during which it is necessary for the directional growth of pollen tubes.

\section{The role of LARP6C in vesicular trafficking during male fertilization}

595 The anisotropic growth of the pollen tube, which takes place exclusively at the apical 596 dome, requires vast amounts of secretory vesicles delivered at the tip via cytoplasmic 597 streaming. Golgi-derived secretory vesicles supply cell wall material as well as material 598 for plasma membrane extension at the site of growth. Vesicles also deliver 599 transmembrane proteins, such as receptor kinases, which are inserted into the apical 600 plasma membrane through exocytosis (Grebnev et al., 2017). Indeed, the exocyst secretory complex was found to be essential for the specification of plasma membrane

602 nanodomains that define sites for the directional growth of pollen tubes (Li et al., 2010; 603 Synek et al., 2017, 2006; Vukašinović and Žárský, 2016). We found that larp6c-pollen 
604

605

606

607

608

609

610

611

612

613

614

615

616

617

618

619

620

621

622

623

624

625

626

627

628

629

630

631

632

633

634

635

636

637

tubes are hypersensitive to Brefeldin-A and identified several transcripts encoding components of the vesicular system as LARP6C mRNA clients, including factors involved in the trafficking of ER vesicles, constituents of the vacuolar trafficking system HOPS (Takemoto et al., 2018), components and regulators of the ESCRT (Endosomal Sorting Complex Required for Transport) (Reyes et al., 2014), and a subunit of the exocyst complex (Supplemental Data Set S1). We hence propose that the pollen tube guidance defects of larp6c mutants are in part the consequence of abnormal vesicular trafficking.

\section{LARP6C is necessary for lipid homeostasis during male fertilization}

To optimize polar tip growth, pollen has evolved a unique lipid composition. In particular, pollen grains produce extra-plastidial glycerolipids and store fatty acids and TAGs in LDs (Ischebeck, 2016). LDs are necessary for gametogenesis, pollen grain maturation, viability, and germination. They also might be required for pollen tube guided growth, as supported by the finding that MPK6 is a regulator of their synthesis involved in pollen tube guidance (Zhang et al., 2009; Zheng et al., 2018). The exact role of LDs in pollen tubes is not currently clear, but it has been proposed that TAGs could be synthesized in the shank of the pollen tube and transported to the tip in the form of LDs to deliver membrane components (Ischebeck, 2016). In line with this idea, we report that LDs are distributed from shank to tip in wild-type pollen tubes (Figure 4). Hence, the role of LARP6C in pollen tube guidance could also involve maintaining adequate LD levels, distribution, and possibly composition, as it can specifically bind mRNAs encoding fatty acid, TAG, phospho-, and glycerolipid synthases (FAD2, ACX4, TAG1, LPEAT2, MGD2) for lipases such as SDP1, which is involved in storage lipid breakdown (Kelly et al., 2013), and for regulators of their synthesis, such as DYRKP2A (Schulz-Raffelt et al., 2016) and MPK6 kinases (Zheng et al., 2018).

MGD2 is one of the three Arabidopsis synthases that catalyse the last step of MGDG synthesis. Consistent with the finding that galactolipids are unique and major constituents of plastid membranes, MGD1 and MGD3 were previously found to localize inside chloroplasts and at the periphery of plastids, respectively, which are consistent with a protein embedded in the outer envelope membrane. The subcellular distribution of MGD2 was more difficult to interpret than that of its two counterparts, as it was detected in the cytosol (Awai et al., 2001). Here, in the absence of any plastid, we also report that MGD2 is present in the cytosol of the pollen tube and that it is distributed 
from shank to tip along a decreasing gradient that is lost in the absence of LARP6C. These observations uncover an unsuspected role for cytosolic MGD2, which could have the ability to produce MGDG outside plastids and possibly transport its substrate DAG to the site of MGDG synthesis. Since MGDG is the substrate for DGDG, which was detected at the periphery of pollen tubes (Botté et al., 2011), MGD2 could participate to pollen tube membrane expansion. Alternatively, as DAG is also a substrate for TAG production, MGD2 may also transport this substrate to the sites of LD production and therefore participate to the guidance process. The changes in MGD2 protein levels and distribution along pollen tubes could hence be another reason for the observed guidance defect of the larp6c mutants.

On top of vesicular trafficking and lipid dynamics, LARP6C likely controls other cellular processes required for the directed growth of pollen tubes. Indeed, several LARP6C targets encode actors in processes involved in pollen tube elongation and guidance, such as signalling factors and kinases, cell wall modification players, or ion transporters. Moreover, some LARP6C clients were experimentally found to be involved in anisotropic cell elongation. These include, the transcription factor TFIIB1, which controls guided pollen tube growth (Zhou et al., 2013); TUB4, a structural microtubule component required for normal hypocotyl guidance and elongation (Yu et al., 2015); or the $\alpha / \beta$ hydrolase-domain protein WAV2, which is involved in directional

\section{Molecular functions of LARP6C during male fertilization} LARP6C is an RNA-binding protein that physically interacts with PABP, a master regulator of mRNA translation and decay (Merret et al., 2013b). Here we demonstrated that LARP6C at least in part co-localizes with PABP in mature pollen grain.

663 Furthermore, a LARP6C protein deficient in its RNA-binding domain (La-module) was unable to restore LARP6C's function in pollen tube guidance. We hence propose that LARP6C acts at the molecular level to control gene expression by regulating mRNA fate in the cytoplasm.

We provided strong evidence that LARP6C directly interacts with mRNAs harbouring B-boxes of the B1 or B2 type in their 5'-UTRs (i.e. at least 52 of the 115 transcripts identified by RIP-seq (Supplemental Data Set S1)). For some transcripts, only A-boxes were identified in the 5'-UTR. This might have occurred because we were only able to retrieve a partial 5'-UTR sequence, and inadvertently missed sequence 
regions that could contain the B-box, but it is also possible that some LARP6C RIP targets are in complex with this RBP but not through direct binding.

In N. benthamiana leaf epidermis, LARP6C appeared to control the levels of its mRNA targets as well as the amount of the corresponding translated proteins. LARP6C promoted the overaccumulation of YFP mRNA that carried its recognition sequence, specifically a B1 type B-box motif (Figure 6). We propose that at least in somatic cells, this overaccumulation is the result of LARP6C-mediated protection from cytoplasmic decay, rather than an action at the transcriptional level. Surprisingly, our results also show that this increase in mRNA levels is associated with a decrease in the corresponding YFP protein levels (Figure 8). This suggests that LARP6C could, while protecting its mRNA targets, dampen their translation. Alternatively, LARP6C could control protein stability, but since it is unlikely to interact with the reporter YFP protein (the only protein encoded by the reporter gene we designed), we deem this hypothesis unlikely. In eukaryotic cells, one common feature of mRNP granules is that they contain translationally silent mRNAs that are capable of (re)-entering translation in response to the appropriate signals and protect these mRNAs from decay (Buchan, 2014). Hence, the presence of LARP6C in foci that we propose are mRNP storage granules also rather favours the first hypothesis.

The mRNA stabilization effect of LARP6C binding observed in transient $N$. benthamiana assays is in contrast with the observation that in pollen grains, LARP6C does not seem to control its targets' steady-state levels. In pollen, as in animal gametes, mRNAs are highly stable, suggesting that in the male gametophyte, the mRNA decay machineries, as well as the translational apparatus, have poor activity (Ylstra and McCormick, 1999; Hafidh et al., 2018). This assumption is consistent with our observation that DCP1, a core constituent of the mRNA decapping complex, localises to foci in mature pollen grains and that this localisation is thought to be linked to quenched DCP1 activities. Indeed, work conducted in animal cells proposed that when components of the mRNA decay machineries are present in p-bodies, they are catalytically inactive (Hubstenberger et al., 2017). The discrepancies between the results from transient assays in $N$. benthamiana leaf cells and RNA-seq of pollen grains could hence be the consequence of differences in how the mRNA decay complex operates between sporophytic and reproductive cells.

In vivo, we propose that LARP6C plays a dual role in controlling of the levels of translated proteins encoded by its targets' transcripts. Using MGD2 mRNA as a model, 
we indeed showed that whilst antagonizing MGD2 protein accumulation in mature pollen grains, LARP6C promotes its translation after gemination without affecting MGD2 mRNA levels (Figure 9). Although the possibility that LARP6C regulates the amount of MGD2 protein post-translationally cannot be ruled out at this stage, we believe that it is unlikely, as discussed for the YFP reporter protein. This implies that LARP6C shifts from a repressor to an activator role in translation. This could be the result of a differential pattern of post-translation modifications that might allow LARP6C to recruit different sets of accessory proteins to its target mRNAs before and after pollen hydration, resulting in a mode switch from repression to activation.

In 2017, Scarpin et al. reported that like MGD2 mRNA, SK14 mRNA is translationally silent in mature pollen grain and reactivated at germination. They also found that SK14 mRNA aggregates in sub-cytoplasmic bodies that also contain the pbody component DCP1 (Scarpin et al., 2017). Here, we also demonstrated the existence of foci in mature pollen grain containing proteins that function in mRNA regulation (LARP6C and PAB5), which we believe also contain mRNAs. mRNP granules exist in most eukaryotes and in many cell types and are of different types based on their protein content. However, a common feature is that they store and protect translationally silent mRNPs until their protein product is needed. We therefore propose that a large portion of mRNAs whose expression is necessary for male fertilization are stored in mature pollen grains in the form of mRNP aggregates until their protein products are required during the progamic phase, as supported by previous findings (Hafidh et al., 2018), and that LARP6C is one of the trans-acting factors that orchestrate this process (see Supplemental Figure $\$ 9$ for a summary model).

An intriguing observation from the current study is that LARP6C appears to be required for the distribution of the protein encoded by one of its client mRNAs, MGD2, along the pollen tube. We presently have no conclusive proof that LARP6C does not act at the protein level to participate in this gradient distribution. Nonetheless, considering that LARP6C is an mRNA-binding protein, we hypothesize that the defective distribution of MGD2 in larp6c is the consequence of the aberrant regulation of its mRNA. We propose that LARP6C transports the translationally silent mRNAs present in mRNP granules to the appropriate cytoplasmic location where the protein is then translated and required. The existence of such localized translation events following the transport of silent mRNAs is a conserved and widespread feature of 
eukaryotes, including plants, where few examples of mRNAs targeted to the surface of the ER, chloroplasts, or mitochondria have been reported (Tian et al., 2020). In the vast majority of cases, mRNAs are transported by cytoskeletal motors in the form of silent mRNPs (Bullock, 2011; Eliscovich and Singer, 2017). Consistent with a possible role in mRNA delivery, our data support the notion that LARP6C, in association with mRNP granules, likely navigates along microtubules in growing pollen tubes. Considering the phenotypes of larp6c-pollen, we propose that mRNA movement and localized translation are necessary for the polarized growth of pollen tubes and hence plant reproduction.

The role of LARP6 proteins in mRNA control might be evolutionarily conserved Interestingly, a recent study by Dermit et al. demonstrated that human LARP6 acts in migrating cells to navigate mRNAs encoding ribosomal proteins (RPs) along microtubules to transport them from the cell body to protrusions; these cellular migrating fronts act as translation hotspots. This function of human LARP6 in the spatiotemporal control of RP mRNA translation is necessary for cell proliferation and migration (Dermit et al., 2020). The physiological roles of zebrafish LARP6 differ from that of Arabidopsis LARP6C, as it participates in oocyte development, chorion formation, and egg activation whilst it is not involved in male fertilization. Nevertheless, the authors suggest that zebrafish LARP6 could function in chorion formation by regulating mRNA metabolism in microvilli, i.e., fine cell protrusions extending from both the developing oocytes and granulosa cells (Hau et al., 2020). It is interesting that LARP6 proteins, which are highly conserved eukaryotic RBPs, seem to have acquired distinct physiological roles during eukaryote evolution but could have retained their molecular mode of action in mRNA transport and translational control.

\section{METHODS}

\section{Plant material and growth conditions}

Arabidopsis thaliana ecotype Columbia-0 (Col-0) was used as the wild-type reference. The larp6c-3 (SAIL_268E02) (McElver et al., 2001) and larp6c-4 (WiscDsLox293296invG4) (Woody et al., 2007) lines were respectively ordered from the NASC and ABRC stock centres. Genotyping primers are shown in Supplemental Data Set S3.

773 Stable transgenic lines were obtained using the Agrobacterium tumefaciens-based 
774 floral dip technique (Clough and Bent, 1998). For in vitro culture, surface sterilized seeds were sown on synthetic Murashige \& Skoog (MS) medium at $2.20 \mathrm{~g} / \mathrm{L}(1 / 2 \mathrm{MS})$ with $0.8 \%$ agar and stored for $48 \mathrm{~h}$ at $4^{\circ} \mathrm{C}$ in the dark before being grown under continuous light $\left(50-60 \mu \mathrm{E} \cdot \mathrm{m}^{-2} \cdot \mathrm{s}^{-1}\right)$ at $20^{\circ} \mathrm{C}$. Plants in soil cultures were grown in growth chambers under a $16 \mathrm{~h}$ light $\left(100 \mu \mathrm{E} \cdot \mathrm{m}^{-2} \cdot \mathrm{s}^{-1}\right) / 8 \mathrm{~h}$ dark cycle at $20^{\circ} \mathrm{C}$ with approximately $75 \%$ humidity. See Supplemental Methods for growth conditions used to collect mature pollen for RNA-seq and RIP-seq experiments.

\section{Cloning and plasmids}

All LARP6C-containing fusion proteins (YFP, TagRFP, and FLAGHA) were expressed under the control of the upstream genomic sequences (spanning region -1181 to -1 from ATG) of the LARP6C gene (AT3G19090 locus). To obtain the LARP6C-tRFP and LARP6C-FLAGHA fusions, we isolated total genomic DNA from Col-0 and PCR amplified the $L A R P 6 C$ genomic region starting from nucleotide -1181 from the ATG to the last nucleotide before the stop codon using primers 543 and 544 (Supplemental Data Set S3). The PCR product was inserted at sites Kpnl and Nhel upstream of either the tagRFP or FLAG-HA tag into a pCAMBIA1300-based plant binary vector expressing the HPTII (hygromycin) resistance gene, giving rise to vectors pEB16 and pEB13. To obtain the YFP-LARP6C expressing binary vector, the genomic region spanning nucleotides -1181 to -1 from the ATG of the LARP6C gene was PCR amplified with primers 974 and 975 from vector pEB13 and cloned at sites Sacl and Kpnl upstream of a Gateway entry cassette into a pPZP221 (Hajdukiewicz et al., 1994) based plant binary vector carrying the $A A C 1$ (gentamycin) resistance gene, giving rise to vector $\mathrm{p} 788$. The $L A R P 6 C$ coding genomic region was PCR amplified from total genomic DNA with primers 976-977 and cloned at sites Spel-EcoRV downstream of the YFP tag located between the AttL1 and L2 Gateway donor sites, giving rise to plasmid p793. The plant binary vector p795 was obtained through a Gateway cloning reaction between p788 and 793 . The PAB5 genomic sequence (-1966 from the ATG to the last nucleotide before the stop codon) was PCR amplified from total genomic

803 DNA with primers eb3 and eb4 and cloned using the restriction endonucleases Xbal 804 and BamHI into vector CTL579 (pCAMBIA-1300) upstream of the GFP tag, giving rise 805 to plasmid pEB3. To prepare transgenic lines expressing the YFP-LARP6C $\triangle \mathrm{LAM}$ 806 protein, we transformed larp6c-3 or larp6c-4 plants with plasmid p844. The 
LARP6C $\triangle L A M$ CDS codes for a LARP6C protein deleted of the region spanning amino acids 137-227 and was obtained as follow. We PCR amplified the regions of the coding sequence from +1 to +411 from ATG (primers 1197 and 1198) with primer 1198, which also adds a 3'-tail extension of $20 \mathrm{nt}$ complementary to region 684-704 of the CDS (located just downstream to the LAM). A second PCR product was generated with

812 primers 1199-1200, starting from position 684 to the stop codon. To fuse both products,

813 we ran a third PCR amplification with primers 1201-1200 and an equimolar mixture of 814 products 1 and 2 as a matrix. The resulting fragment corresponds to the full-length 815 LARP6C CDS deleted of its LAM and carrying Xbal and EcoRV restriction sites at its $8165^{\prime}$ and $3^{\prime}$ ends, respectively. This fragment was inserted downstream of the YFP 817 sequence into a Gateway donor vector through enzymatic restriction and ligation 818 (p843). The YFP-LARP6C $\triangle L A M$ fusion construct was transferred into a plant binary 819 vector under the control of the LARP6C native promoter through Gateway cloning between p843 and p788 (p844).

The binary vector pGEX2-GEX2GFP was obtained from (Mori et al., 2014).

822 Binary plasmids for transient assays were prepared as follow. DNA fragments 823 encompassing a basal CaMV35S promoter without enhancer sequences (Töpfer et al., 824 1987), modified MGD2 5'-UTRs, and the YFP CDS were ordered from GeneCust 825 (http://www.genecust.com/fr) and subcloned into a Gateway donor vector. DNA cassettes were then introduced into a plant binary vector containing the HPTII 827 (hygromycin) resistance gene (vector CTL575, a derivative of pCAMBIA-1300). The 828 LARP6C coding sequence was fused downstream of the tagRFP fluorescent reporter 829 and placed under the control of a strong CaMV35S promoter by Gateway 830 recombination with the plant binary entry vector p-SITE-6C1 (Martin et al., 2009).

To prepare a translational MGD2-YFP fusion, we amplified the MGD2 genomic region starting from nucleotide -579 from the ATG to the last nucleotide before the stop codon using primers 31 and 32 (Supplemental Data Set S3). The complete PCR 834 product was cloned into pDONR221 via the TOPOisomerase cloning technique to 835 generate entry vector pDONR221-MGD2no-stop. To generate the plant expression 836 vector, LR Clonase was used to recombine pDONR221-MGD2no-stop into the 837 pB7FWG,0 Gateway binary vector in frame with C-terminal YFP. Primary 838 transformants were selected using BASTA selection.

\section{Pollen phenotyping}


841 Pollen transmission tests were conducted through hand pollination of wild-type pistils with pollen from mutant and complemented homozygous plants. The number of offspring carrying the mutation was scored either by PCR-based genotyping or by selection of antibiotic/herbicide-resistant seedlings. The number of mutant seedlings was expressed as a percentage over the total number of seedlings and transmission efficiency of the mutant gametophyte calculated as $T E=$ ((No of mutant / No of WT) x 100). Experiments were conducted in triplicate. Pollen tube guidance efficiency was scored either by semi in vivo (Palanivelu and Preuss, 2006) or in vivo assays by observing pistils hand pollinated with the mutant pollen of interest and stained with aniline blue (Mori et al., 2006). Emasculated pistils were from ms1 (male sterility 1) heterozygous plants. This guaranteed that the pistils were not contaminated with wild-type pollen, as the ms1-1 mutant is male sterile (van der Veen and Wirtz, 1968; Ito et al., 2007). Monitoring of pollen tube guidance in the presence of galvestine1 was conducted through semi in vivo assays on plates containing $1 \%$ DMSO for mock treatment or 5 or $10 \mu \mathrm{M}$ of galvestine-1 with a final concentration of $1 \%$ DMSO. For all pollen tube guidance assays, in vivo aniline blue staining and semi in vivo as well as in vivo blue dot assays were performed according to (Kulichová et al., 2020).

\section{Pollen and pollen tube collection for total RNA and protein extraction}

Approximately $250 \mu \mathrm{L}$ equivalent of open flowers were collected in $1.5 \mathrm{~mL}$ Eppendorf tubes for each respective genotype. To collect mature pollen, flowers were vigorously and briefly vortexed with $500 \mu$ of RIPA extraction buffer for protein extraction or with pollen germination medium for RNA extraction and spun in a benchtop centrifuge for 1 minute. Media were removed and pellets stored at $-80^{\circ} \mathrm{C}$ until extraction. For pollen activation/germination, the pollen pellet was resuspended in liquid pollen germination medium and incubated for $1 \mathrm{~h}$ at $22^{\circ} \mathrm{C}$. One biological replicate corresponds to one sample of $250 \mu \mathrm{L}$ equivalent of open flowers.

\section{Microscopy}

Subcellular localization experiments in pollen grain and pollen tubes were conducted with an LSM700 confocal microscope (Zeiss) using emission/excitation wavelengths: $405 \mathrm{nM} /$ 420-480 nM for DAPI, $555 \mathrm{nM} /$ 600-700 nM for tRFP and $488 \mathrm{nM} /$ 490-555 $\mathrm{nM}$ for YFP and GFP. Mature pollen grains were collected by dipping open flowers into 
874 a drop of DAPI solution (1X PBS pH 7, 0.5\% Triton-X100, $1 \mu \mathrm{g} / \mathrm{mL} 4$ ',6-diamino-2875 phenylindole) on a glass slide. To collect immature pollen grains at the microspore, bi876 and tricellular stages, anthers were collected from unopened buds, placed in a drop of

877 DAPI solution on a glass slide, and gently crushed to free the pollen grains from pollen 878 sacs. Pollen tubes germinated and grown as described in the Supplemental Methods 879 were directly observed from the germination slide. For cytoskeleton co-localization, 880 PRO LARP6C-tRFP:LARP6C was co-infiltrated in Nicotiana benthamiana leaves with 881 either p35S:GFP-AtTUB6, p35S:GFP-FABD2, or pUBQ-LifeAct:GFP. Confocal 882 images were taken under a Nikon Eclipse Ti confocal microscope equipped with a 883 CSU-X1 spinning disk module and Andor iXon3 EMCCD camera, as well as with a 884 Zeiss LSM880 confocal microscope, and captured with ZEN 2.3v software. Images 885 were analyzed and assembled with ImageJ/ Fiji (http://imagej.net/http://fiji.sc/Fiji), 886 Adobe Photoshop CS6 (www. adobe.com), Ink-scape (www.inkscape.org), and NIS 887 Elements (LIM) software. For YFP quantification in pollen tubes, MGD2-YFP signals 888 in the respective genotypes were captured under a Zeiss LSM880 confocal microscope 889 using an Argon 488 laser and detected with GasP detector with fixed configurations. 890 Zen (blue edition) software was used to quantify pixel intensity, and data were 891 processed in Excel.

892

\section{Pharmacological treatment}

894 For the galvestine-1 inhibition assay, pollen tubes were grown on solid pollen 895 germination medium containing $1 \%$ DMSO for mock treatment or 5 or $10 \mu \mathrm{M}$ of 896 galvestine-1 at a final concentration of 1\% DMSO. For Brefeldin A treatment, 897 Arabidopsis pollen tubes were grown for $1 \mathrm{~h}$ in vitro and stained by incubating with 1 898 HM FM4-64 (Thermo Fisher Scientific, https://www.thermofisher.com) concomitantly 899 with Brefeldin A at $25 \mu \mathrm{M}$ (BFA, Sigma, www.sigmaaldrich.com, BFA stock solution at $90050 \mathrm{mM}$ in DMSO) in liquid pollen germination medium for 5 and $10 \mathrm{~min}$. To visualize 901 lipid droplets, Bodipy stain (505/515) at a final concentration of $2 \mu \mathrm{M}$ and Nile red stain $902(552 / 636)$ at a final concentration of $(0.1 \mu \mathrm{g} / \mathrm{mL})$ were simultaneously added to 903 germinated pollen tubes and incubated for 3 min prior to imaging.

904

\section{Total RNA extraction and RT-qPCR}


906 For RNA-sequencing purpose, total RNA was purified from mature pollen grain as 907 follow. 0.5 to $3 \mathrm{mg}$ of dry pollen was ground with a Silamat S6 mixing device (Ivolcar 908 Vivadent, Schaan, Liechtenstein) in $500 \mu \mathrm{L}$ of GuHCl buffer (8 M GuHCl, 20 mM MES, $90920 \mathrm{mM}$ EDTA, at pH 7, $50 \mathrm{mM} \beta$-mercaptoethanol) and with 5 glass beads. Following 9101 min vortexing at full speed and 10 min incubation on ice, RNAs were separated from 911 proteins via two consecutive phenol-chloroform-IsoAmylAlcool (IAA) (25:24:1) 912 extractions, followed by one extraction with chloroform-IAA (24:1), consisting of 1 min 913 vortexing at full speed and $10 \mathrm{~min}$ centrifugation at $16^{\circ} \mathrm{C}, 14,000 \mathrm{~g}$. RNAs are then 914 precipitated in the presence of $0.7 \mathrm{vol}$ isopropanol, $1 / 20^{\text {th }} \mathrm{CH}_{3} \mathrm{COOH}$ at $1 \mathrm{M}$ for 20 min 915 at $-20^{\circ} \mathrm{C}$. Following 20 min centrifugation at $4^{\circ} \mathrm{C}, 14,000 \mathrm{~g}$, the RNA pellet was washed 916 in $90 \% \mathrm{EtOH}$ and resuspended in RNAse free water. The average efficiency of pollen 917 RNA extraction was 5-6 $\mu \mathrm{g}$ of RNA per mg of dry pollen. To measure MGD2 (YFP918 MGD2 and endogenous MGD2) mRNA levels, total RNA was extracted from $5 \mathrm{mg}$ of 919 dry pollen and $250 \mu \mathrm{l}$ equivalent of flowers used to grow pollen tubes for $4 \mathrm{~h}$ in vitro 920 from (MGD2-YFP; LARP6C) and (MGD2-YFP; larp6c-3) homozygous lines. One 921 biological replicate corresponds to $5 \mathrm{mg}$ of dry pollen and $250 \mu \mathrm{L}$ equivalent flowers for pollen tubes. A total of three biological and six technical replicates were generated. MGD2 native primers and TUB8 were used for measurement and normalization, respectively. Relative expression was computed as above.

To quantify YFP mRNA levels in $N$. benthamiana transient assays, total RNA was isolated from the samples using a Monarch Total RNA Miniprep Kit (New England 927 Biolabs), and genomic DNA was eliminated using gDNA removal columns and oncolumn DNasel-treated (New England Biolabs). $5 \mu \mathrm{g}$ of total RNA was reverse transcribed with a SuperScript IV kit (Life Technology) using an oligodT 18 primer. qPCR was conducted on a LightCycler 480 Multiwell Plates 384-well themocycler (Roche Applied Sciences) with the following amplification program: 5 min $95^{\circ} \mathrm{C}, 40$ cycles of $15 \mathrm{~s}$ at $95^{\circ} \mathrm{C}$, and $1 \mathrm{~min}$ at $60^{\circ} \mathrm{C}$. After amplification, melting curve analysis was performed with a temperature gradient of $0.1^{\circ} \mathrm{C} / \mathrm{s}$ from $60^{\circ} \mathrm{C}$ to $95^{\circ} \mathrm{C}$. The PCR mixture contained Takyon qPCR master mix (Eurogentec), $500 \mathrm{nM}$ gene-specific primers, and $1 \mu \mathrm{L}$ cDNA in a total reaction volume of $10 \mu \mathrm{L}$. Primer efficiencies were determined using standard curves, with 10 -fold serial dilution series ranging from $1 \times 10^{1}$ to $1 \times 10^{6}$ of the cDNA samples. Relative quantification was performed by the $\Delta \Delta \mathrm{C}_{\mathrm{T}}$ method (Livak and Schmittgen, 2001), with YFP and HPTII used as the target and 
939 reference gene, respectively. The sequences of the primers used for RT- and qPCR 940 are shown in Supplemental Table S2.

941

942 RNA-seq, RIP-seq, bioinformatics

943 Techniques related to large scale analyses (RNA-seq and RIP-seq), as well as the

944 bioinformatic analyses, are detailed in the Supplemental Methods.

\section{Protein-related techniques}

947 Total protein extracts were separated by SDS PAGE on an acrylamide gel before being 948 electrotransferred onto a PVDF membrane $(0.45 \mu \mathrm{M}$, Merck Millipore). After saturation 949 in a (1X TBS - $0.1 \%$ tween - $5 \%$ fat-free dry milk) solution, the membranes were 950 incubated with the appropriate primary then secondary antibodies, washed three times 951 with a 1 X TBS - $0.1 \%$ Tween solution, and chemiluminescence revealed with an EMD 952 Millipore Immobilon Western Chemiluminescent HRP Substrate kit. Antibodies against 953 the LARP6C protein were custom made (at Agro-Bio, La Ferté St Aubin, France) 954 through immunization of rabbits with the peptide "KRTSQFTDRDREELGQ" (amino 955 acids 220-235). Anti-LARP6C antibodies are utilized at 1/1000 dilution in a (1X TBS $956 \quad 0.1 \%$ Tween - $5 \%$ fat-free dry milk) solution and incubated overnight at $4^{\circ} \mathrm{C}$ with gentle 957 shaking. Secondary antibodies were anti-rabbit HRP (Bio-Rad) and used at a 1/5,000 958 dilution. Anti-ACTIN antibodies were from Affinity Bio Reagents (Golden, CO, USA) 959 and used at 1/15,000 dilution. Secondary antibodies were anti-mouse HRP (Bio-Rad) 960 used at a 1/10,000 dilution. For transient assays, the YFP protein was detected using 961 the GFP antibody (Clontech) at 1/5,000 and the UGPase antibody (Agrisera) at 1/7,500 962 dilution, both incubated overnight at $4^{\circ} \mathrm{C}$. Secondary antibodies anti-mouse HRP (Bio963 Rad) used at a 1/2,000 dilution and anti-rabbit HRP (Bio-Rad) used at a 1/5,000 dilution 964 were incubated for YFP and UGPase detection, respectively, for $1 \mathrm{~h}$ at room 965 temperature. To detect endogenous MGD2, proteins were extracted from $5 \mathrm{mg}$ of dry 966 pollen and $250 \mu$ l equivalent of flowers used to germinate pollen tubes in vitro for $1 \mathrm{~h}$ 967 or 4h from (MGD2-YFP; LARP6C) and (MGD2-YFP; larp6c-3) homozygous lines. One 968 biological replicate corresponds to $5 \mathrm{mg}$ of pollen grain, and $250 \mu \mathrm{L}$ equivalent open 969 flowers for pollen tubes. A total of three replicates for dry pollen and $1 \mathrm{~h}$ activated pollen, 970 and two replicates for $4 \mathrm{~h}$ pollen tubes were generated. After SDS-PAGE separation, 971 membrane blocking was performed with $5 \%$ milk for $30 \mathrm{~min}$ at room temperature. The 
972 membrane was incubated with antibodies against MGD2 (a gift from Eric Maréchal

973 (LPCV, Grenoble) (Awai et al., 2001), at $1 / 500$ dilution, overnight at $4^{\circ} \mathrm{C}$. Secondary

974 anti-Rabbit HRP (Agrisera) was used at 1/10,000 dilution and incubated for $1 \mathrm{~h}$ at room

975 temperature prior to membrane development.

976 For RNA-protein binding in vitro assays, the LARP6C La-module (amino acids 977 137-223) was recombinantly expressed as in (Merret et al., 2013b). The hexahistidine 978 tag (His-tag) was removed by proteolysis via incubation at $4^{\circ} \mathrm{C}$ overnight with HRV $3 \mathrm{C}$ 979 Protease in a buffer containing $50 \mathrm{mM}$ Tris-HCl pH 7.25, $100 \mathrm{mM} \mathrm{KCl}, 0.2 \mathrm{mM}$ EDTA, $9801 \mathrm{mM}$ DTT, $10 \%$ glycerol. The cleaved His-Tag and the protease were separated from 981 the La-module through retention onto a Ni-NTA column. The protein was then 982 subjected to heparin column purification and dialysis in a final buffer as in (Merret et 983 al., 2013b).

984

\section{ITC and EMSA}

986 RNA oligos were custom made from IBA-Lifescience GmbH (Göttingen, Germany) at 987 a $1 \mu$ Molar scale and HPLC purified. The lyophilized RNAs were resuspended in 988 RNAse free water (DEPC treated) at concentrations ranging from 2.5 to $3 \mathrm{mM}$. The 989 ITC experiments were performed as in (Merret et al., 2013b) on an iTC200 990 microcalorimenter (Malvern). Here, 20 injections of $2 \mu \mathrm{L}$ RNA solutions at 200-320 $\mu \mathrm{M}$ 991 concentration were added to protein solutions (LARP6C La-module) at 20-30 $\mu \mathrm{M}$ with 992 a computer-controlled $40 \mu \mathrm{L}$ syringe. Control titrations of RNA into buffer alone and 993 into protein solution without the His-tag were performed. The heat per injection 994 normalized per mole of injectant versus molecular ratio was analyzed with the 995 MicroCal-Origin 7.0 software package and fitted using a nonlinear least-square 996 minimization algorithm using a theoretical single-site binding model. $\Delta \mathrm{H}$ (reaction 997 enthalpy change in $\mathrm{kcal} / \mathrm{mol}$ ), $\mathrm{K}_{\mathrm{b}}$ (binding constant equal to $1 / \mathrm{Kd}$ ), and $\mathrm{n}$ (molar ratio 998 between the two proteins in the complex) were the fitting parameters. The reaction 999 entropy was calculated using the equations $\Delta G=-R T \ln K b(\mathrm{R}=1.986 \mathrm{cal} / \mathrm{mol} \mathrm{K} ; \mathrm{T}=$ $1000298 \mathrm{~K}$ ) and $\Delta G=\Delta H-T \Delta S$. All ITC experiments were repeated at least three times, 1001 and a detailed analysis of the thermodynamic parameters and errors calculated as the 1002 standard deviation from the mean value are reported in Supplemental Table S1. For 1003 EMSA (Electrophoretic Mobility Shift Assay), RNAs were 5'-labelled with $\gamma$-32P-ATP 1004 using T4 polynucleotide kinase. Recombinant protein and RNA were mixed and 


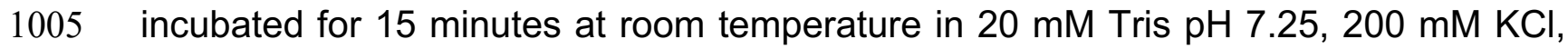
$10065 \%$ of glycerol, $1 \mathrm{mM}$ DTT, $0.1 \mathrm{mg} / \mathrm{mL}$ of BSA and 0.7 units of RNase inhibitors 1007 (RNaseOUT, Invitrogen). Each reaction mixture (22 $\mu \mathrm{L})$ contained $3 \mathrm{nM}$ of labelled 1008 RNA oligo and varying amounts of LARP6C La-module (3-fold serial dilutions from an $100988 \mu \mathrm{M}$ sample). The experiments were performed in the absence or presence of 1010 unlabelled mixed tRNAs from E. coli MRE $600(0.01 \mathrm{mg} / \mathrm{mL})$. After the addition of $2 \mu \mathrm{L}$ 1011 of $30 \%$ Ficoll, the samples were loaded on a $9 \%$ native polyacrylamide gel prerun at $1012100 \mathrm{mV}$ for 1 hour at $4^{\circ} \mathrm{C}$ in $0.5 x$ TBE (Tris-borate-EDTA buffer). A typical EMSA 1013 experiment was run at $4^{\circ} \mathrm{C}$ for 1 hour at $125 \mathrm{mV}$. Gels were dried onto 3MM 1014 chromatography paper, exposed to a phosphoimaging plate overnight, analysed on a 1015 Typhoon Trio phosphoimager, and quantified with Image Quant TL software. The 1016 fraction of bound RNA was plotted versus the protein concentrations. To determine the 1017 dissociation constants, the data were fitted with Origin 8.0 to a modified Hill equation 1018 (Ryder et al., 2008) using non-linear least squares methods and assuming a 1:1 1019 stoichiometry.

\section{Nicotiana benthamiana transient assays}

1022 Plasmids B1-YFP, B3-YFP and RFP-LARP6C were respectively transformed into 1023 Agrobacterium tumefaciens strain LB4404. Transformed bacteria were cultivated until 1024 they reached OD 0.8 and used to infiltrate fully expanded leaves from eight-week-old $1025 N$. benthamina plants using a needless syringe as described in (Ruiz et al., 1998). 1026 When double transformations were performed (B1-YFP and RFP-LARP6C or B3-YFP 1027 and RFP-LARP6C), an equimolar mixture of Agrobacterium cultures (OD 0.4) was 1028 used for transformation. For each transformation, we also used an Agrobacterium 1029 culture carrying a plasmid (OD 0.2) allowing the expression of the P19 suppressor of 1030 RNA silencing (Lasierra and Prat, 2018).Three days post-infiltration (3 dpi), 1031 transformed leaf segments were collected, flash frozen, and total RNA or protein 1032 extracted. Alternatively, at 3 dpi transformed leaves were observed by confocal 1033 microscopy to monitor YFP and tRFP signals.

\section{Accession Numbers}

1036 Sequence data in this study can be found under the following accession numbers: 1037 Bioproject PRJNA557669. RNA-Seq-Col0-Rep 1: SRR9887494; RNA-Seq-Col0-Rep 
1: SRR9887493; RNA-Seq-larp6c-3-Rep 1; SRR9887496; RNA-Seq-larp6c-3-Rep 2 SRR9887495; RIP-Col0-Input-Rep 1: SRR9887498; RIP-Col0-Eluate-Rep 1: SRR9887500; RIP-Col0-Input-Rep 2: SRR9887497; RIP-Col0-Eluate-Rep 2: SRR9887499; RIP-LARP6C-Input-Rep 1: SRR9887490; RIP-LARP6C-Eluate-Rep 1: SRR9887492; RIP-LARP6C-Input-Rep 2: SRR9887489; RIP-LARP6C-Eluate-Rep 2 :

1043 SRR9887491.

\section{Supplemental Data}

1047 Supplemental Figure S1: Expression profiles of LARP6A, 6B and 6C mRNAs across 1048 development.

1049 Supplemental Figure S2: Pollen maturation, germination, and pollen tube growth of 1050 larp6c loss-of function mutants.

1051 Supplemental Figure S3: Schematic representation of LARP6C transgenes and 1052 immunoblotting analyses of their accumulation. Semi in vivo pollen tube guidance competition assays. Analysis of LARP6A function in male fertilization.

1054 Supplemental Figure S4: Pollen attraction competence of larp6c-3 and 6c4 ovules.

1055 Supplemental Figure S5: Confocal analyses of LARP6C-tRFP and/ LARP6A-GFP in 1056 pollen.

1057 Supplemental Figure S6: Reproducibility of the RIP-seq data, RIP-seq filtering 1058 workflow, representation of the position of A and/or B boxes on the 5'-UTRs of LARP6C 1059 targets.

1060 Supplemental Figure S7: ITC and EMSA assessment of LARP6C La-module binding 1061 to A type oligos.

1062 Supplemental Figure S8: Reproducibility of the RNA-seq data.

1063 Supplemental Figure S9: Model of the molecular functions of LARP6C in male 1064 fertilization.

1065 Supplemental Table S1: Thermodynamic parameters of the calorimetric analyses 1066 shown in Figure 4B, 4D and Supplemental Figure 6A.

1067 Supplemental Table S2: List and sequences of primers used for q-PCR, genotyping 1068 and cloning.

1069 Supplemental Methods

1070 Supplemental Data Set S1: Results of RIP-seq experiment.

1071 Supplemental Data Set S2: Results of RNA-Seq experiment. 
1072 Supplemental Movie S1: LARP6C foci move along microtubules.

1073

1074 ACKNOWLEDGEMENTS

1075 This work was supported by the CNRS, the University of Perpignan (UPVD), the Institut 1076 Universitaire de France (IUF) and the Bio-Environnement platform through utilization 1077 of the confocal microscope. This study is set within the framework of the "Laboratoires 1078 d'Excellence (LABEX)" TULIP (ANR-10-LABX-41). EB was the recipient of a PhD grant 1079 from the UPVD, Doctoral School ED305. CGL jr. is the recipient of a short-term contract 1080 supported by the ANR Heat-EpiRNA (ANR-17-CE20-007-01). SH, KK and DH are supported by GACR grant numbers 17-23203S and 18-02448S. SH is also supported

1082 by European Regional Development Fund-Project "Centre for Experimental Plant 1083 Biology" (No. CZ.02.1.01/0.0/0.0/16_019/0000738). ICG was supported by a Royal 1084 Society Newton International fellowship (ref. NF140482). IGC and MRC thank the 1085 Centre for Biomolecular Spectroscopy at King's College London funded by a capital award from the Wellcome Trust. We thank David Twell group (Leicester University) for sharing LAT52:H2B-GFP marker, Martin Potoský (Cell Biology, IEB Prague), Eva Kollárová and Fatima Cvrčková (Faculty of Science, Charles University, Prague) for the cytoskeleton constructs. The authors would like to thank Eric Maréchal (LPCV,

1090 Grenoble) for sharing MGD2 antibodies and galvestine-1, as well as for fruitful discussions on LARP6C targets' functions, in particular those involved in lipid biology. This work was funded by the CNRS, The University of Perpignan (UPVD), the Agence Nationale pour la Recherche (ANR) grant Heat-EpiRNA ( $n^{\circ}$ : ANR-17-CE20007-01); a Bonus Quality Research (BQR) funded by the University of Perpignan ; a Collaborative PICS Project (LARP\&STRESS, $n^{\circ}$ 6170) funded by CNRS; Grantová agentura České republiky (GACR) grants ( $n^{\circ}: 17-23203 S$ and 18-02448S), European

1097 Regional Development Fund-Project "Centre for Experimental Plant Biology" (No. 1098 CZ.02.1.01/0.0/0.0/16_019/0000738) and the Royal Society Newton International 1099 fellowship (ref. NF140482).

\section{AUTHOR CONTRIBUTIONS}

1102 EB and SH performed experiments and analyzed the data. CP helped EB with confocal 1103 microscopy analyses. VJ helped EB with plant maintenance and reciprocal crossed. MCC 1104 performed RNA-seq and RIP-seq bioinformatics. VK and KK assisted SH with in vitro pollen 1105 tube assays and VK assisted SH with experiments presented in Figure 9. ICG and MRC 
1106 designed and analyzed EMSA and ITCs; ICG performed the experiments. CGL Jr. ran 1107 experiments from Figure 8. EM contributed his knowledge on lipid metabolism and suggested 1108 a role for MGD2 as cargo for TAGs. CBA coordinated the work, designed the research, 1109 analyzed data and wrote the paper. JMD helped with data analysis. EB, SH, MRC, JMD and $1110 \mathrm{DH}$ revised the manuscript.

\section{REFERENCES}

1113 Ambrose, J.C. and Cyr, R. (2007). The Kinesin ATK5 Functions in Early Spindle 1114 Assembly in Arabidopsis. Plant Cell 19: 226-236.

1115 Awai, K., Maréchal, E., Block, M.A., Brun, D., Masuda, T., Shimada, H., Takamiya, 1116 K., Ohta, H., and Joyard, J. (2001). Two types of MGDG synthase genes, found 1117 widely in both $16: 3$ and 18:3 plants, differentially mediate galactolipid syntheses in photosynthetic and nonphotosynthetic tissues in Arabidopsis thaliana. Proc. Natl. Acad. Sci. U. S. A. 98: 10960-5.

Belostotsky, D.A. (2003). Unexpected complexity of poly(A)-binding protein gene families in flowering plants: three conserved lineages that are at least 200 million years old and possible auto- and cross-regulation. Genetics 163: 311-9.

Belostotsky, D.A. and Meagher, R.B. (1996). A pollen-, ovule-, and early embryospecific poly $(A)$ binding protein from Arabidopsis complements essential functions in yeast. Plant Cell 8: 1261-75.

Botella, C., Sautron, E., Boudiere, L., Michaud, M., Dubots, E., Yamaryo-Botté, Y., Albrieux, C., Marechal, E., Block, M.A., and Jouhet, J. (2016). ALA10, a Phospholipid Flippase, Controls FAD2/FAD3 Desaturation of Phosphatidylcholine in the ER and Affects Chloroplast Lipid Composition in Arabidopsis thaliana. Plant Physiol. 170: 1300-14.

Botté, C.Y. et al. (2011). Chemical inhibitors of monogalactosyldiacylglycerol synthases in Arabidopsis thaliana. Nat. Chem. Biol. 7: 834-842.

Buchan, J.R. (2014). mRNP granules. Assembly, function, and connections with disease. RNA Biol. 11: 1019-30. 
1140 Bullock, S.L. (2011). Messengers, motors and mysteries: sorting of eukaryotic mRNAs by cytoskeletal transport. Biochem. Soc. Trans. 39: 1161-5.

1142 Cai, L., Fritz, D., Stefanovic, L., and Stefanovic, B. (2010a). Binding of LARP6 to 1143 the conserved 5' stem-loop regulates translation of mRNAs encoding type I 1144 collagen. J. Mol. Biol. 395: 309-26.

1145 Cai, L., Fritz, D., Stefanovic, L., and Stefanovic, B. (2010b). Nonmuscle myosindependent synthesis of type I collagen. J. Mol. Biol. 401: 564-78.

Challa, A.A. and Stefanovic, B. (2011). A novel role of vimentin filaments: binding and stabilization of collagen mRNAs. Mol. Cell. Biol. 31: 3773-89.

Clough, S.J. and Bent, A.F. (1998). Floral dip: a simplified method for Agrobacteriummediated transformation of Arabidopsis thaliana. Plant J. 16: 735-43.

Cvrčková, F. and Oulehlová, D. (2017). A new kymogram-based method reveals unexpected effects of marker protein expression and spatial anisotropy of cytoskeletal dynamics in plant cell cortex. Plant Methods 13: 19.

Dermit, M., Dodel, M., Lee, F.C.Y., Azman, M.S., Schwenzer, H., Jones, J.L., Blagden, S.P., Ule, J., and Mardakheh, F.K. (2020). Subcellular mRNA Localization Regulates Ribosome Biogenesis in Migrating Cells. Dev. Cell 55: 298-313.e10.

Eliscovich, C. and Singer, R.H. (2017). RNP transport in cell biology: the long and winding road. Curr. Opin. Cell Biol. 45: 38-46.

Feng, Q.-N., Liang, X., Li, S., and Zhang, Y. (2018). The ADAPTOR PROTEIN-3 Complex Mediates Pollen Tube Growth by Coordinating Vacuolar Targeting and Organization. Plant Physiol. 177: 216-225.

Grebnev, G., Ntefidou, M., and Kost, B. (2017). Secretion and Endocytosis in Pollen Tubes: Models of Tip Growth in the Spot Light. Front. Plant Sci. 8: 154.

Hafidh, S., Potěšil, D., Fíla, J., Feciková, J., Čapková, V., Zdráhal, Z., and Honys, D. (2014). In search of ligands and receptors of the pollen tube: the missing link in pollen tube perception. Biochem. Soc. Trans. 42: 388-94.

Hafidh, S., Potěšil, D., Müller, K., Fíla, J., Michailidis, C., Herrmannová, A., Feciková, J., Ischebeck, T., Valášek, L.S., Zdráhal, Z., and Honys, D. (2018). Dynamics of the Pollen Sequestrome Defined by Subcellular Coupled Omics. Plant Physiol. 178: 258-282.

Hajdukiewicz, P., Svab, Z., and Maliga, P. (1994). The small, versatile pPZP family of Agrobacterium binary vectors for plant transformation. Plant Mol. Biol. 25: 989_ 
94.

1175

1176

1177

1178

1179

1180

1181

1182

1183

1184

1185

1186

1187

1188

1189

1190

1191

1192

1193

1194

1195

1196

1197

1198

1199

1200

1201

1202

1203

1204

1205

1206

1207

Hau, H.T.A. et al. (2020). Maternal Larp6 controls oocyte development, chorion formation and elevation. Development 147.

Hellman, L.M. and Fried, M.G. (2007). Electrophoretic mobility shift assay (EMSA) for detecting protein-nucleic acid interactions. Nat. Protoc. 2: 1849-1861.

Higashiyama, T. and Takeuchi, H. (2015). The mechanism and key molecules involved in pollen tube guidance. Annu. Rev. Plant Biol. 66: 393-413.

Honys, D., Combe, J.P., Twell, D., and Capková, V. (2000). The translationally repressed pollen-specific ntp303 mRNA is stored in non-polysomal mRNPs during pollen maturation. Sex. Plant Reprod. 13: 135-144.

Honys, D. and Twell, D. (2004). Transcriptome analysis of haploid male gametophyte development in Arabidopsis. Genome Biol. 5: R85.

Hubstenberger, A. et al. (2017). P-Body Purification Reveals the Condensation of Repressed mRNA Regulons. Mol. Cell 68: 144-157.e5.

Ischebeck, T. (2016). Lipids in pollen - They are different. Biochim. Biophys. Acta 1861: 1315-1328.

Ito, T., Nagata, N., Yoshiba, Y., Ohme-Takagi, M., Ma, H., and Shinozaki, K. (2007). Arabidopsis MALE STERILITY1 Encodes a PHD-Type Transcription Factor and Regulates Pollen and Tapetum Development. Plant Cell 19: 3549-3562.

Johnson, M.A. and Preuss, D. (2002). Plotting a course: multiple signals guide pollen tubes to their targets. Dev. Cell 2: 273-81.

Kelly, A.A., van Erp, H., Quettier, A.-L., Shaw, E., Menard, G., Kurup, S., and Eastmond, P.J. (2013). The SUGAR-DEPENDENT1 Lipase Limits Triacylglycerol Accumulation in Vegetative Tissues of Arabidopsis. PLANT Physiol. 162: 1282-1289.

Khan, B.R., Adham, A.R., and Zolman, B.K. (2012). Peroxisomal Acyl-CoA oxidase 4 activity differs between Arabidopsis accessions. Plant Mol. Biol. 78: 45-58.

Klepikova, A. V, Logacheva, M.D., Dmitriev, S.E., and Penin, A.A. (2015). RNAseq analysis of an apical meristem time series reveals a critical point in Arabidopsis thaliana flower initiation. BMC Genomics 16: 466.

Kulichová, K., Kumar, V., Steinbachová, L., Klodová, B., Timofejeva, L., Juříček, M., Honys, D., and Hafidh, S.S. (2020). PRP8A and PRP8B spliceosome subunits act coordinately to control pollen tube attraction in Arabidopsis thaliana. Development 147. 
Lasierra, P. and Prat, S. (2018). Transient Transactivation Studies in Nicotiana benthamiana Leaves. Methods Mol. Biol. 1794: 311-322.

1210 Li, S., van Os, G.M.A., Ren, S., Yu, D., Ketelaar, T., Emons, A.M.C., and Liu, C.-M.

1211 (2010). Expression and functional analyses of EXO70 genes in Arabidopsis 1212 implicate their roles in regulating cell type-specific exocytosis. Plant Physiol. 154: 1213 1819-30.

1214 Livak, K.J. and Schmittgen, T.D. (2001). Analysis of Relative Gene Expression Data Using Real-Time Quantitative PCR and the 2- $\Delta \Delta C T$ Method. Methods 25: 402408.

Martin, K., Kopperud, K., Chakrabarty, R., Banerjee, R., Brooks, R., and Goodin,

Manojlovic, Z., Earwood, R., Kato, A., Perez, D., Cabrera, O.A., Didier, R., Megraw, T.L., Stefanovic, B., and Kato, Y. (2017). La-related protein 6 controls ciliated cell differentiation. Cilia 6: 4.

Maraia, R.J., Mattijssen, S., Cruz-Gallardo, I., and Conte, M.R. (2017). The La and related RNA-binding proteins (LARPs): structures, functions, and evolving perspectives. Wiley Interdiscip. Rev. RNA 8.

M.M. (2009). Transient expression in Nicotiana benthamiana fluorescent marker lines provides enhanced definition of protein localization, movement and interactions in planta. Plant J. 59: 150-62.

Martino, L. et al. (2015). Synergic interplay of the La motif, RRM1 and the interdomain linker of LARP6 in the recognition of collagen mRNA expands the RNA binding repertoire of the La module. Nucleic Acids Res. 43: 645-60.

McCue, A.D., Cresti, M., Feijó, J.A., and Slotkin, R.K. (2011). Cytoplasmic connection of sperm cells to the pollen vegetative cell nucleus: potential roles of the male germ unit revisited. J. Exp. Bot. 62: 1621-31.

McElver, J. et al. (2001). Insertional mutagenesis of genes required for seed development in Arabidopsis thaliana. Genetics 159: 1751-63.

Merret, R., Descombin, J., Juan, Y.-T., Favory, J.-J., Carpentier, M.-C., Chaparro, C., Charng, Y.-Y., Deragon, J.-M., and Bousquet-Antonelli, C. (2013a). XRN4 and LARP1 are required for a heat-triggered mRNA decay pathway involved in plant acclimation and survival during thermal stress. Cell Rep. $\mathbf{5}$.

Merret, R., Martino, L., Bousquet-Antonelli, C., Fneich, S., Descombin, J., Billey, É., Conte, M.R., and Deragon, J.-M. (2013b). The association of a La module with the PABP-interacting motif PAM2 is a recurrent evolutionary process that led 
to the neofunctionalization of la-related proteins. RNA 19.

Mochizuki, S., Harada, A., Inada, S., Sugimoto-Shirasu, K., Stacey, N., Wada, T., Ishiguro, S., Okada, K., and Sakai, T. (2005). The Arabidopsis WAVY GROWTH 2 protein modulates root bending in response to environmental stimuli. Plant Cell 17: 537-47.

Mori, T., Igawa, T., Tamiya, G., Miyagishima, S.-Y., and Berger, F. (2014). Gamete attachment requires GEX2 for successful fertilization in Arabidopsis. Curr. Biol.

Mori, T., Kuroiwa, H., Higashiyama, T., and Kuroiwa, T. (2006). GENERATIVE 24: 170-175. CELL SPECIFIC 1 is essential for angiosperm fertilization. Nat. Cell Biol. 8: 6471.

Palanivelu, R. and Preuss, D. (2006). Distinct short-range ovule signals attract or repel Arabidopsis thaliana pollen tubes in vitro. BMC Plant Biol. 6: 7.

Pleskot, R., Pejchar, P., Bezvoda, R., Lichtscheidl, I.K., Wolters-Arts, M., Marc, J., Zárský, V., and Potocký, M. (2012). Turnover of Phosphatidic Acid through Distinct Signaling Pathways Affects Multiple Aspects of Pollen Tube Growth in Tobacco. Front. Plant Sci. 3: 54.

Qin, Y., Leydon, A.R., Manziello, A., Pandey, R., Mount, D., Denic, S., Vasic, B., Johnson, M.A., and Palanivelu, R. (2009). Penetration of the stigma and style elicits a novel transcriptome in pollen tubes, pointing to genes critical for growth in a pistil. PLoS Genet. 5: e1000621.

Reyes, F.C., Buono, R.A., Roschzttardtz, H., Di Rubbo, S., Yeun, L.H., Russinova, E., and Otegui, M.S. (2014). A novel endosomal sorting complex required for transport (ESCRT) component in Arabidopsis thaliana controls cell expansion and development. J. Biol. Chem. 289: 4980-8.

Ruiz, M.T., Voinnet, O., and Baulcombe, D.C. (1998). Initiation and Maintenance of Virus-Induced Gene Silencing. Plant Cell 10: 937-946.

Ryder, S.P., Recht, M.I., and Williamson, J.R. (2008). Quantitative analysis of protein-RNA interactions by gel mobility shift. Methods Mol. Biol. 488: 99-115.

Samaj, J., Müller, J., Beck, M., Böhm, N., and Menzel, D. (2006). Vesicular trafficking, cytoskeleton and signalling in root hairs and pollen tubes. Trends Plant Sci. 11: 594-600.

Scarpin, M.R., Sigaut, L., Temprana, S.G., Boccaccio, G.L., Pietrasanta, L.I., and Muschietti, J.P. (2017). Two Arabidopsis late pollen transcripts are detected in 
cytoplasmic granules. Plant direct 1: e00012.

Schulz-Raffelt, M., Chochois, V., Auroy, P., Cuiné, S., Billon, E., Dauvillée, D., LiBeisson, Y., and Peltier, G. (2016). Hyper-accumulation of starch and oil in a Chlamydomonas mutant affected in a plant-specific DYRK kinase. Biotechnol. Biofuels 9: 55.

Stålberg, K., Ståhl, U., Stymne, S., and OhIrogge, J. (2009). Characterization of two Arabidopsis thaliana acyltransferases with preference for lysophosphatidylethanolamine. BMC Plant Biol. 9: 60.

Synek, L., Schlager, N., Eliáš, M., Quentin, M., Hauser, M.-T., and Žárský, V. (2006). AtEXO70A1, a member of a family of putative exocyst subunits specifically expanded in land plants, is important for polar growth and plant development. Plant J. 48: 54-72.

Synek, L., Vukašinović, N., Kulich, I., Hála, M., Aldorfová, K., Fendrych, M., and Žárský, V. (2017). EXO70C2 Is a Key Regulatory Factor for Optimal Tip Growth of Pollen. Plant Physiol. 174: 223-240.

Takemoto, K., Ebine, K., Askani, J.C., Krüger, F., Gonzalez, Z.A., Ito, E., Goh, T., Schumacher, K., Nakano, A., and Ueda, T. (2018). Distinct sets of tethering complexes, SNARE complexes, and Rab GTPases mediate membrane fusion at the vacuole in Arabidopsis. Proc. Natl. Acad. Sci. U. S. A. 115: E2457-E2466.

Tian, L., Chou, H.-L., Fukuda, M., Kumamaru, T., and Okita, T.W. (2020). mRNA Localization in Plant Cells. Plant Physiol. 182: 97-109.

Töpfer, R., Matzeit, V., Gronenborn, B., Schell, J., and Steinbiss, H.H. (1987). A set of plant expression vectors for transcriptional and translational fusions. Nucleic Acids Res. 15: 5890.

Tse, Y.C., Lo, S.W., Hillmer, S., Dupree, P., and Jiang, L. (2006). Dynamic response of prevacuolar compartments to brefeldin a in plant cells. Plant Physiol. 142: 1442-59.

Twell, D., Wing, R., Yamaguchi, J., and McCormick, S. (1989). Isolation and expression of an anther-specific gene from tomato. Mol. Gen. Genet. 217: 240-5.

van der Veen, J.H. and Wirtz, P. (1968). EMS-induced genic male sterility in Arabidopsis thaliana: A model selection experiment. Euphytica 17: 371-377.

Voigt, B., Timmers, A.C.J., Samaj, J., Müller, J., Baluska, F., and Menzel, D. (2005). GFP-FABD2 fusion construct allows in vivo visualization of the dynamic actin cytoskeleton in all cells of Arabidopsis seedlings. Eur. J. Cell Biol. 84: 595- 
608.

Vukašinović, N. and Žárský, V. (2016). Tethering Complexes in the Arabidopsis Endomembrane System. Front. cell Dev. Biol. 4: 46.

1313 Vukmirovic, M., Manojlovic, Z., and Stefanovic, B. (2013). Serine-threonine kinase receptor-associated protein (STRAP) regulates translation of type I collagen mRNAs. Mol. Cell. Biol. 33: 3893-906.

Weber, C., Nover, L., and Fauth, M. (2008). Plant stress granules and mRNA processing bodies are distinct from heat stress granules. Plant J. 56: 517-30.

Weng, H., Kim, C., Valavanis, C., Wang, Z., and Schwartz, L.M. (2009). Acheron, an novel LA antigen family member, binds to CASK and forms a complex with Id transcription factors. Cell. Mol. Biol. Lett. 14: 273-87.

Woody, S.T., Austin-Phillips, S., Amasino, R.M., and Krysan, P.J. (2007). The WiscDsLox T-DNA collection: an arabidopsis community resource generated by using an improved high-throughput T-DNA sequencing pipeline. J. Plant Res. 120: 157-65.

Ylstra and McCormick (1999). Analysis of mRNA stabilities during pollen development and in BY2 cells. Plant J. 20: 101-8.

Yu, J., Qiu, H., Liu, X., Wang, M., Gao, Y., Chory, J., and Tao, Y. (2015). Characterization of tub4(P287L) , a $\beta$-tubulin mutant, revealed new aspects of microtubule regulation in shade. J. Integr. Plant Biol. 57: 757-69.

Zhang, M., Fan, J., Taylor, D.C., and Ohlrogge, J.B. (2009). DGAT1 and PDAT1 Acyltransferases Have Overlapping Functions in Arabidopsis Triacylglycerol Biosynthesis and Are Essential for Normal Pollen and Seed Development. Plant Cell 21: 3885-3901.

Zhang, Y. and Stefanovic, B. (2016). LARP6 Meets Collagen mRNA: Specific Regulation of Type I Collagen Expression. Int. J. Mol. Sci. 17: 419.

Zheng, Y., Deng, X., Qu, A., Zhang, M., Tao, Y., Yang, L., Liu, Y., Xu, J., and Zhang, S. (2018). Regulation of pollen lipid body biogenesis by MAP kinases and downstream WRKY transcription factors in Arabidopsis. PLoS Genet. 14: e1007880.

Zhou, J.-J., Liang, Y., Niu, Q.-K., Chen, L.-Q., Zhang, X.-Q., and Ye, D. (2013). The Arabidopsis general transcription factor TFIIB1 (AtTFIIB1) is required for pollen tube growth and endosperm development. J. Exp. Bot. 64: 2205-18. 
1346 Figure 1: LARP6C is required for pollen tube guidance. (A), Immunoblot analysis

1347 of steady-state LARP6C protein accumulation. The same blot was probed with 1348 antibodies against LARP6C or ACTIN as a loading control. (B), larp6c-3 and larp6c-4 1349 alleles are loss-of-function mutants. Top panel: schematic representation of the 1350 LARP6C gene and LARP6C protein. Plain boxes represent exons and lines represent 1351 introns. The colour code for exons is identical to that for the conserved protein 1352 domains. The insertion sites of the T-DNAs in larp $6 c-3$ and $6 c-4$ mutants are reported. 1353 Bottom panel: immunoblot analysis of LARP6C accumulation in flowers from wild-type, 1354 larp6c-3 and 6c-4 lines. ACTIN was used as a loading control. (C), Transmission 1355 efficiencies of larp6c-3 and 6c-4 mutant alleles. Transmission Efficiency (TE) was 1356 calculated as: (([No of mutant] / [No of wild type]) x 100). ns: the number of mutant 1357 seedlings in the progeny is not significantly different from the expected number of 1358 mutant seedlings. (D) Scoring of germination rate, pollen tube bursting, and elongation 1359 in vitro and in vivo. Left panels show representative images of in vivo (upper part) and 1360 in vitro (lower part) experiments. On the right, graphs report the frequency of 1361 ungerminated pollen (1), PT emergence (2), PT burst (3), and PT longer than three 1362 lengths of the pollen grain (PT>3) 15 or 30 minutes after pollination. $n$ represents the 1363 number of scored pollen grains. (E), Semi in vivo (SIV) (upper panel) and in vivo 1364 (bottom panel) pollen tube guidance assays. For SIV assays, wild-type ovules were arranged around homozygous $m s 1$ pistil explants pollinated with pollen from various homozygous backgrounds. For in vivo assays, homozygous $m s 1$ pistils were pollinated in planta with pollen from various homozygous genotypes. For each type of

1368 experiment, the numbers of targeted (blue boxes) and non-targeted (white boxes) 1369 ovules were scored and results represented as whisker notched boxplots. The 1370 genotypes of the pollen used for fertilization are recorded below the graphs and $n$ is 1371 the total number of ovules scored in each experiment. The dotted lines respectively 1372 show the wild type mean values of targeted (blue) and non-targeted (dark red) ovules 1373 by wild-type pollen. (F) In vivo scoring of pollen tube behaviours. Wild-type pistils were 1374 pollinated in planta with pollen from various homozygous genotypes (recorded below 1375 the graph) and the behaviour of pollen tubes monitored and scored over a time course: 1376 4h, 8h and 24h after pollination (HAP). Type-I (blue bars): targeting and reception are 1377 normal, Type-Il (white bars): attraction (targeting) is defective. Below the graph are 
1378 representative images of the type of behaviours observed. Scale bars correspond to

$137910 \mu \mathrm{m}$. The table below the graphs report the numbers of scored ovules (n). p-values

1380 were calculated with an unpaired Student t-test. ns: not significant, *: p-value $\leq 0.05,{ }^{* *}$ :

$1381 p$-value $\leq 0.01,{ }^{* *}$ : $p$-value $\leq 0.001$.

1382

1383 Figure 2: Subcellular distribution of LARP6C across pollen development and 1384 pollen tubes. (A) Subcellular localization of fluorescently tagged YFP-LARP6C by 1385 confocal microscopy at different stages of pollen maturation: UNM: uninucleate 1386 microspore, BCP: bicellular pollen, TCP: tricellular pollen, MPG: mature pollen grain 1387 and Pollen tube. Scale bars correspond to $5 \mu \mathrm{m}$ for pollen and $10 \mu \mathrm{m}$ for pollen tube images. Representative images of LARP6C fused at its C-terminus with a tagRFP reporter are shown in Supplemental Figure S5A. (B) Confocal observation of mature pollen grains that stably co-express the LARP6C-tRFP fusion and the H2B-GFP fusion, the GFP-GEX2 fusion, the YFP-DCP1 fusion or the PAB5-GFP fusion. Except for the H2B-GFP marker, which is expressed from the tomato LAT52 promoter, all marker genes are expressed from their own upstream genomic sequences. Scale bars correspond to $5 \mu \mathrm{m}$. In (A) and (B), arrows indicate the position of the Nucleolus (No), Generative Cell Nucleus (GCN), Vegetative Nucleus (VCN), Sperm Cell Nucleus (SCN) and Cytoplasmic Connection (CC). (C) Confocal observation of N. benthamiana

1397 epidermis cells transiently expressing the LARP6C-tRFP fusion co-expressed with 1398 microtubule-binding GFP-AtTUB6 and two actin-binding GFP-FABD2 or LifeAct-GFP reporters. $n=20$ cells from three independent infiltration repeats.

Figure 3: RIP-Seq identification of LARP6C mRNA targets. (A) Volcano plot repartition of the log2 of fold changes between values in the eluate fractions of LARP6C-FH and wildtype for mRNAs found in the LARP6C-FH eluate fraction, according to their p-values obtained through the DESeq2 pipeline. Red dots represent mRNAs that are significantly more represented in LARP6C-FH eluates. A p-value $\leq 0.01$ and $F C \geq 1$ was used as significance criteria. (B) Plotting the RE (Eluate$\mathrm{RPM}$ /Input-RPM) values in LARP6C-FH against the RE values in wild type for the 635 mRNAs highlighted in graph A. mRNAs with a RE6c-FH $\geq 3 R E-W T$ are labelled as blue dots. See Supplemental Figure S6C for a detailed description of the RIP-seq 
1410 pipeline. (C) Pie chart representation of the number of LARP6C targets within the 1411 various functional categories identified.

1412

1413 Figure 4: Loss of LARP6C function affects lipid homeostasis in pollen tubes.

1414 Wild-type and larp6c-3 pollen grains were stained with Bodipy 505/515 and Nile red at 1415 the Mature Pollen Stage (MPG) (A) or in pollen tubes (PTs) (B, C). In (B) PTs were 1416 germinated and grown in vitro. PTs were collected at $2 \mathrm{~h}$ and $4 \mathrm{~h}$ after activation for 1417 staining. Representative images are shown. In (C) PTs grown semi in vivo were 1418 collected $7 \mathrm{~h}$ following pollination (4 hap, pistils are cut at shoulder, placed on plate with 1419 ovules and further incubated 3 hours before scoring). $\mathrm{n}$ represents the number of 1420 pollen tubes with LDs distributed as on the presented image over the total number of 1421 observed pollen grains. Scale bars correspond to $10 \mu \mathrm{m}$ in (A) and to $5 \mu \mathrm{m}$ in (B, C).

1422 (D), Monitoring of pollen tube growth in the presence of galvestine-1. Whisker boxplot 1423 representation of the length of wild-type or larp6c-3 pollen tubes grown in vitro for 4 1424 hours in the presence or absence of galvestine-1. 1: untreated, 2: mock (1\% DMSO), 14253 and 4: $5 \mu \mathrm{M}$ and $10 \mu \mathrm{M}$ galvestine-1. (E) Semi in vivo pollen tube guidance assays. 1426 Assays were conducted either in the presence of 1\% DMSO (untreated) or in the 1427 presence of $10 \mu \mathrm{M}$ of galvestine-1. Results are represented as whisker boxplots. p1428 values were obtained through an unpaired Student t-test, ns: not significant, ${ }^{* * *}$ : $\mathrm{p}$ 1429 value $\leq 0.001$

1431 Figure 5: Pollen tubes deprived of LARP6C are hypersensitive to Brefeldin A 1432 (BFA). Pollen grains were germinated in vitro and after $1 \mathrm{~h}$ activation on germination 1433 medium, pollen tubes were incubated with mock (DMSO) or $25 \mu \mathrm{M}$ BFA. (A) 1434 Representative images of FM4-64 staining captured at the respective times or wild 1435 type (WT) and larp6c-3 PTs treated with mock or BFA. Sketched outline of the PTs 1436 with red dots (counts) describing the appearance of the particle frequency and size, as 1437 detected by the ImageJ particle analysis plug in. (B) Following FM 4-64 staining, the 1438 number (top panel) and size (bottom panel) of BFA bodies was scored over a time 1439 course and represented as violin plots. Number of PTs observed to score particle 1440 number: WT: mock: 23, 10 min: 14, 17 min: 30; larp6c-3: mock: 32, 10 min: 35, 17 min: 1441 25. Number of PTs observed to score particle size: WT: mock: 59, $10 \mathrm{~min}: 62,17 \mathrm{~min}$ : 
95; larp6c-3: mock: 61, $10 \mathrm{~min}: 115,17 \mathrm{~min}: 189$. p-values were obtained with an unpaired Student t-test, ns: not significant, ${ }^{* *}$ : p-value $\leq 0.01,{ }^{* * *}:$-value $\leq 0.001$.

Figure 6: The LARP6C La-module binds to B-type RNA boxes. (A), Consensus sequences of the conserved motifs within the 5'-UTRs of LARP6C-bound mRNAs. Calorimetric (B, D) and EMSA (C, E) analyses of the interaction between the LARP6C La-module (encompassing residues 137-332) and oligos B1, B2 (B, C), $\mathrm{U}_{20}$ (C), B3 and B4 (D, E). In (B) and (D): for each graph, the upper panel corresponds to the raw titration data showing the thermal effect of injecting an RNA oligo solution into a calorimetric cell containing the recombinant LARP6C La-module. The lower panels show the normalized heat per injection values obtained by integrating the raw data and subtracting the heat value of the RNA dilution. The red lines in the graphs for oligos B1 and B2 (B) represent the best fit derived by a non-linear best-square procedure based on an independent binding site model. The dissociation constants $(\mathrm{Kd})$ are indicated for the B1 and B2 oligos; the thermodynamic parameters are shown in Supplemental Table S1. (C, E), EMSAs of LARP6C La-module binding to: B1, B2 or oligo $\mathrm{U}_{20}(\mathrm{C})$, B3 or B4 (E). Decreasing concentrations $(\mu \mathrm{M})$ (88 (lane 1), 29.3 (lane 2), 9.8 (lane 3), 3.3 (lane 4), 1.1 (lane 5), 0.4 (lane 6), 0.12 (lane 7), 0.04 (lane 8) and 0 (lane 9)) of the recombinant LARP6C La-module were mixed with 3nM of 5'-labelled oligos. B stands for Bound, F for Free, red asterisks mark the RNA-protein complex, and the red arrows on panels $E$ shows samples retained into the gel wells. Experiments were conducted in the absence (-tRNA) or presence (+tRNA) of unlabelled competitor

1464 (tRNAmix of E. coli MRE 600 at $0.01 \mathrm{mg} / \mathrm{mL}$ concentration). Graphs in panel C show the quantification of the bound RNA fraction versus the protein concentration in the absence (black lines) or presence (red lines) of tRNA competitor. The values of the dissociation constants are reported. Kd values reported for the EMSA experiments were calculated out of three independent replicates with the following standard deviations: LARP6C-B1 (-tRNA): 0.8 +/- 0.1; LARP6C-B1 (+tRNA): 3.5 +/- 0.3; LARP6C-B2 (-tRNA): 1.9 +/- 0.3; LARP6C-B2 (+tRNA): 5.6 +/- 0.2.

Figure 7: larp6c loss-of-function does not affect the steady-state transcript levels of its target in dry pollen. (A), Heat map representation of the $\log _{2}(\mathrm{RPKM})$ values of genes that are differentially expressed between wild type (WT) and larp6c-3 plants. The heat map was built from the list of DE genes with log2(RPKM) values 
1476 between -2 and +10 (2142 genes: $98.5 \%$ of the DE genes). (B), Plot representation of

1477 the log2 values of the ratios: (larp6c-3/wild type) (upper panel) and log2(6C-FH/wild

1478 type) (lower panel) for the 2174 genes found to be differentially expressed in larp6c-3

1479 mutant pollen. Red lines mark the cut off value (log2(1.5) and $\log 2(1 / 1.5))$. (C) Venn

1480 diagram representation of the number of transcripts that are DE in larp6c-3 and/or

1481 immunoprecipitated by $6 \mathrm{C}-\mathrm{FH}$. Note that of the 115 RIP targets, 19 were not present

1482 in the transcriptomic data from RNA-seq. (D) plot representation of the $\log 2$ (larp6c-

1483 3/WT) for mRNAs identified by RIP-seq. Red lines mark the cut off value $(\log 2(1.5)$

1484 and $\log 2(1 / 1.5))$.

1485

1486 Figure 8: LARP6C binding at the 5'UTR of a reporter construct reduces protein

1487 and increases mRNA levels. (A) Schematic representation of the YFP reporter

1488 constructs, (B) RT-qPCR monitoring of YFP mRNA levels. To normalize YFP mRNA

1489 levels to transformation efficiency, we used the levels of HPTII mRNA encoded by the

1490 HPTII gene carried by the YFP binary plasmid but not the tRFP-LARP6C one. SDs

1491 were calculated from three biological replicates. p-value were obtained using a

1492 Student-t test. ${ }^{* *} \mathrm{P}<0.005$, ns: not significant. (C) Immunoblot analysis of tRFP-

1493 LARP6C and YFP protein levels. Two immunoblots were prepared and respectively

1494 hybridized with anti-LARP6C or GFP antibodies. Levels of UGPase were used as a

1495 loading control. Representative images of three replicates are shown. (D) Confocal

1496 imaging of YFP from leaves not transformed with tRFP-LARP6C (left panels) and of

1497 YFP and tRFP-LARP6C distribution (right panels). Scale bars represent $20 \mu \mathrm{m}$.

1498 Leaves that were observed are different from those used to prepare total RNA and

1499 protein extracts. Representative images of three biological replicates are shown.

1501 Figure 9: LARP6C is involved in the dynamic fine-tuning of MGD2 protein 1502 accumulation in vivo. Monitoring of the levels of MGD2-YFP fusion protein expressed 1503 from the MGD2 native promoter (PROMGD2:MGD2-YFP) and endogenous MGD2 in 1504 wild type (WT) and larp6c-3 plants. (A) Representative images of YFP signal in 1505 heterozygous mature pollen grains at activation: 0, 15 and 30 min after incubation on 1506 in vitro germination medium. Bottom panels show images in pseudo colour with the 1507 associated scale bar representing the intensity of the YFP signal. White arrows and 1508 asterisks respectively point to segregation pollen grains expressing or not expressing 1509 the translational fusion. (B) Live cell imaging of MGD2-YFP distribution in pollen tubes. 
1510 The numbers of pollen tubes with gradient MGD2 distribution toward the tip, uniform 1511 (lost gradient), or no expression of MGD2-YFP are reported in the table below. Scale 1512 bars correspond to $10 \mu \mathrm{m}$. (C) Left panel: fluorescence quantification of MGD2-YFP in

1513 wild type (WT) and larp6c-3 (6c-3) pollen and during the progamic phase (1 and 4h). $1514 \mathrm{n}=3$ biological replicates at each time point, a Student t-test for two independent 1515 samples/two-tailed test was conducted, with ns: not significant, ${ }^{* * * *} p$-value $\leq 0.0001$. 1516 Right panel: immunoblot analysis of endogenous levels of MGD2 in pollen and during 1517 progamic phase in wild type and larp6c-3. Experiments were conducted in two or three 1518 independent replicates labelled R1, R2, and R3. (D) RT-qPCR analysis of MGD2 1519 mRNA levels originating from the MGD2-YFP transgene and endogenous MGD2 gene 1520 in dry pollen and $4 \mathrm{~h}$ pollen tubes. Experiments were conducted in three independent 1521 replicates, and TUBULIN8 mRNA was used as a control. An unpaired Student t-test 1522 was conducted, with n.s.: not significant. Both wild type and larp6c-3 express MGD21523 YFP from the same primary transformant (line 6). In A and B, the MGD2-YFP 1524 transgene is in the heterozygous state and the larp6c-3 allele is homozygous. In $\mathrm{C}$ and 1525 D, wild type and larp6c-3 carry the MGD2-YFP transgene in the homozygous state. 

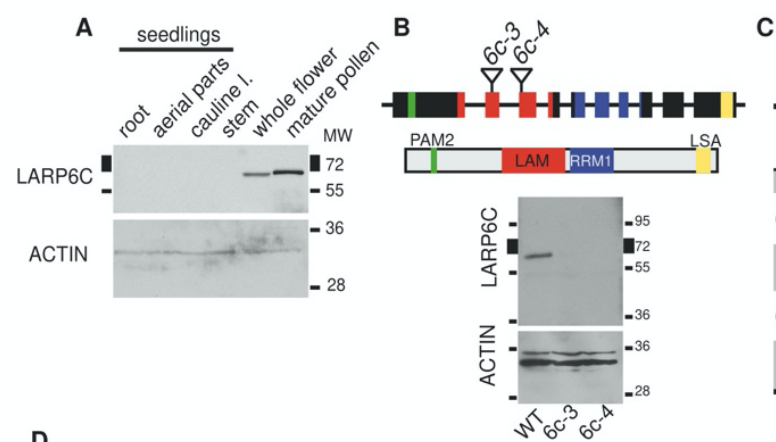

D
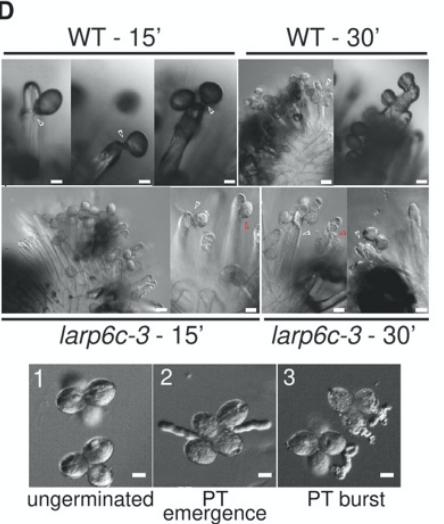

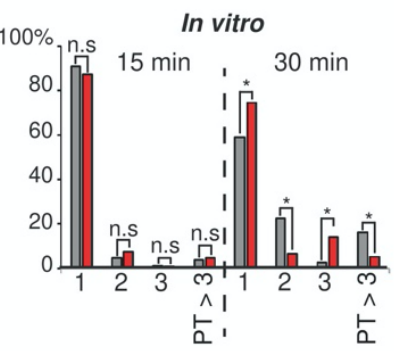

: larp6c-3, $\mathrm{n}=269$

WT, $n=490$

\begin{tabular}{cccccc}
\hline $\begin{array}{c}\text { Parental } \\
\text { Genotypes }\end{array}$ & $\begin{array}{c}\text { Progeny } \\
\text { (total no) }\end{array}$ & $\begin{array}{c}\text { Expected } \\
\text { mutants }\end{array}$ & $\begin{array}{c}\text { Observed } \\
\text { mutants }\end{array}$ & $\begin{array}{c}\text { TE } \\
(\%)\end{array}$ & $\begin{array}{c}\text { khi2; } \\
\text { p-value }\end{array}$ \\
\hline Male Female & & & & \\
6c-3/+ WT & 827 & 413.5 & 352 & 74.1 & $\begin{array}{c}9.14 ; \\
<0.005\end{array}$ \\
WT 6c-3/+ 641 & 320.5 & 321 & 100 & $\begin{array}{c}8.4 ; \\
\text { ns }\end{array}$ \\
6c-4/+ WT 1051 & 525.5 & 426 & 68.2 & $\begin{array}{c}18.8 ; \\
<0.001 \\
0.15 ; \\
\text { ns }\end{array}$ \\
\hline WT 6c-4/+ 1147 & 573.5 & 564 & 96.7 & \\
\hline
\end{tabular}

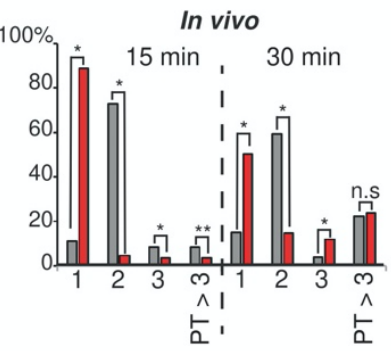

larp $6 c-3, n=189$

WT, $n=217$
E

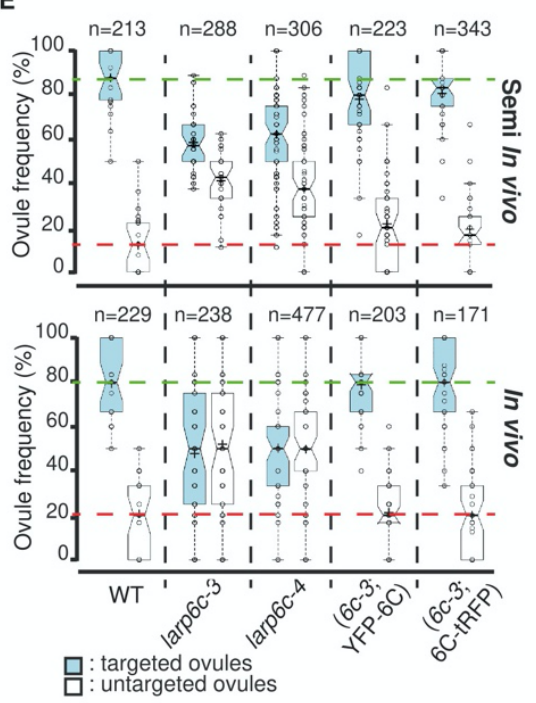

F

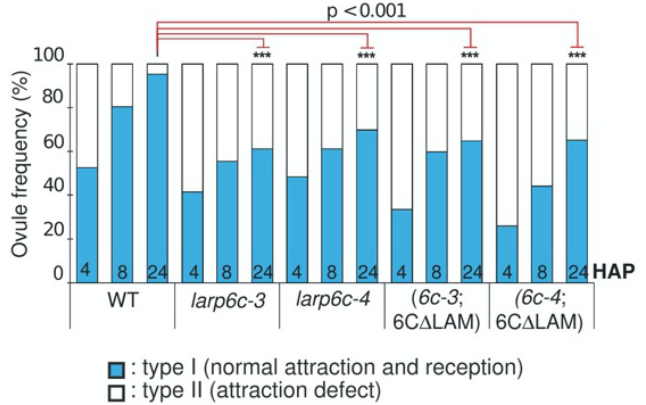

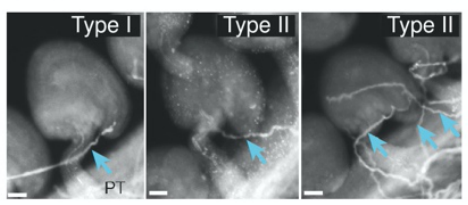

Figure 1: LARP6C is required for pollen tube guidance. (A), Immunoblot analysis of steady-state LARP6C protein accumulation. The same blot was probed with antibodies against LARP6C or ACTIN as a loading control. (B), larp6c-3 and larp6c-4 alleles are lossof-function mutants. Top panel: schematic representation of the LARP6C gene and LARP6C protein. Plain boxes represent exons and lines represent introns. The colour code for exons is identical to that for the conserved protein domains. The insertion sites of the T-DNAs in larp6c-3 and 6c-4 mutants are reported. Bottom panel: immunoblot analysis of LARP6C accumulation in flowers from wildtype, larp6c-3 and 6c-4 lines. ACTIN was used as a loading control. (C), Transmission efficiencies of larp6c-3 and $6 c-4$ mutant alleles. Transmission Efficiency (TE) was calculated as: (([No of mutant] / [No of wild type]) x 100). ns: the number of mutant seedlings in the progeny is not significantly different from the expected number of mutant seedlings. (D) Scoring of germination rate, pollen tube bursting, and elongation in vitro and in vivo. Left panels show representative images of in vivo (upper part) and in vitro (lower part) experiments. On the right, graphs report the frequency of ungerminated pollen (1), PT emergence (2), PT burst (3), and PT longer than three lengths of the pollen grain $(P T>3) 15$ or 30 minutes after pollination. $n$ represents the number of scored pollen grains. (E), Semi in vivo (SIV) (upper panel) and in vivo (bottom panel) pollen tube guidance assays. For SIV assays, wild-type ovules were arranged around homozygous ms1 pistil explants pollinated with pollen from various homozygous backgrounds. For in vivo assays, homozygous $m s 1$ pistils were pollinated in planta with pollen from various homozygous genotypes. For each type of experiment, the numbers of targeted (blue boxes) and non-targeted (white boxes) ovules were scored and results represented as whisker notched boxplots. The genotypes of the pollen used for fertilization are recorded below the graphs and $n$ is the total number of ovules scored in each experiment. The dotted lines respectively show the wild type mean values of targeted (blue) and non-targeted (dark red) ovules by wild-type pollen. (F) In vivo scoring of pollen tube behaviours. Wild-type pistils were pollinated in planta with pollen from various homozygous genotypes (recorded below the graph) and the behaviour of pollen tubes monitored and scored over a time course: $4 \mathrm{~h}, 8 \mathrm{~h}$ and $24 \mathrm{~h}$ after pollination (HAP). Type-I (blue bars): targeting and reception are normal, Type-II (white bars): attraction (targeting) is defective. Below the graph are representative images of the type of behaviours observed. Scale bars correspond to 10 $\mu \mathrm{m}$. The table below the graphs report the numbers of scored ovules $(\mathrm{n})$. p-values were calculated with an unpaired Student $\mathrm{t}$-test. ns: not significant, ${ }^{*}$ : $p$-value $\leq 0.05,{ }^{* *}: p$-value $\leq 0.01,{ }^{* *}: p$-value $\leq 0.001$. 
A

B

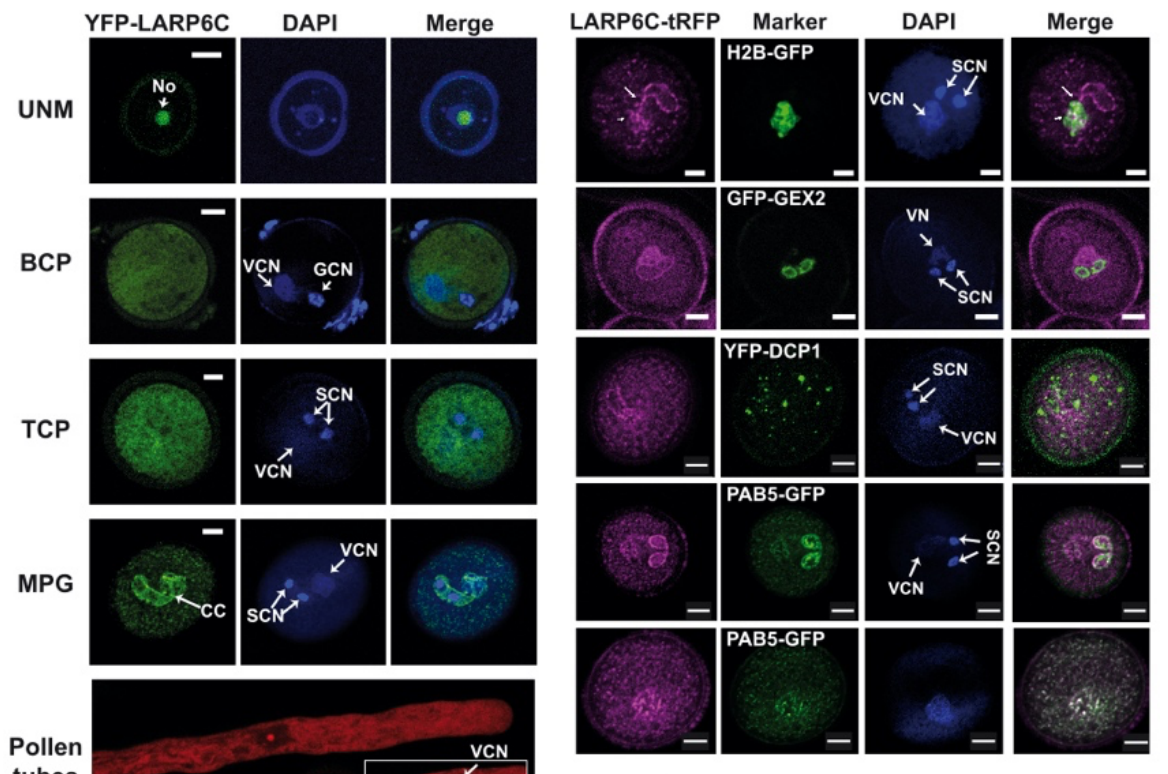

tubes

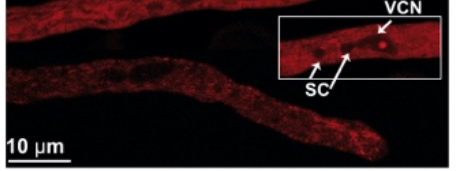

LARP6C-tRFP

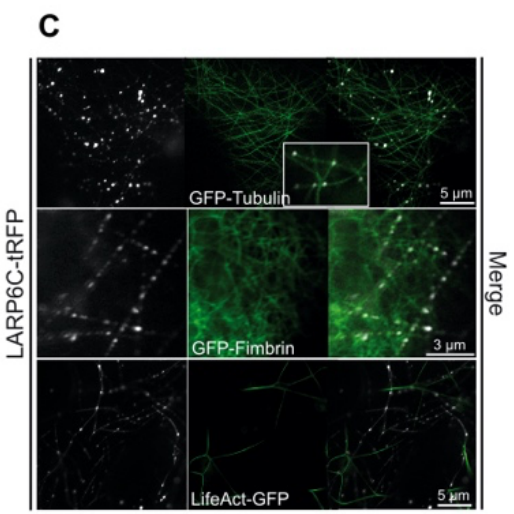

Figure 2: Subcellular distribution of LARP6C across pollen development and pollen tubes. (A) Subcellular localization of fluorescently tagged YFP-LARP6C by confocal microscopy at different stages of pollen maturation: UNM: uninucleate microspore, BCP: bicellular pollen, TCP: tricellular pollen, MPG: mature pollen grain and Pollen tube. Scale bars correspond to $5 \mu \mathrm{m}$ for pollen and $10 \mu \mathrm{m}$ for pollen tube images. Representative images of LARP6C fused at its C-terminus with a tagRFP reporter are shown in Supplemental Figure S5A. (B) Confocal observation of mature pollen grains that stably co-express the LARP6C-tRFP fusion and the H2B-GFP fusion, the GFP-GEX2 fusion, the YFP-DCP1 fusion or the PAB5-GFP fusion. Except for the H2B-GFP marker, which is expressed from the tomato LAT52 promoter, all marker genes are expressed from their own upstream genomic sequences. Scale bars correspond to $5 \mu \mathrm{m}$. In (A) and (B), arrows indicate the position of the Nucleolus (No), Generative Cell Nucleus (GCN), Vegetative Nucleus (VCN), Sperm Cell Nucleus (SCN) and Cytoplasmic Connection (CC). (C) Confocal observation of N. benthamiana epidermis cells transiently expressing the LARP6C-tRFP fusion co-expressed with microtubule-binding GFP-AtTUB6 and two actin-binding GFP-FABD2 or LifeAct-GFP reporters. $n=20$ cells from three independent infiltration repeats. 

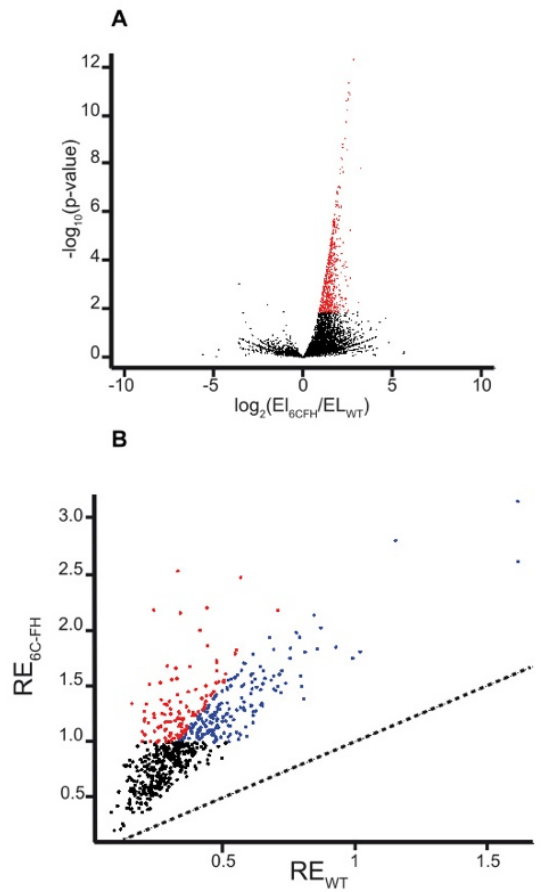

C

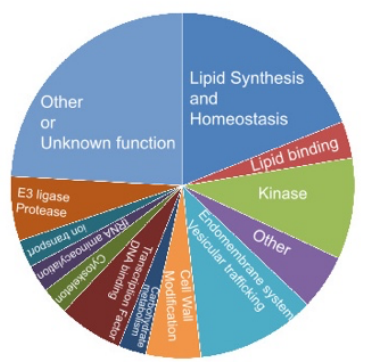

Figure 3: RIP-Seq identification of LARP6C mRNA targets. (A) Volcano plot repartition of the log2 of fold changes between values in the eluate fractions of LARP6C-FH and wildtype for mRNAs found in the LARP6C-FH eluate fraction, according to their p-values obtained through the DESeq2 pipeline. Red dots represent mRNAs that are significantly more represented in LARP6C-FH eluates. A p-value $\leq 0.01$ and $F C \geq 1$ was used as significance criteria. (B) Plotting the RE (Eluate-RPM/Input-RPM) values in LARP6C-FH against the RE values in wild type for the 635 mRNAs highlighted in graph A. mRNAs with a RE6c-FH $\geq 3 R E-W T$ are labelled as blue dots. See Supplemental Figure S6C for a detailed description of the RIP-seq pipeline. (C) Pie chart representation of the number of LARP6C targets within the various functional categories identified. 


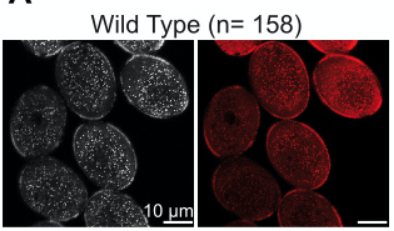

Bodipy

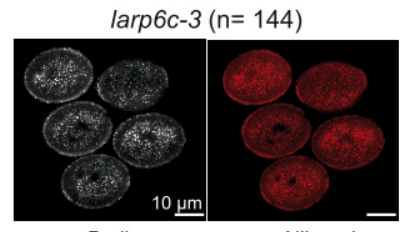

Bodipy

Nile red

\section{C semi in vivo PTs}

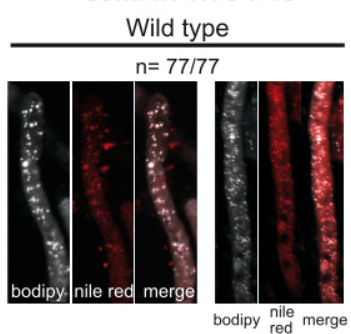

larp6c-3

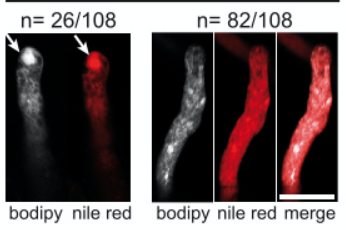

E

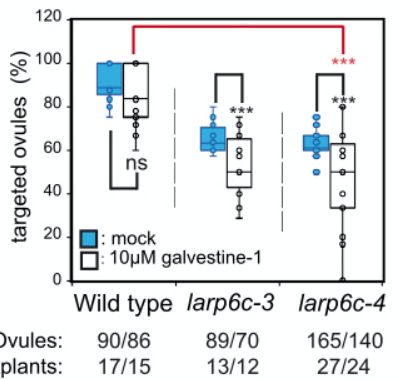

Figure 4: Loss of LARP6C function affects lipid homeostasis in pollen tubes. Wild-type and larp6c-3 pollen grains were stained with Bodipy 505/515 and Nile red at the Mature Pollen Stage (MPG) (A) or in pollen tubes (PTs) (B, C). In (B) PTs were germinated and grown in vitro. PTs were collected at $2 \mathrm{~h}$ and $4 \mathrm{~h}$ after activation for staining. Representative images are shown. In (C) PTs grown semi in vivo were collected $7 \mathrm{~h}$ following pollination (4 hap, pistils are cut at shoulder, placed on plate with ovules and further incubated 3 hours before scoring). $\mathrm{n}$ represents the number of pollen tubes with LDs distributed as on the presented image over the total number of observed pollen grains. Scale bars correspond to $10 \mu \mathrm{m}$ in (A) and to $5 \mu \mathrm{m}$ in (B, C). (D), Monitoring of pollen tube growth in the presence of galvestine-1. Whisker boxplot representation of the length of wild-type or larp6c-3 pollen tubes grown in vitro for 4 hours in the presence or absence of galvestine-1. 1: untreated, 2: mock (1\% DMSO), 3 and 4: $5 \mu \mathrm{M}$ and $10 \mu \mathrm{M}$ galvestine-1. (E) Semi in vivo pollen tube guidance assays. Assays were conducted either in the presence of $1 \%$ DMSO (untreated) or in the presence of 10 $\mu \mathrm{M}$ of galvestine-1. Results are represented as whisker boxplots. p-values were obtained through an unpaired Student t-test, ns: not significant, ${ }^{* * *}$ : $p$-value $\leq 0.001$. 
A
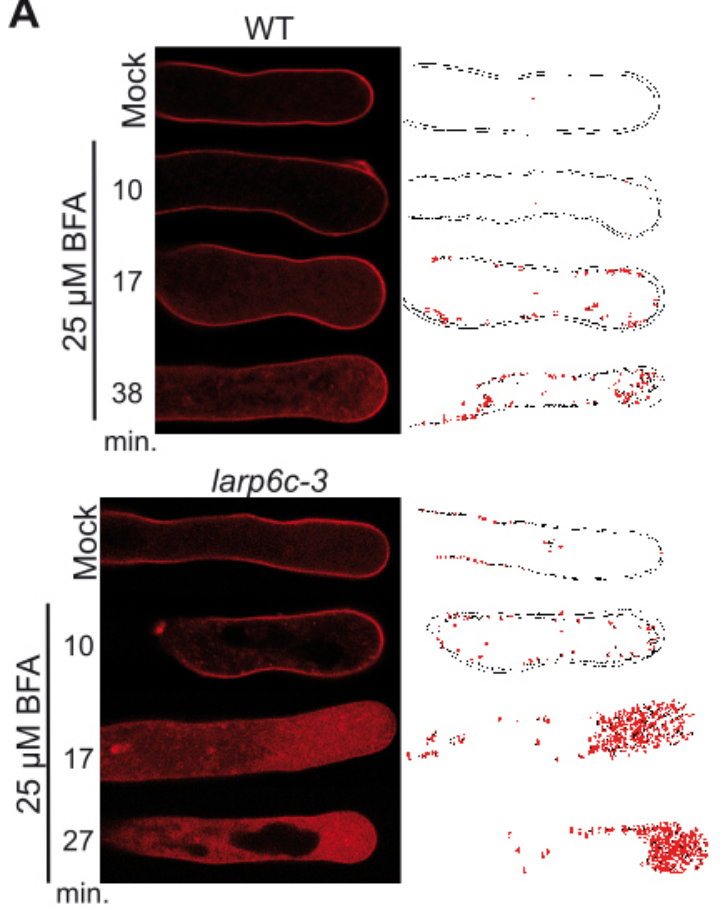

B

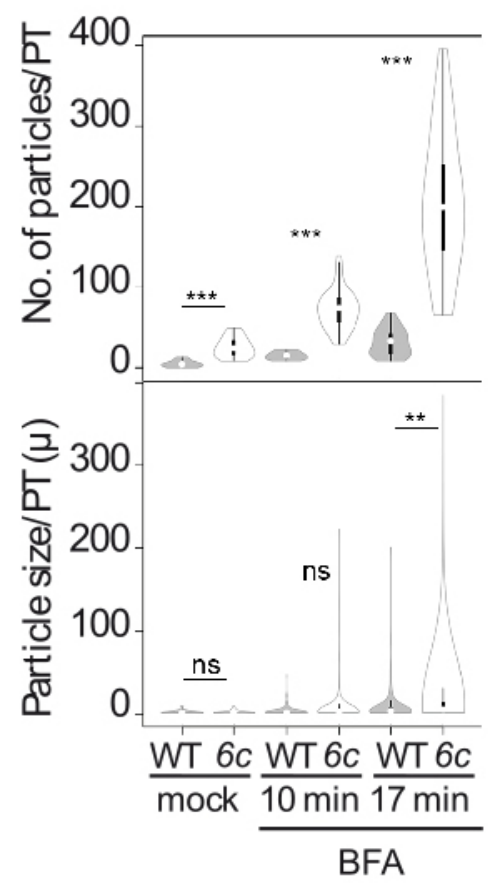

Figure 5: Pollen tubes deprived of LARP6C are hypersensitive to Brefeldin A (BFA). Pollen grains were germinated in vitro and after $1 \mathrm{~h}$ activation on germination medium, pollen tubes were incubated with mock (DMSO) or $25 \mu \mathrm{M}$ BFA. (A) Representative images of FM4-64 staining captured at the respective times or wild type (WT) and larp6c-3 PTs treated with mock or BFA. Sketched outline of the PTs with red dots (counts) describing the appearance of the particle frequency and size, as detected by the ImageJ particle analysis plug in. (B) Following FM 4-64 staining, the number (top panel) and size (bottom panel) of BFA bodies was scored over a time course and represented as violin plots. Number of PTs observed to score particle number: WT: mock: 23, 10 min: 14,17 min: 30; larp6c-3: mock: 32, $10 \mathrm{~min}: 35,17 \mathrm{~min}: 25$. Number of PTs observed to score particle size: WT: mock: $59,10 \mathrm{~min}: 62,17 \mathrm{~min}:$ 95; larp6c-3: mock: 61, 10 min: 115, 17 min: 189. p-values were obtained with an unpaired Student t-test, ns: not significant, **: pvalue $\leq 0.01,{ }^{* * *}: p$-value $\leq 0.001$ 


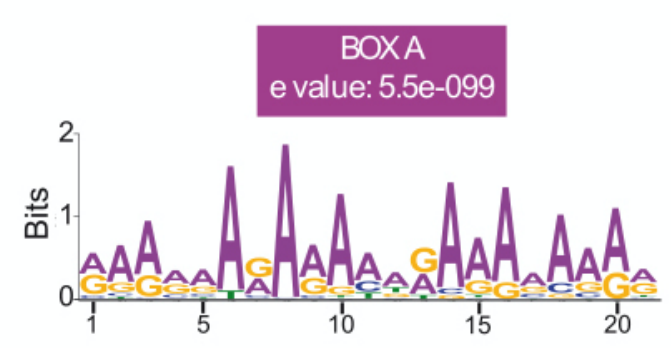

B

B1: 5'-CUUCUUUCUUUCUUUUUUGUU-3'

B2: 5'-UCUCUCUGUUUCUUAUUCAUC-3'
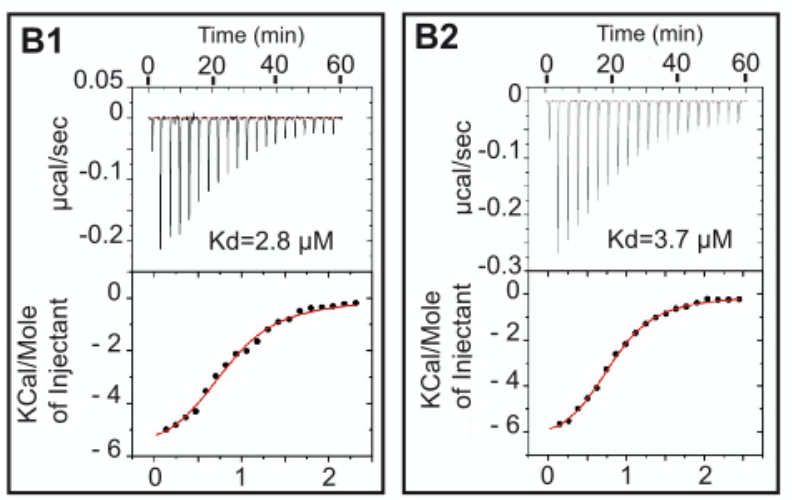

D

B3: 5'-CCUCUCCCCCCCUCCCCCCUC-3'

B4: 5'-CCUCUCUCCUUCUCCUCCCUC-3'
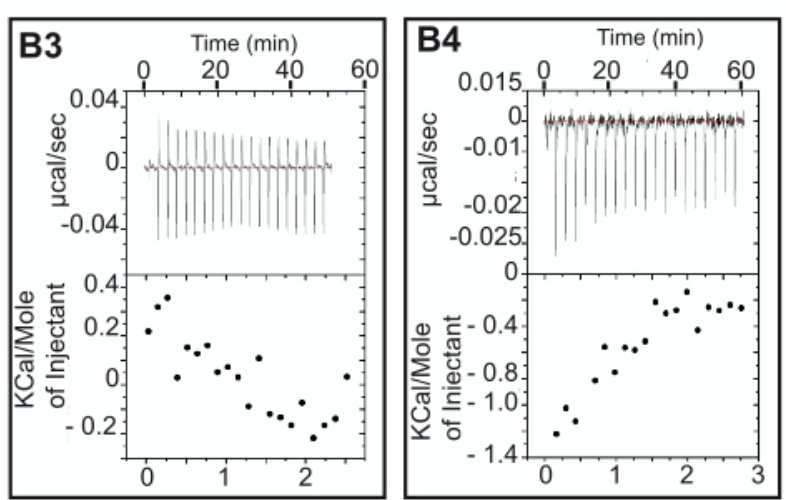

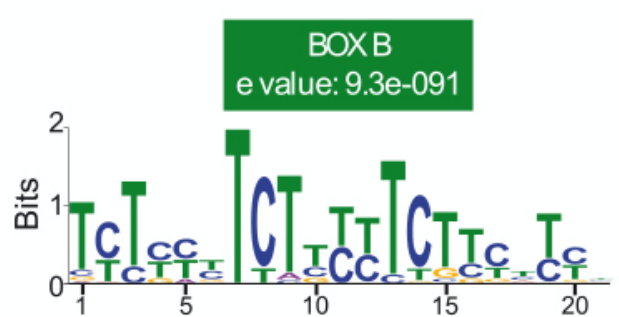

- tRNA
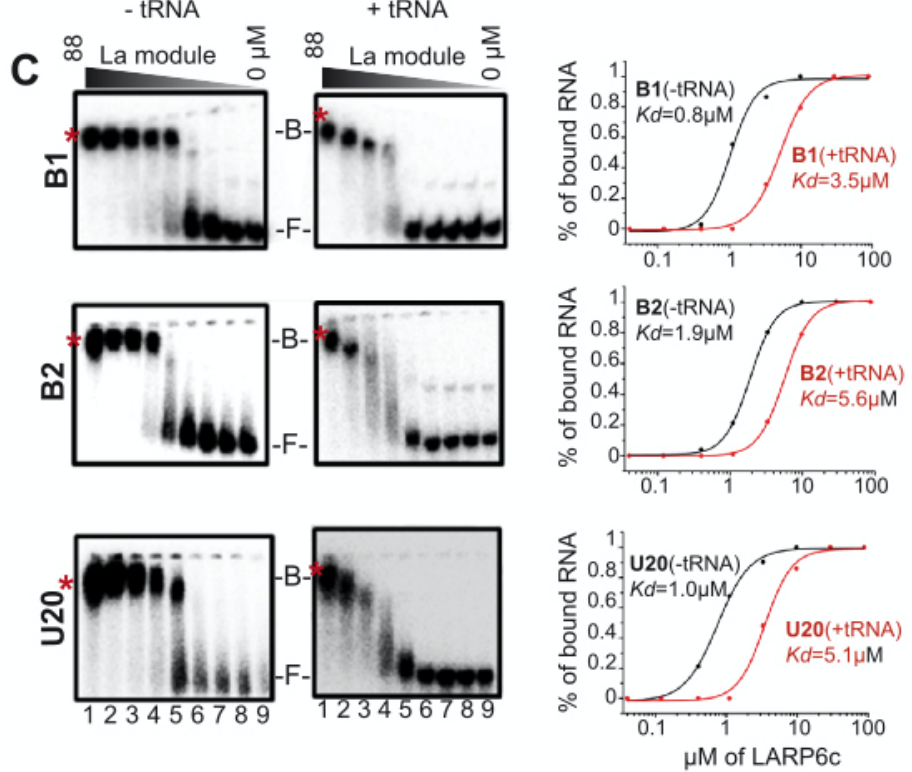

E

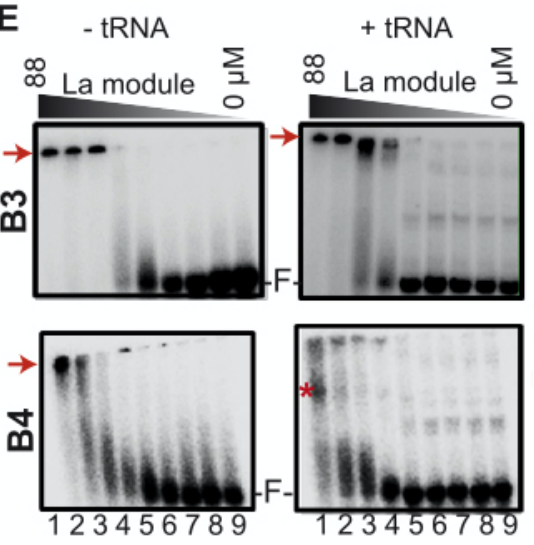

Figure 6: The LARP6C La-module binds to B-type RNA boxes. (A), Consensus sequences of the conserved motifs within the 5'UTRs of LARP6C-bound mRNAs. Calorimetric (B, D) and EMSA (C, E) analyses of the interaction between the LARP6C La-module (encompassing residues 137-332) and oligos B1, B2 (B, C), $\cup_{20}(C)$, B3 and B4 (D, E). In (B) and (D): for each graph, the upper panel corresponds to the raw titration data showing the thermal effect of injecting an RNA oligo solution into a calorimetric cell containing the recombinant LARP6C La-module. The lower panels show the normalized heat per injection values obtained by integrating the raw data and subtracting the heat value of the RNA dilution. The red lines in the graphs for oligos B1 and B2 (B) represent the best fit derived by a non-linear best-square procedure based on an independent binding site model. The dissociation constants (Kd) are indicated for the B1 and B2 oligos; the thermodynamic parameters are shown in Supplemental Table S1. (C, E), EMSAs of LARP6C La-module binding to: $B 1, B 2$ or oligo $U_{20}(C)$, B3 or B4 (E). Decreasing concentrations $(\mu M)(88$ (lane 1), 29.3 (lane 2), 9.8 (lane 3), 3.3 (lane 4), 1.1 (lane 5), 0.4 (lane 6), 0.12 (lane 7), 0.04 (lane 8) and 0 (lane 9)) of the recombinant LARP6C La-module were mixed with $3 \mathrm{nM}$ of 5'-labelled oligos. B stands for Bound, $\mathrm{F}$ for Free, red asterisks mark the RNA-protein complex, and the red arrows on panels $E$ shows samples retained into the gel wells. Experiments were conducted in the absence (-tRNA) or presence (+tRNA) of unlabelled competitor (tRNAmix of E. coli MRE 600 at $0.01 \mathrm{mg} / \mathrm{mL}$ concentration). Graphs in panel C show the quantification of the bound RNA fraction versus the protein concentration in the absence (black lines) or presence (red lines) of tRNA competitor. The values of the dissociation constants are reported. Kd values reported for the EMSA experiments were calculated out of three independent replicates with the following standard deviations: LARP6C-B1 (-tRNA): 0.8 +/- 0.1; LARP6C-B1 (+tRNA): 3.5 +/- 0.3 ; LARP6C-B2 (-tRNA): 1.9 +/- 0.3; LARP6C-B2 (+tRNA): 5.6 +/- 0.2. 
A

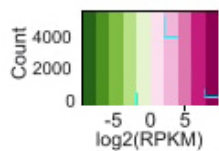
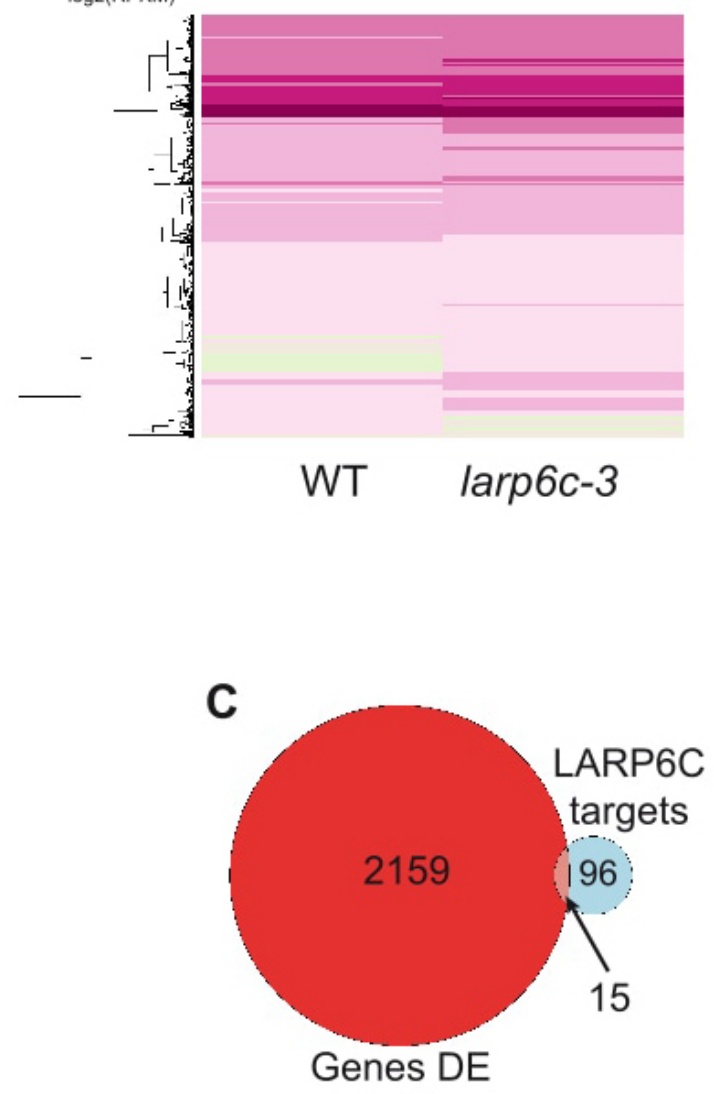

B
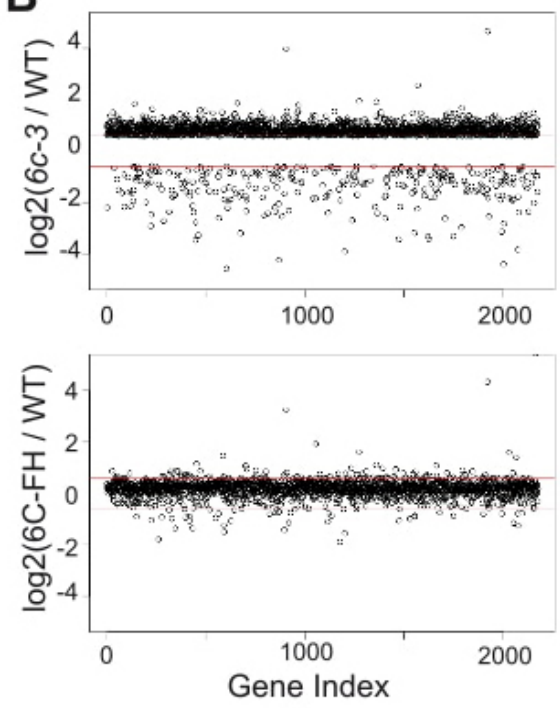

D

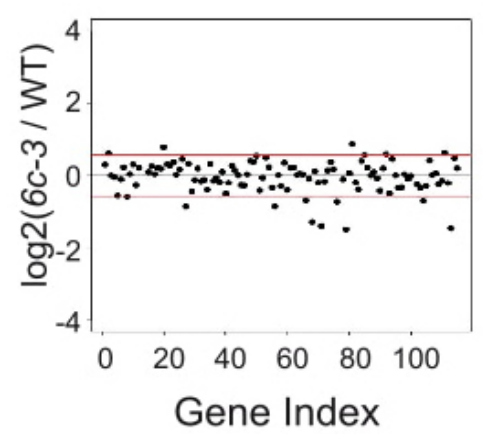

Figure 7: larp6c loss-of-function does not affect the steady-state transcript levels of its target in dry pollen. (A), Heat map representation of the $\log _{2}($ RPKM) values of genes that are differentially expressed between wild type (WT) and larp6c-3 plants. The heat map was built from the list of DE genes with log2(RPKM) values between -2 and +10 (2142 genes: $98.5 \%$ of the DE genes). (B), Plot representation of the log2 values of the ratios: (larp6c-3/wild type) (upper panel) and log2(6C-FH/wild type) (lower panel) for the 2174 genes found to be differentially expressed in larp6c-3 mutant pollen. Red lines mark the cut off value $(\log 2(1.5)$ and $\log 2(1 / 1.5))$. (C) Venn diagram representation of the number of transcripts that are DE in larp6c-3 and/or immunoprecipitated by 6C-FH. Note that of the 115 RIP targets, 19 were not present in the transcriptomic data from RNA-seq. (D) plot representation of the log2(larp6c-3/WT) for mRNAs identified by RIP-seq. Red lines mark the cut off value $(\log 2(1.5)$ and $\log 2(1 / 1.5))$. 

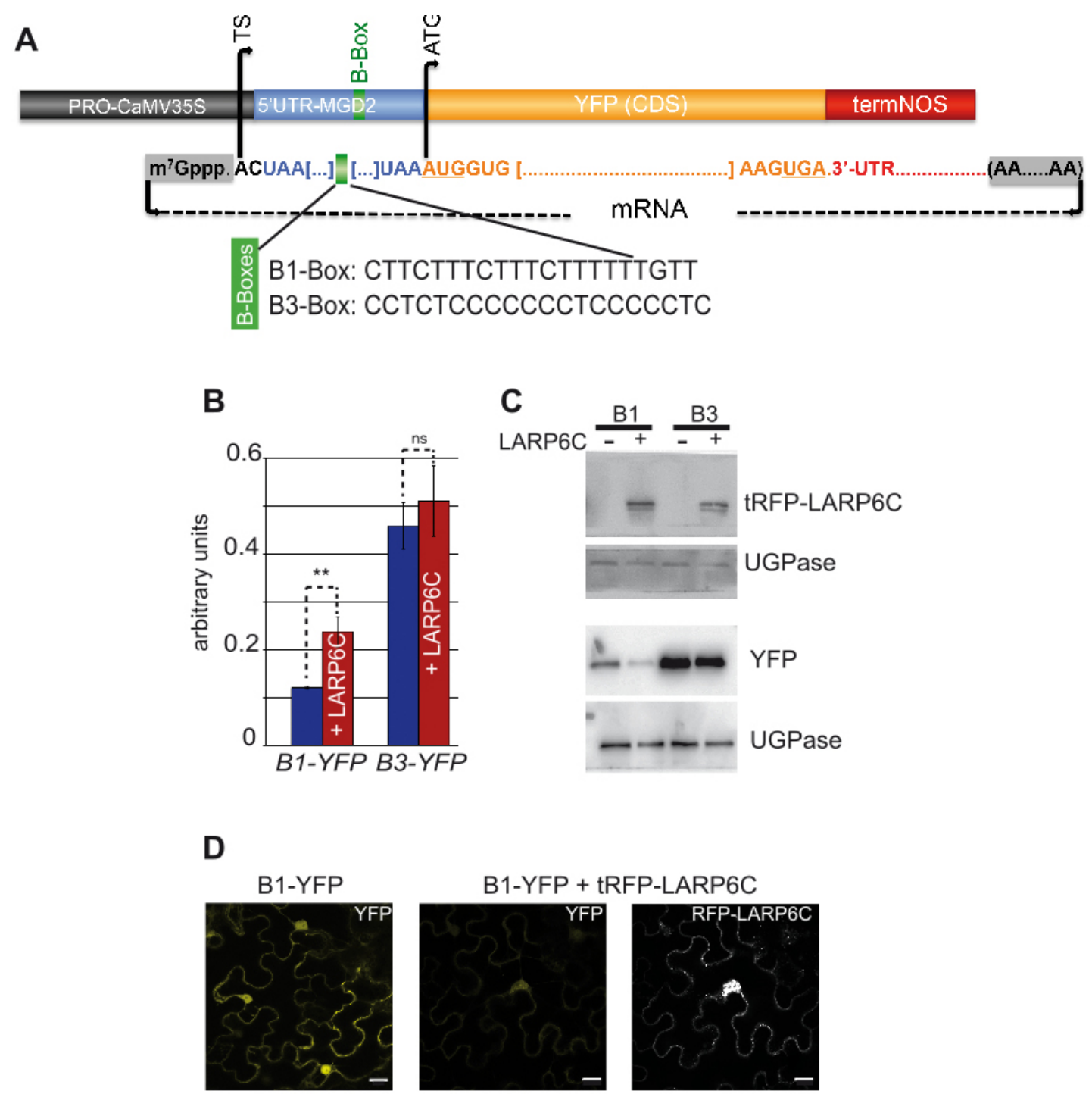

\section{B3-YFP}

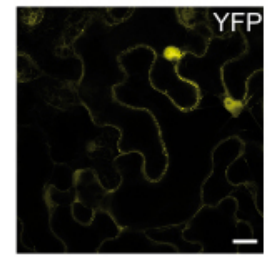

B3-YFP + tRFP-LARP6C

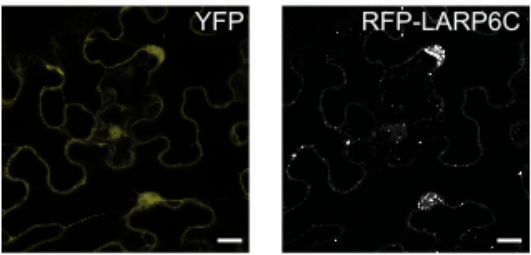

Figure 8: LARP6C binding at the 5'UTR of a reporter construct reduces protein and increases mRNA levels. (A) Schematic representation of the YFP reporter constructs, (B) RT-qPCR monitoring of YFP mRNA levels. To normalize YFP mRNA levels to transformation efficiency, we used the levels of HPTII mRNA encoded by the HPTII gene carried by the YFP binary plasmid but not the tRFP-LARP6C one. SDs were calculated from three biological replicates. $p$-value were obtained using a Student-t test. ${ }^{* *} P<$ 0.005 , ns: not significant. (C) Immunoblot analysis of tRFP-LARP6C and YFP protein levels. Two immunoblots were prepared and respectively hybridized with anti-LARP6C or GFP antibodies. Levels of UGPase were used as a loading control. Representative images of three replicates are shown. (D) Confocal imaging of YFP from leaves not transformed with tRFP-LARP6C (left panels) and of YFP and tRFP-LARP6C distribution (right panels). Scale bars represent $20 \mu \mathrm{m}$. Leaves that were observed are different from those used to prepare total RNA and protein extracts. Representative images of three biological replicates are shown. 

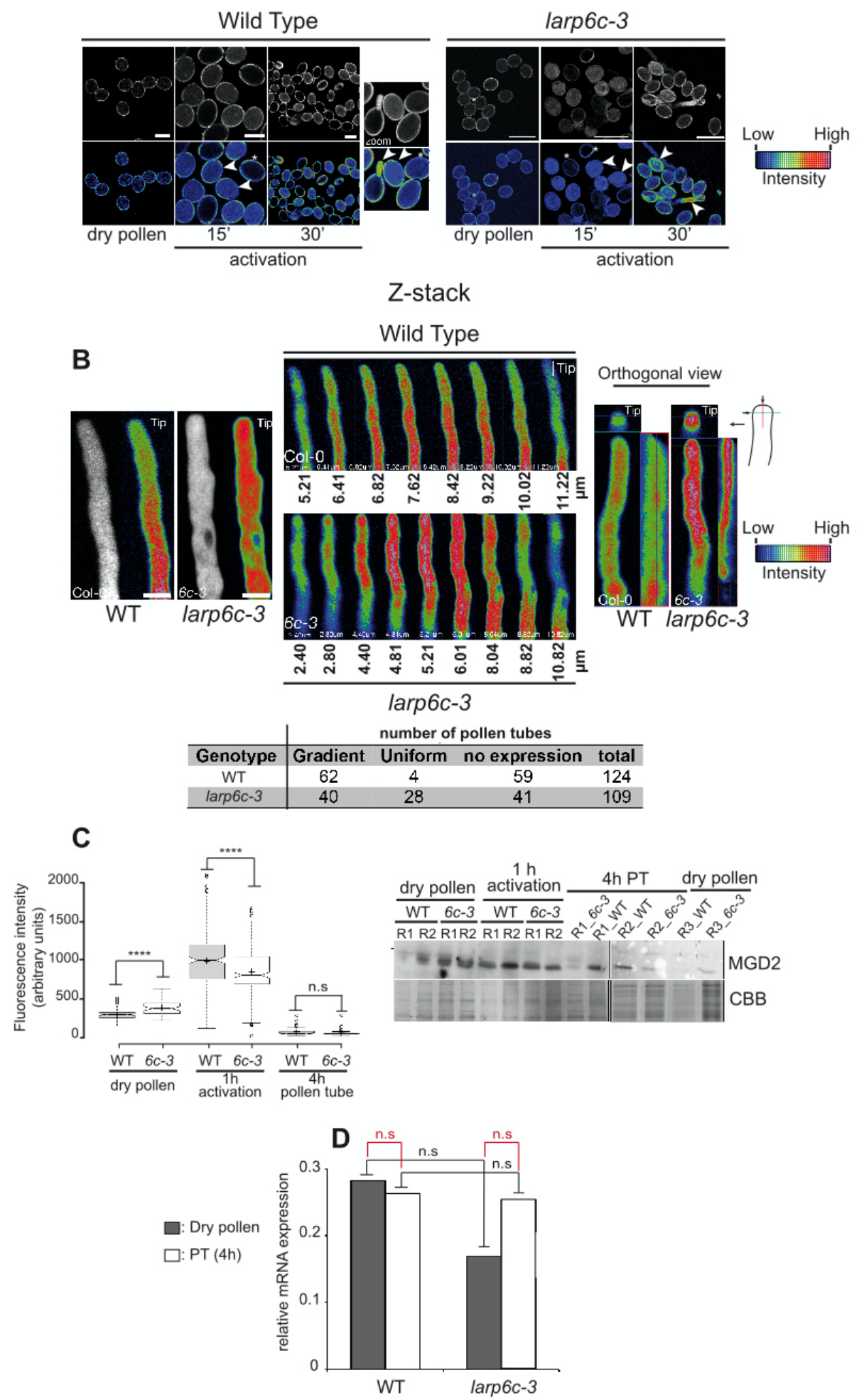

Figure 9: LARP6C is involved in the dynamic fine-tuning of MGD2 protein accumulation in vivo. Monitoring of the levels of MGD2-YFP fusion protein expressed from the MGD2 native promoter (PROMGD2:MGD2-YFP) and endogenous MGD2 in wild type (WT) and larp6c-3 plants. (A) Representative images of YFP signal in heterozygous mature pollen grains at activation: 0,15 and 30 min after incubation on in vitro germination medium. Bottom panels show images in pseudo color with the associated scale bar representing the intensity of the YFP signal. White arrows and asterisks respectively point to segregation pollen grains expressing or not expressing the translational fusion. (B) Live cell imaging of MGD2-YFP distribution in pollen tubes. The numbers of pollen tubes with gradient MGD2 distribution toward the tip, uniform (lost gradient), or no expression of MGD2-YFP are reported in the table below. Scale bars correspond to $10 \mu \mathrm{m}$. (C) Left panel: fluorescence quantification of MGD2-YFP in wild type (WT) and larp6c-3 (6c-3) pollen and during the progamic phase $(1$ and $4 h) . n=3$ biological replicates at each time point, a Student t-test for two independent samples/two-tailed test was conducted, with ns: not significant, ${ }^{* * * *} p$-value $\leq 0.0001$. Right panel: immunoblot analysis of endogenous levels of MGD2 in pollen and during progamic phase in wild type and larp6c-3. Experiments were conducted in two or three independent replicates labelled R1, R2, and R3. (D) RT-qPCR analysis of MGD2 mRNA levels originating from the MGD2-YFP transgene and endogenous MGD2 gene in dry pollen and $4 \mathrm{~h}$ pollen tubes. Experiments were conducted in three independent replicates, and TUBULIN8 mRNA was used as a control. An unpaired Student t-test was conducted, with n.s.: not significant. Both wild type and larp6c-3 express MGD2-YFP from the same primary transformant (line 6). In A and B, the MGD2-YFP transgene is in the heterozygous state and the larp6c-3 allele is homozygous. In C and D, wild type and larp6c-3 carry the MGD2-YFP transgene in the homozygous state. 
Supplemental data. Billey, Hafidh et al. (2021). LARP6C orchestrates post-transcriptional reprogramming of gene expression during hydration to promote pollen tube guidance. Plant Cell.

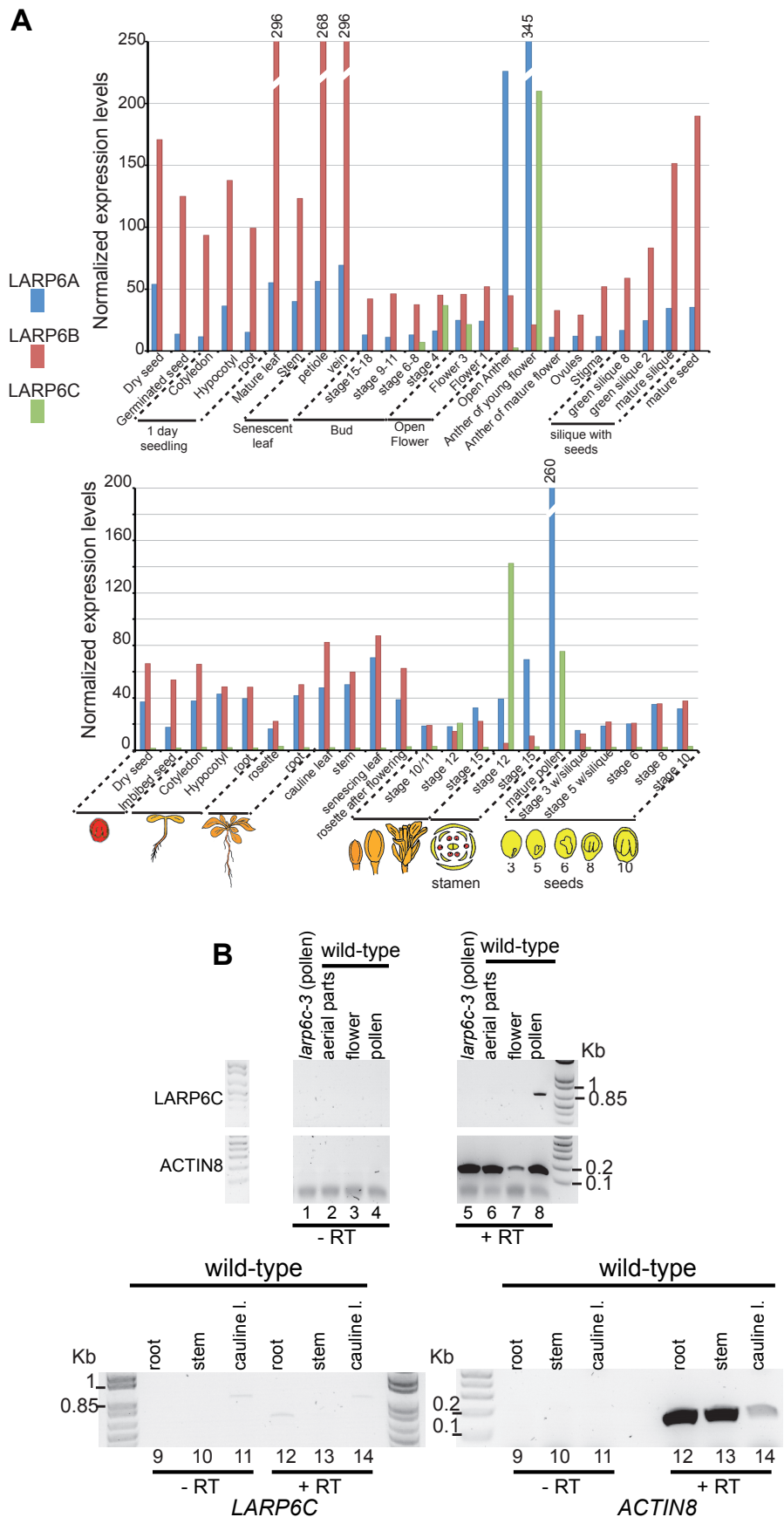

Supplemental Figure S1: Expression profiles of LARP6A, 6B and 6C mRNAs across development. (Supports Figure 1A). (A), Expression profiles of the LARP6A (AT5g46250), 6B (AT2g43970) and 6C (AT3g19090) mRNA across Arabidopsis development. Data were retrieved from the TraVa database of gene expression profiles monitored by RNA-seq (http://travadb.org/) (Klepikova et al., 2015) (upper graph) and from the Arabidopsis eFP Browser from the Bio-Analytic Resources for plant biology (http://bar.utoronto.ca/) (Winter et al., 2007) and are represented as histograms. The normalized expression values are arbitrary units. (B) RT-PCR analysis of steady-state LARP6C mRNA levels in various tissues from wild-type plants. Specificity of the PCR reactions was verified by omitting the reverse transcription step (no RT, lanes 1-4; 9-11). RNAs from larp6c3 pollen grains were utilized as negative controls (lanes 1 and 5). RNAs extracted from: seedling aerial parts (lanes 2, 6), whole flower (lanes 3,7), mature pollen grains (lanes 4, 8), seedling roots (lanes 9, 12), stem (lanes 10, 13) and cauline leaves (lanes $11,14)$ were tested. The ACTIN8 transcript was used as a positive control. 
Supplemental data. Billey, Hafidh et al. (2021). LARP6C orchestrates post-transcriptional reprogramming of gene expression during hydration to promote pollen tube guidance. Plant Cell.

A
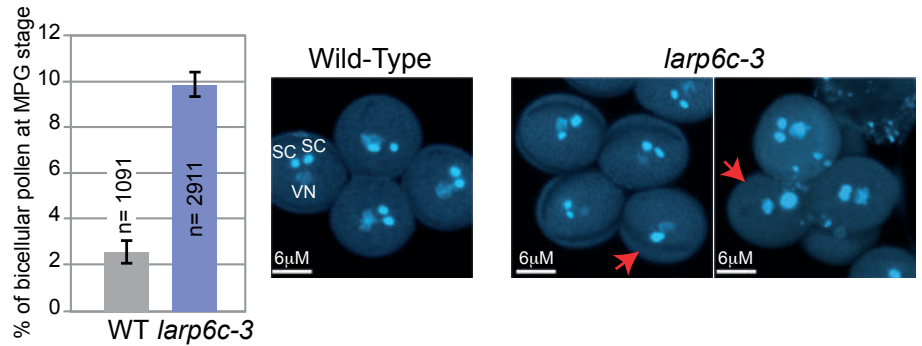

B

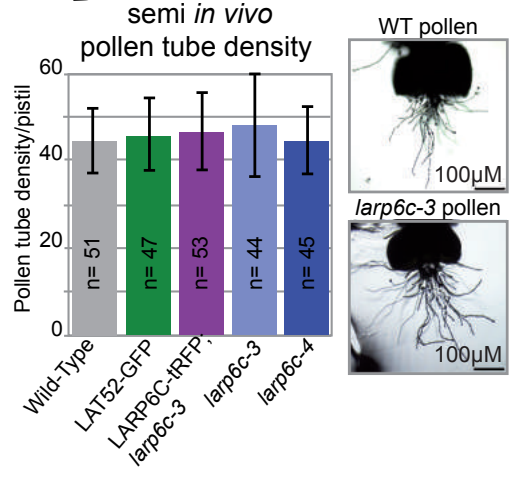

C

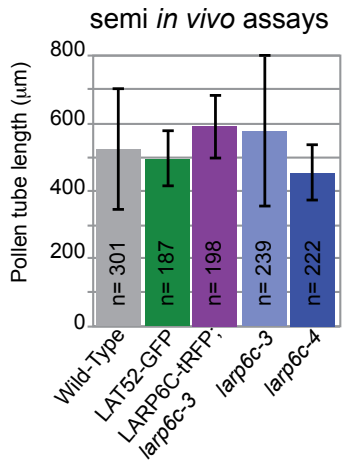

D

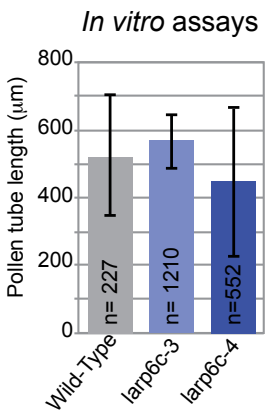

E

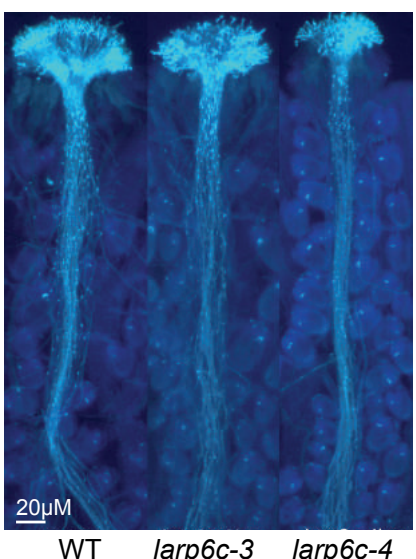

Supplemental Figure S2: Pollen maturation, germination, and pollen tube growth of larp6c loss-of-function mutants. (Supports Figure 1). (A), larp6c loss-of-function induces a slight pollen maturation deficiency. Mature Pollen Grain (MPG) from wild type (WT) or larp6c-3 homozygous in quartet (qrt-/-) mutant plants were collected and observed following DAPI staining. The number of bicellular pollen grains was scored over the total number of observed MPGs. The results are presented as histograms and correspond to mean values of three replicates $\pm S D$ and " $n$ " is a total number of pollen grains examined. On the right are representative fluorescence micrographs of wild-type and larp6c-3 pollen grains stained with DAPI. Red arrows point to mature pollen grains in which the generative cell remained undivided and appeared as binucleate. Scale bars correspond to $6 \mu \mathrm{m}$. (B), Mean germination rates $( \pm$ SD) of pollen tubes emerged per pistil in a semi in vivo assay. The total number of observed pistils is reported as " $n$ " from three biological replicates. Transgenic LAT52-GFP pollen grains were used as a control. (C), Pollen tube length derived from the semi in vivo assay. No significant difference was observed between wild-type and mutant pollen tubes (Student $t$ test $\mathrm{p}>0.05$ ) calculated from pooled replicates. (D), In vitro pollen tube length measurements. The total number of pollen tubes measured is reported on the histogram as "n". (E) In vivo aniline blue staining of pollen tubes after fertilization with wild-type (WT), larp6c-3 or larp6c-4 pollen. $n=10,16$ and 21 pistils respectively. In the semi in vivo assays, scoring was conducted 7 hours after pollination (hap) (4 hap pistils were cut at the shoulder, placed on a plate, and further incubated 3 hours before scoring). In the in vitro assays, scoring was conducted 4 hours after activation on germination medium. In the in vivo assays, scoring was performed 24 hap. 
Supplemental data. Billey, Hafidh et al. (2021). LARP6C orchestrates post-transcriptional reprogramming of gene expression during hydration to promote pollen tube guidance. Plant Cell.
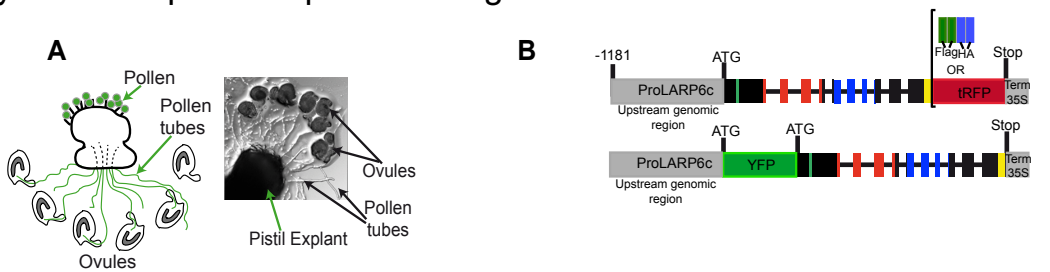

C

LARP6C-RFP // YFP-LARP6C

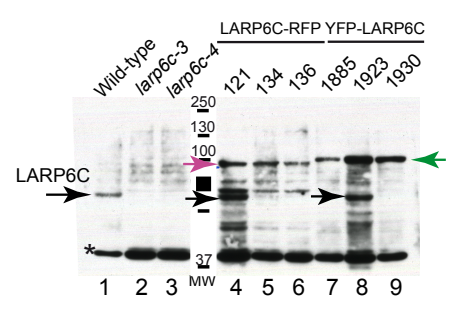

LARP6C-FlagHA
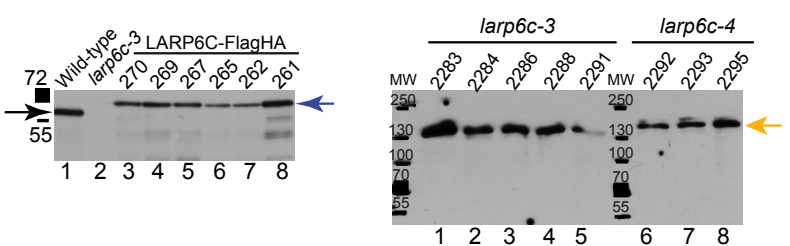

D
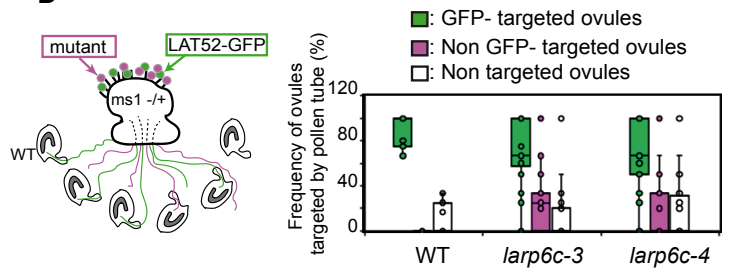

Nb of Ovules: $\quad 153 \quad 62 \quad 149$

Nb of Pistil Explants: $49 \quad 24 \quad 46$

$\mathbf{F}$

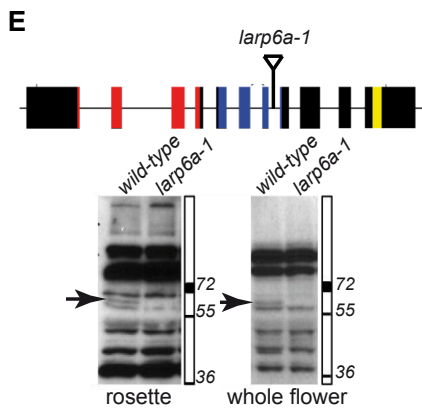

\begin{tabular}{ccccccc}
\hline Parental Genotype & $\begin{array}{c}\text { Progeny } \\
\text { (total no) }\end{array}$ & $\begin{array}{c}\text { No of } \\
\text { expected } \\
\text { mutants }\end{array}$ & $\begin{array}{c}\text { No of } \\
\text { observed } \\
\text { mutants }\end{array}$ & $\begin{array}{c}\text { Transmission } \\
\text { efficiency (\%) }\end{array}$ & $\begin{array}{c}\text { khi2;p- } \\
\text { value }\end{array}$ \\
\hline Male & Female & & & & & \\
$6 \mathrm{a}-1 /+$ & WT & 973 & 486.5 & 475 & 95.4 & $0.54 ; \mathrm{ns}$ \\
$\begin{array}{c}6 c-3 /+ \\
6 \mathrm{a}-1 / 6 \mathrm{a}-1\end{array}$ & WT & 634 & 317 & 287 & 82.7 & $5.68 ;<0.05$ \\
\hline
\end{tabular}

G

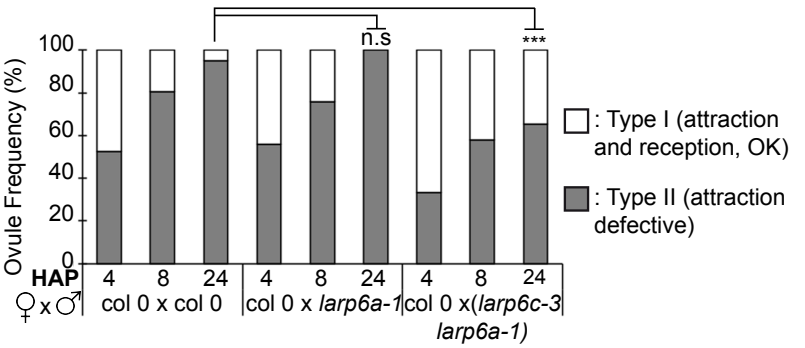

Supplemental Figure S3: Schematic representation of LARP6C transgenes and immunoblotting analyses of their accumulation. Semi in vivo pollen tube guidance competition assays. Analysis of LARP6A function in male fertilization. (Supports Figures 1 to 3). (A) Representation of the setting of a semi in vivo guidance assay. (B) Schematic representation of the transgenes utilized to stably express tagged versions of LARP6C protein in mature pollen. The drawing and color codes are as in Figure 1B. (C) Immunoblot analysis of the expression of tagged LARP6C transgenic proteins. Total proteins were extracted from whole flowers, and blots were probed with anti-LARP6C antibodies. Left panel: expression levels of LARP6C-tRFP and YFPLARP6C in the wild type (lanes 4 and 8), larp6c-3 (lanes 5, 6, 9) and/or larp6c-4 (lane 7) backgrounds. Confocal analyses presented in Figure 2 were conducted with pollen from: lines 1885 (UNM), 1930 (BCP and TCP), 1923 (MPG) and 136 (pollen tubes). Line 134 was used to co-express the various markers presented in Figure 3. Lines 134 and 1930 were used as complemented lines for pollen experiments reported in Figures 1D and E and Supplemental Figure 2. Black arrows point to endogenous LARP6C, red arrows to LARP6C-tRFP, green arrows to YFP-LARP6C and black asterisk to non-specific signal. Middle panel: steady-state levels of the LARP6C-FlagHA (6C-FH) fusion from six distinct transformants (lanes 3 to 8 ). $6 \mathrm{C}$-FH was expressed in larp6c-3 knockout plants. Mature pollen from line number 267 (lane 5) was used for the RIP-seq experiment. Right Panel: expression levels of YFP-LARP6C-ALAM expressed in the larp6c-3 (lanes 1-5) or larp6c-4 (lanes 6-8) backgrounds. Complementation assays presented in Figure 1F were conducted with lines 2291 (lane 5) and 2293 (lane 7). (D) Semi in vivo pollen tube guidance competition assays. On the left, a cartoon representation of the pollen tube guidance assay. Unfertilized wild-type ovules were arranged around an $m s 1$ pistil pollinated with both mutant larp6c and LAT52-GFP pollen. A limited pollination was performed with pollen from wild-type plants homozygous for LAT52-GFP reporter (hmLAT52-GFP) (labeled "WT"), with hmLAT52-GFP and larp6c-3 pollen (labeled "larp6c-3") or with hmLAT52-GFP and larp6c-4 pollen (labeled "larp6c-4"). Ovules were scored as: GFP positive (targeted by LAT52-GFP pollen), non-GFP ovules (targeted by mutant pollen) or non-targeted. The results are presented as whisker boxplots. (E) Top panel: Schematic representation of the LARP6A genomic locus; the color code is identical to that of Figure 1B. The position of larp6a-1 (SK39668) T-DNA is reported. Bottom panel: immunoblot analysis of LARP6A steady-state levels in the wild-type and larp6a-1 backgrounds. (F) Male transmission efficiencies of larp6a-1 and (larp6c3+/-; larp6a-1) mutants. Transmission Efficiency (TE) was calculated as: (([No of mutant] / [No of wild type]) x 100). ns: the number 
Supplemental data. Billey, Hafidh et al. (2021). LARP6C orchestrates post-transcriptional reprogramming of gene expression during hydration to promote pollen tube guidance. Plant Cell.

of mutant seedlings in the progeny is not significantly different from the expected number of mutant seedlings. (G) In vivo scoring of pollen tube behaviors. Wild-type pistils were pollinated in planta with pollen from various homozygous genotypes and the behavior of pollen tubes monitored and scored over a time course: $4 \mathrm{~h}, 8 \mathrm{~h}$ and $24 \mathrm{~h}$ after pollination (HAP). Type I (white bars): targeting and reception are normal, Type II (grey bars): targeting is defective (see Supplemental Figure S3D). The numbers of scored ovules are reported as " $n$ "' in the table below the graphs. p-values were calculated with a t-test, unpaired, ns: non significative, ${ }^{* * *}, p$-value $<0.001$. 
Supplemental data. Billey, Hafidh et al. (2021). LARP6C orchestrates post-transcriptional reprogramming of gene expression during hydration to promote pollen tube guidance. Plant Cell.

\section{A}

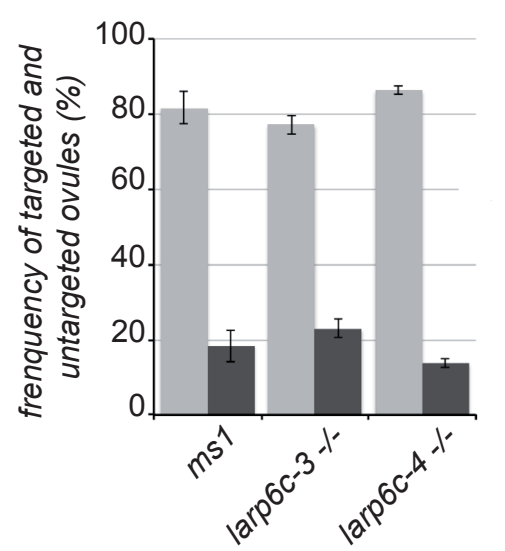

: untargeted

: targeted

B larp6c-4 $\times$ LAT52-GUS
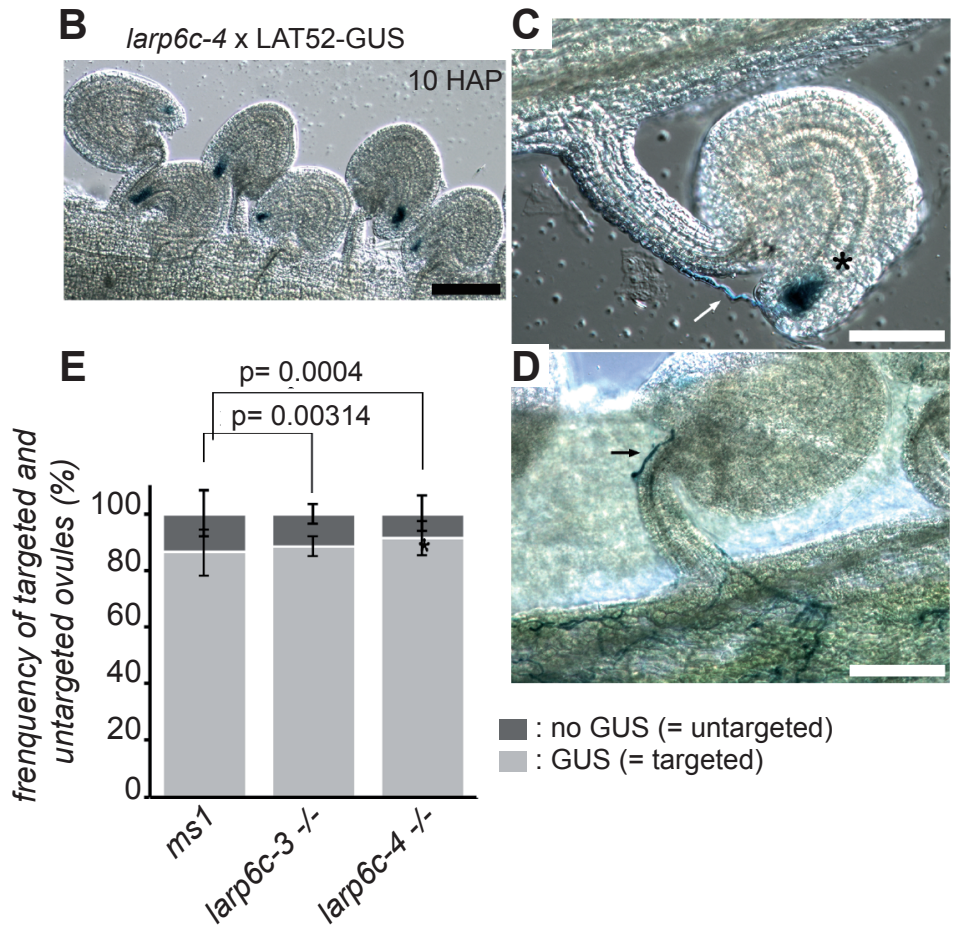

no GUS (= untargeted)

: GUS (= targeted)

Supplemental Figure S4: Pollen attraction competence of larp6c-3 and $6 c 4$ ovules. (Supports Figure 1C-F). (A), Semi in vivo assays using wild-type (WT) (Col 0 ecotype) or larp6c (from larp6c-3 or larp6c-4 homozygous plants) mutant ovules for wild-type (WT) pollen tube attraction. The histograms report the frequency of targeted/untargeted ovules for each ovule genotype. Error bars are standard deviations calculated from 2 biological replicates with $n=150$ ovules (contained in 25 pistil explants) tested for each replicate and each genotype. (B-E), In vivo ovule attraction competence assays. Pollen tube targeting events were scored as successful attraction. ms1, larp6c-3 or larp6c-4 homozygous pistils were pollinated with pollen grains expressing the GUS transgene under the control of the LAT52 promoter. Ovules showing positive GUS staining were scored as competent. (B-D), representative micrographs of pollinated larp6c-4 mutant ovules. Arrows point to pollen tubes (C, D) and asterisks to pollen tube penetration and burst (C). Scale bars correspond to $20 \mu \mathrm{m}$. (E), Histogram representation of ovule attraction frequency. The minus GUS (dark grey) corresponds to frequency of untargeted ovules and the plus GUS (light grey) to the frequency of targeted ovules. Error bars are standard deviations calculated from 2 biological replicates. The total number of ovules scored in replicate $1\left(n_{1}\right)$ and $2\left(n_{2}\right)$ are: $m s 1$ ( $n_{1}=802$ from 24 pistils; $n_{2}=975$ from 29 pistils), larp6c-3 $\left(n_{1}=1063\right.$ from 31 pistils; $n_{2}=869$ from 28 pistils) and larp6c- 4 ( $n_{1}=860$ from 26 pistils, $n_{2}=912$ from 29 pistils). 
Supplemental data. Billey, Hafidh et al. (2021). LARP6C orchestrates post-transcriptional reprogramming of gene expression during hydration to promote pollen tube guidance. Plant Cell.

A
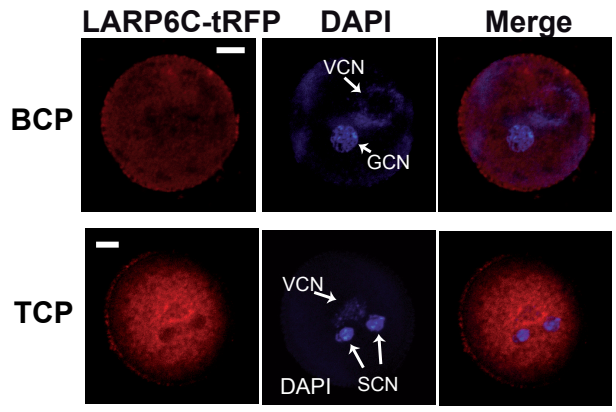

MPG
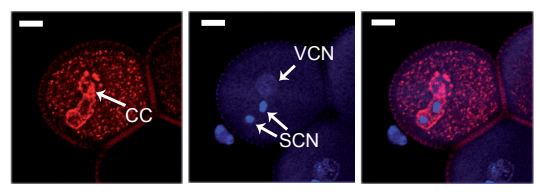

B

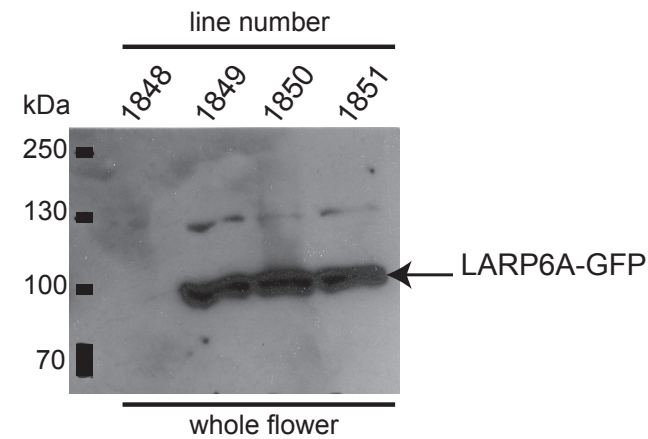

C
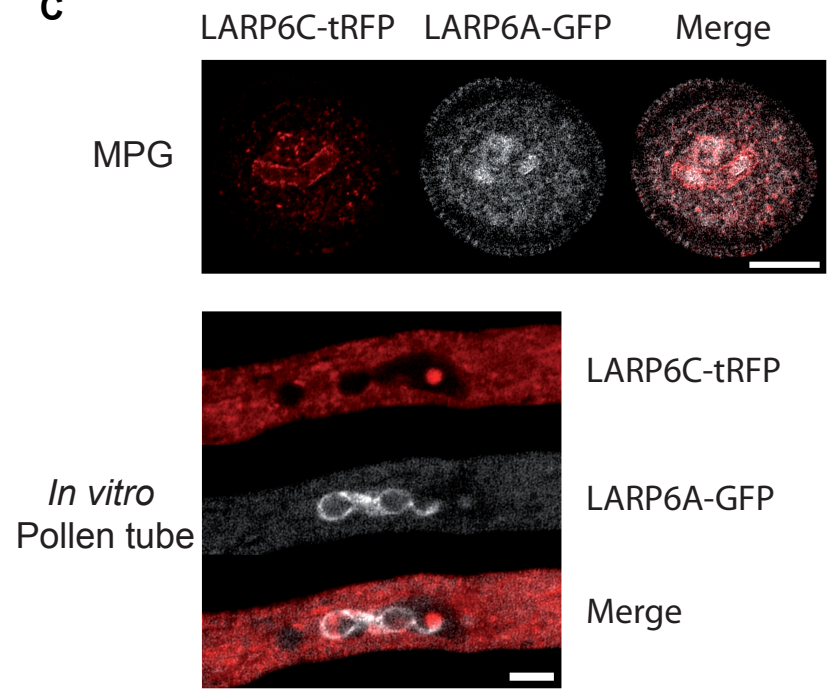

Supplemental Figure S5: Confocal analyses of LARP6C-tRFP and/or LARP6A-GFP in pollen. (Supports Figure 2). (A) Confocal analyses of LARP6C-tRFP distribution in bicellular pollen (BCP), tricellular pollen (TCP), and mature pollen grain (MPG). Line 134 was used to monitor LARP6C-tRFP subcellular distribution. White arrows point to the cytoplasmic connexion (CC), the generative and vegetative cell nuclei (GCN and VCN), and the sperm cell nuclei (SCN). Scale bars correspond to $5 \mu \mathrm{m}$. (B) Immunoblot analysis of LARP6A-GFP expression in four independent transgenic lines. The LARP6A whole genomic region (including promoter and introns) was fused at its C-terminus with the GFP CDS and expressed in the wild-type background. Line 1850 was crossed with line 134 (LARP6C-tRFP) to monitor LARP6A and 6C co-localization. (C) Confocal analysis of LARP6AGFP and LARP6C-tRFP localization in mature pollen grain (MPG) and in vitro grown pollen tubes. Scale bars correspond to 10 $\mu \mathrm{m}$. 
Supplemental data. Billey, Hafidh et al. (2021). LARP6C orchestrates post-transcriptional reprogramming of gene expression during hydration to promote pollen tube guidance. Plant Cell.
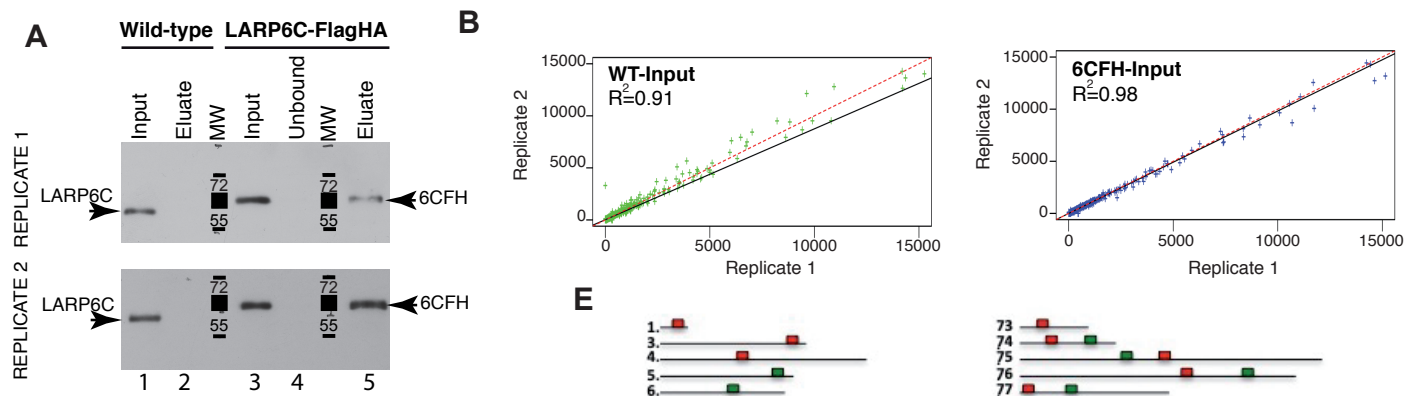

\section{E}

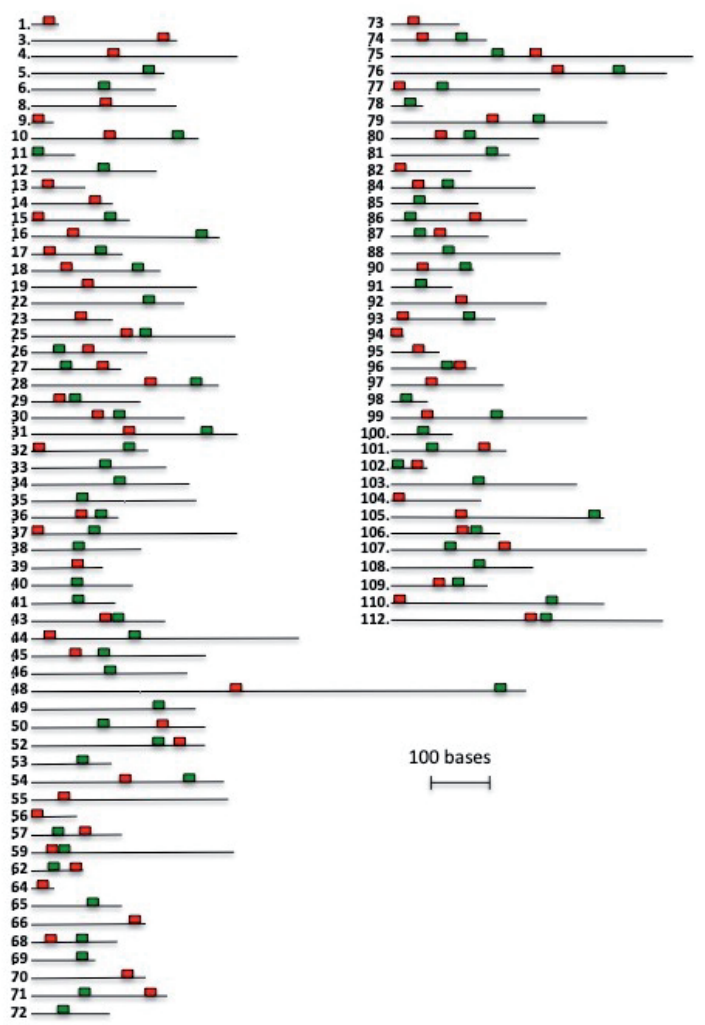

D
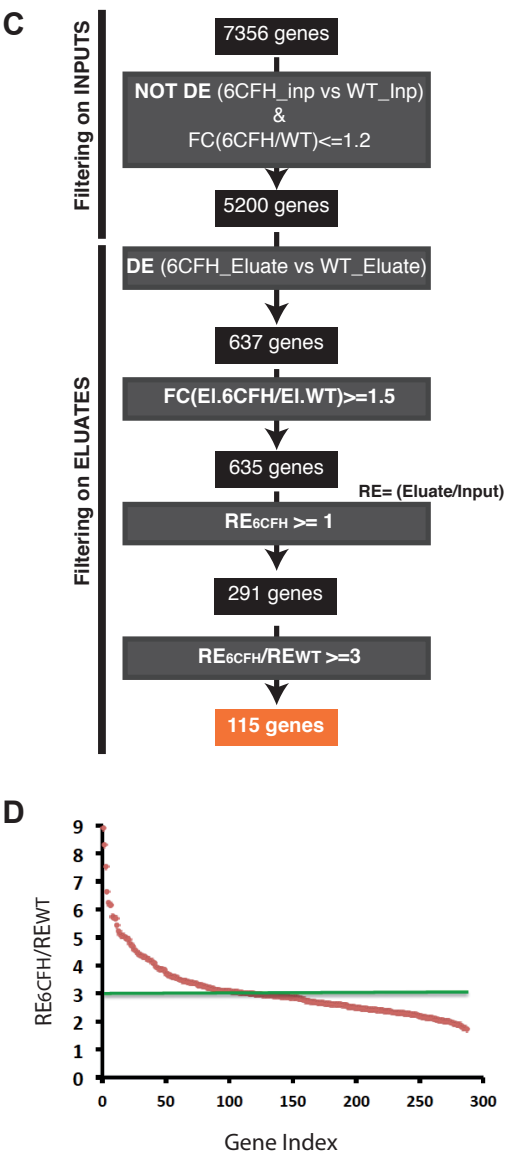

Supplemental Figure S6: Reproducibility of the RIP-seq data, RIP-seq filtering workflow, representation of the position of A and/or B boxes on the 5'-UTRs of LARP6C targets. (Supports Figure 3). (A), Immunoblot analysis of the input (lanes 1, 3), unbound (lane 4), and eluate fractions (lanes 2,5$)$ of the RIP samples. $0.3 \%(15 \mu \mathrm{L})$ of the input and unbound and $3 \%(7.5 \mu \mathrm{L})$ of the eluate fractions were analyzed. The blots were probed with the anti-LARP6C antibody. (B), Comparison of the two biological replicates for the inputs of the RIP-Seq analysis. Correlation of expression levels between replicates of the inputs from wild-type (Col 0) (left panel) or complemented larp6c-3 ((LARP6C-FlagHA; larp6c-3), labelled 6C-FH) (right panel) lines. The X and Y axes correspond to read counts in RPKM respectively for replicates 1 and 2 . The expected correlation line for a correlation coefficient of 1 is represented by a red dotted line and the actual correlation line is in black. Correlation coefficients are reported as $\mathrm{R}^{2}$ on each graph. (C), Representation of the workflow utilized to identify the putative mRNA targets of the LARP6C protein in mature pollen grains. The filtering process was conducted with the weighted average values calculated between replicates, and with the list of genes that displayed at least 1 RPKM value in one of the four conditions (inputs, wild type and 6C-FH and eluates, wild type, and 6C-FH) (see Supplemental Data Set S1). To identify Differentially Expressed (DE) genes between wild type and 6C-FH, respectively, in the input and eluate fractions, we used the Cuffdiff suite. The first filtering step consisted of the elimination of all DE genes in the input. To increase stringency, in the first step, we also filtered out genes non-DE but having Fold Change (FC) values over 1.2, calculated as the ratios between $6 \mathrm{C}-\mathrm{FH}$ and WT inputs. The second filtering step selects DE genes between the eluate fractions that present a FC of 1.5 or more, calculated as the ratios between 6C-FH and WT eluates. The third filtering step is based on the Enrichment Ratio (RE) calculated as the ratio between eluate and input values and initially selected genes with a $\mathrm{RE} 6 \mathrm{CFH} \geq 1$. We then calculated the ratio between RE6C-FH and REWT values, whose distribution is represented in $\mathbf{D}$ and retained genes with a ratio $\geq 3$. The target gene list is shown in Supplemental Data Set $S 1$. (E), Schematic representation of the $5^{\prime}$-UTRs of Box A and/or Box B containing mRNAs identified as putative LARP6C baits. The positions of the A (red boxes) and B (green boxes) motifs are shown. Correspondence between numbers on the left hand-side and gene accession numbers are reported in Supplemental Data Set S1. 
Supplemental data. Billey, Hafidh et al. (2021). LARP6C orchestrates post-transcriptional reprogramming of gene expression during hydration to promote pollen tube guidance. Plant Cell.

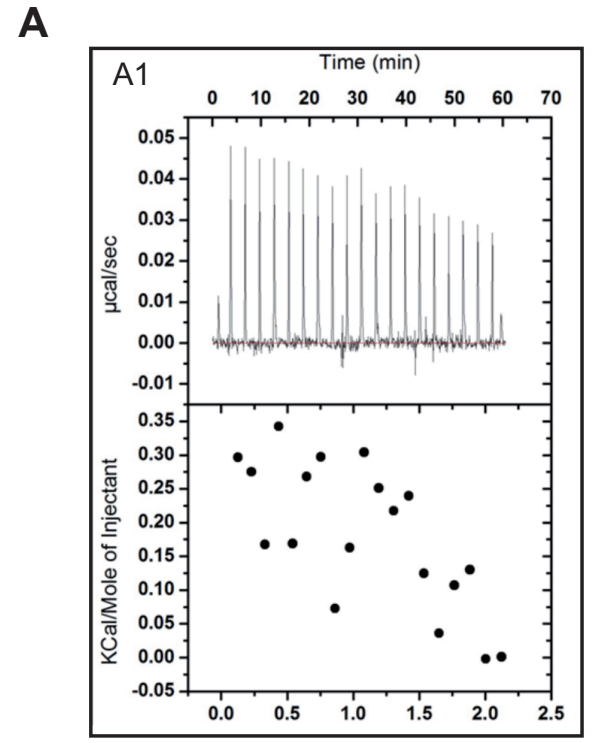

A1: 5'-AAAAAAGAAGAAAAA-3'

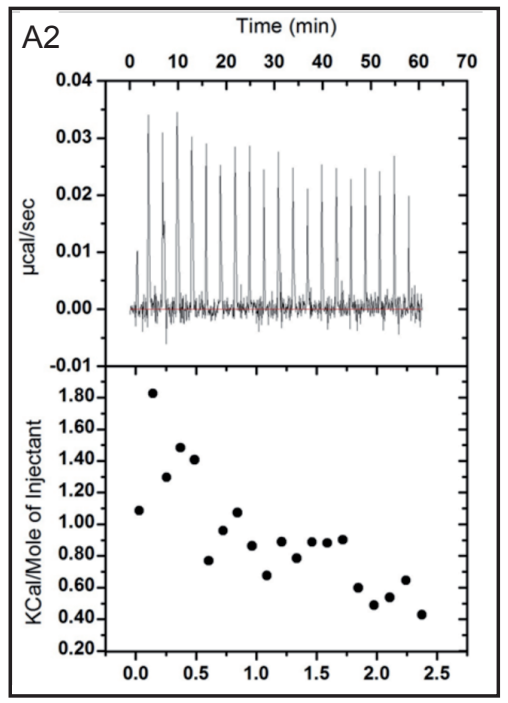

A2: 5'-GAGAGAGGAGAAGAA-3'

B
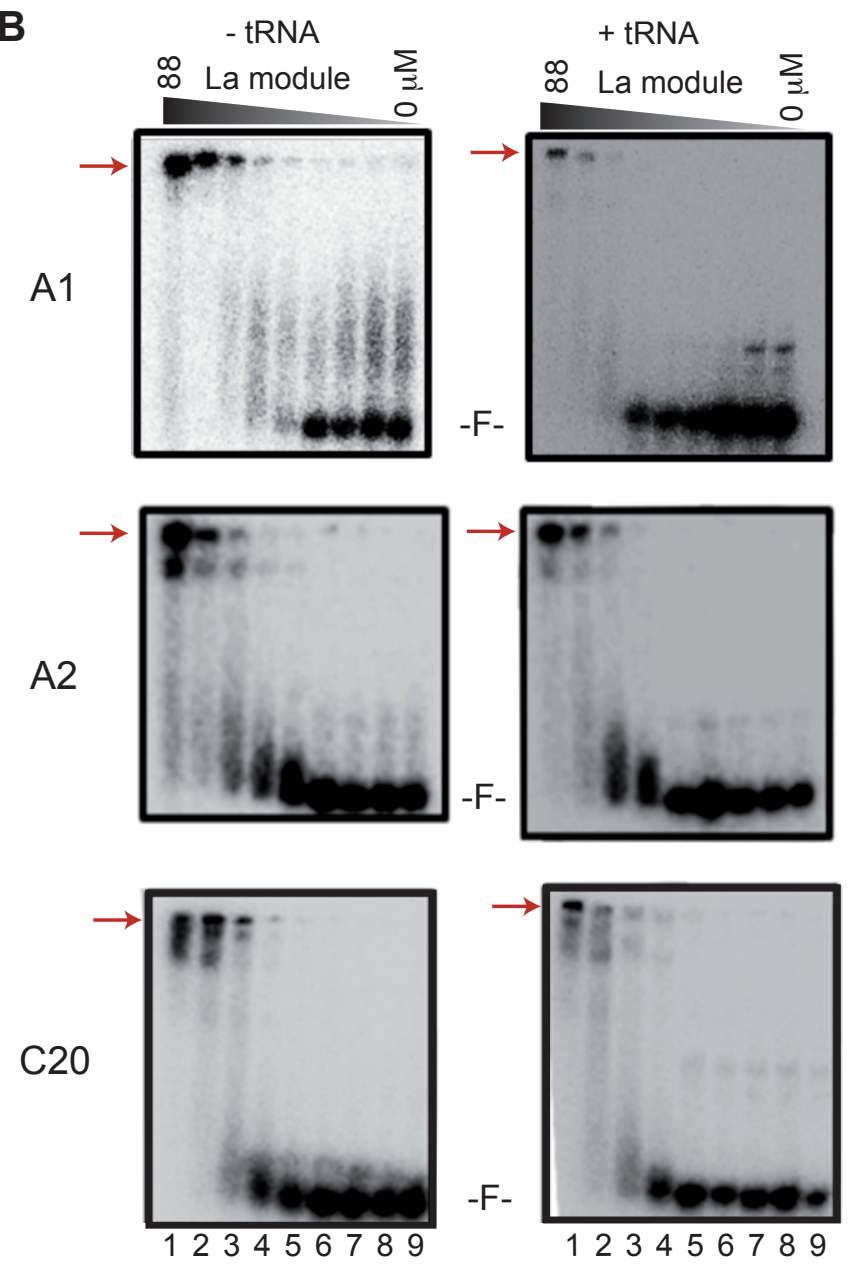

Supplemental Figure S7: ITC and EMSA assessment of LARP6C La-module binding to A type oligos. (Supports Figure 4). (A), Calorimetric analysis of the interaction between the LARP6C La-module (encompassing residues 137-332) and two A-type oligos (A1 and A2) whose sequences are reported below the graphs. For each graph, the upper panel corresponds to the raw titration data showing the thermal effect of injecting an RNA oligos solution into a calorimetric cell containing the recombinant LARP6C La-module. The lower panels show the normalized heat value for the titrations obtained by integrating the raw data and subtracting the heat value of the RNA dilution. (B), EMSAs of the LARP6C La-module binding to A1, A2, or C20 oligos. Decreasing concentrations $(\mu \mathrm{M}) 88$ (lane 1), 29.3 (lane 2), 9.8 (lane 3), 3.3 (lane 4), 1.1 (lane 5), 0.4 (lane 6), 0.12 (lane 7), 0.04 (lane 8) and 0 (lane 9 ) of the recombinant La-module were mixed with $3 \mathrm{nM}$ of 5'-labelled oligos. F stands for Free RNA and the red arrows show samples that were retained in the wells of the gel. Experiments were conducted in the absence (-tRNA) or presence (+tRNA) of unlabelled competitor (tRNAmix of E. coli MRE 600 at $0.01 \mathrm{mg} / \mathrm{mL}$ concentration). 
Supplemental data. Billey, Hafidh et al. (2021). LARP6C orchestrates post-transcriptional reprogramming of gene expression during hydration to promote pollen tube guidance. Plant Cell.

\begin{tabular}{|c|c|c|c|c|c|c|}
\hline Experiment Syringe: Cell & $\mathbf{n}$ & $\begin{array}{c}K_{b} \\
M^{-1}\end{array}$ & $\begin{array}{l}K_{d} \\
\mu M\end{array}$ & $\begin{array}{c}\Delta \mathrm{H} \\
\mathrm{kcal} / \mathrm{mol}\end{array}$ & $\begin{array}{c}-\mathrm{T} \Delta \mathrm{S} \\
\mathrm{kcal} / \mathrm{mol}\end{array}$ & $\begin{array}{c}\Delta \mathrm{G} \\
\mathrm{kcal} / \mathrm{mol}\end{array}$ \\
\hline B1:La module 6C & $0.8+/-0.01$ & $(2.8+/-0.4) \times E^{5}$ & $3.5+/-0.5$ & $-6.6+/-0.1$ & $-1.3+/-0.1$ & $-7.4+/-0.1$ \\
\hline B2:La module 6C & $0.9+/-0.1$ & $(3.7+/-0.9) \times E^{5}$ & $2.8+/-0.4$ & $-6.6+/-0.3$ & $-1.0+/-0.3$ & $-7.6+/-0.1$ \\
\hline B3:La module 6C & na & na & na & na & na & na \\
\hline B4:La module 6C & na & na & na & na & na & na \\
\hline $\mathrm{U}_{20}$ :La module $6 \mathrm{C}^{*}$ & $1.1+/-0.1$ & $(5.8+/-0.3) \times E^{5}$ & $1.7+/-0.1$ & $-3.1+/-0.1$ & $-4.7+/-0.7$ & $-7.8+/-1.0$ \\
\hline A1:La module 6C & na & na & na & na & na & na \\
\hline A2:La module $6 \mathrm{C}$ & na & na & na & na & na & na \\
\hline $\mathrm{C}_{20}$ : La module $6 \mathrm{C}$ & na & na & na & na & na & na \\
\hline
\end{tabular}

Every value is averaged over three experiments and the errors are obtained as the standard deviation from the mean value. *: The values for oligo $\left(U_{20}\right)$ are those previously reported in (Merret et al. RNA, 2013). na: not attributed.

Supplemental Table S1: Thermodynamic parameters of the calorimetric analyses shown in Figure 4B, 4D and Supplemental Figure 6A. 
Supplemental data. Billey, Hafidh et al. (2021). LARP6C orchestrates post-transcriptional reprogramming of gene expression during hydration to promote pollen tube guidance. Plant Cell.
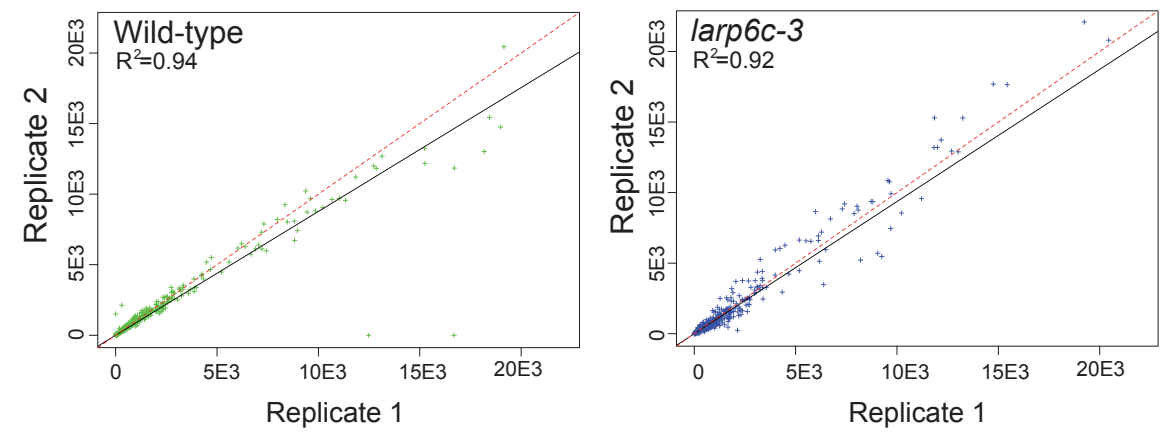

Supplemental Figure S8: Reproducibility of the RNA-seq data. (Supports Figure 7) Comparison of the two biological replicates used for the RNA-seq analyses. Correlation of expression levels between replicates of the RNA-seq data from wild-type (Col 0) (left panel) or from larp6c-3 (right panel) lines. The $\mathrm{X}$ and $\mathrm{Y}$ axes correspond to read counts in RPKM respectively for replicates 1 and 2 . The expected correlation line for a correlation coefficient of 1 is represented by a red dotted line and the actual correlation line is in black. Correlation coefficients are reported as $\mathrm{R}^{2}$ on each graph. 
Supplemental data. Billey, Hafidh et al. (2021). LARP6C orchestrates post-transcriptional reprogramming of gene expression during hydration to promote pollen tube guidance. Plant Cell.

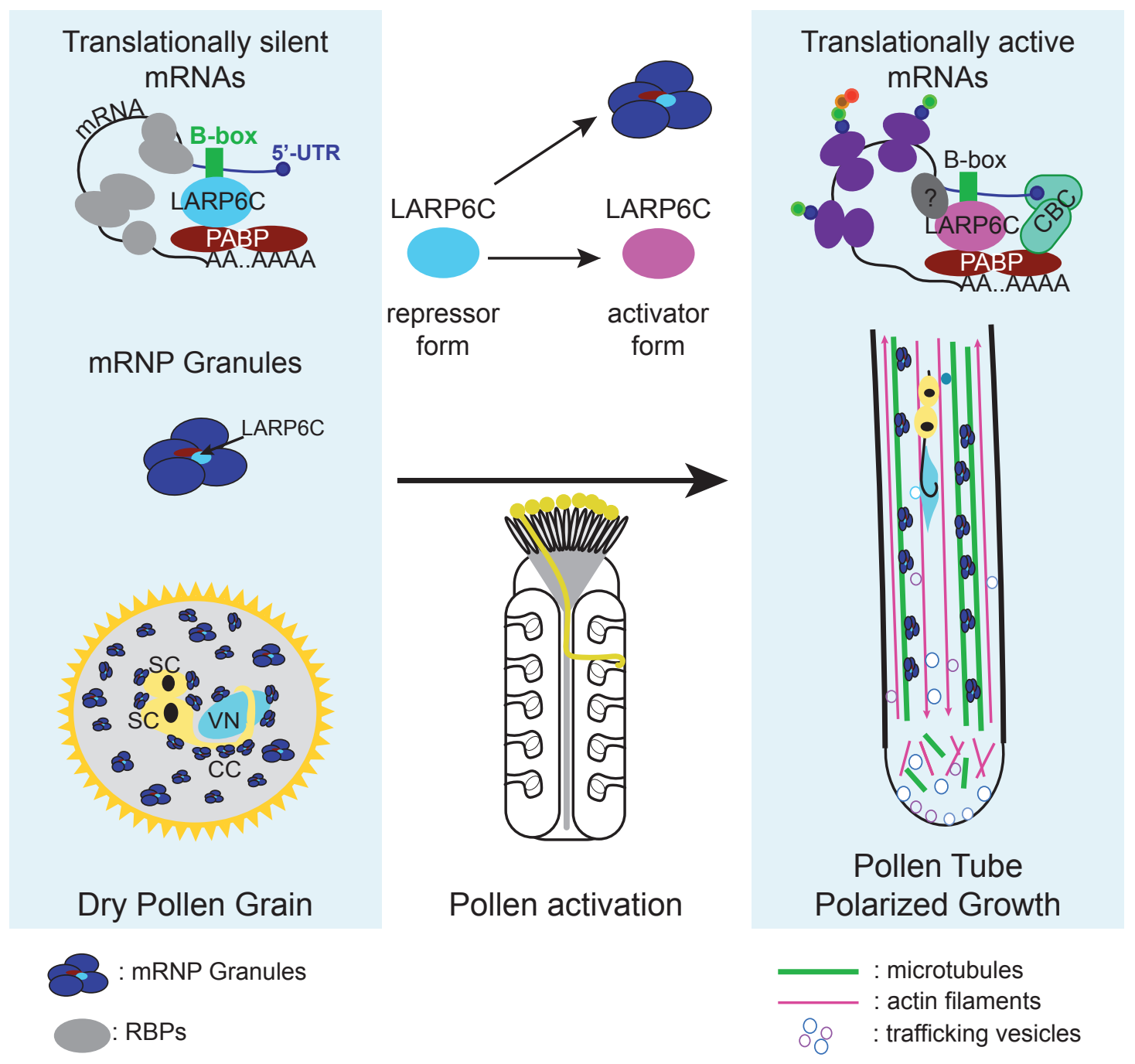

Supplemental Figure S9: Model of the molecular functions of LARP6C in male fertilization. In mature dry pollen grain, LARP6C is part of mRNP granules and acts as $s$ repressor of translation of its target transcripts. During pollen hydration and along the progamic phase, LARP6C shifts from a repressor to an activator of translation. In growing pollen tubes, we postulate that LARP6C could also participate in the transport of at least some of its target transcripts, navigating them along the cytoskeleton as translationally silent to their site of translation. 
Supplemental data. Billey, Hafidh et al. (2021). LARP6C orchestrates post-transcriptional reprogramming of gene expression during hydration to promote pollen tube guidance. Plant Cell.

\begin{tabular}{|c|c|c|c|c|c|c|}
\hline \multicolumn{7}{|c|}{ qPCR PRIMERS } \\
\hline $\begin{array}{l}\text { Primer } \\
\text { Name }\end{array}$ & Gene & 5'-3' sequence & $\begin{array}{l}\text { Primer } \\
\operatorname{Tm}\left({ }^{\circ} \mathrm{C}\right)\end{array}$ & $\begin{array}{c}\text { Amplicon } \\
\operatorname{Tm}\left({ }^{\circ} \mathrm{C}\right)\end{array}$ & $\begin{array}{c}\text { Amplicon } \\
\text { lenght } \\
\text { (nt) }\end{array}$ & $\begin{array}{c}\text { Primer } \\
\text { pair } \\
\text { Efficiency } \\
\text { (\%) }\end{array}$ \\
\hline YFP-F & YFP & TAAACGGCCACAAGTTCAGC & 59.05 & 86.91 & 87 & 99.85 \\
\hline YFP-R & & CGGTGGTGCAGATGAACTTC & 59.20 & & & \\
\hline Hyg-F & Hygromycin & CGTGGTTGGCTTGTATGGAG & 58.91 & 86.91 & 156 & 97.9 \\
\hline Hyg-R & & CCCAAGCTGCATCATCGAAA & 58.90 & & & \\
\hline MGD2-F & YFP-MGD2 & TGTGACTGCATCATCACAAAAG & & & & \\
\hline MGDR & & GGCTGAGCTAGTTTACGTGCAT & & & & \\
\hline $\begin{array}{l}\text { TUBULIN } \\
8-\mathrm{R}\end{array}$ & TUBULIN 8 & CTTCGTATTTGGTCAATCCGGTGC & & & & \\
\hline $\begin{array}{c}\text { TUBULIN } \\
8-\mathrm{F}\end{array}$ & & GAACATGGCTGAGGCTGTCAAGTA & & & & \\
\hline
\end{tabular}

\begin{tabular}{|c|c|c|}
\hline \multicolumn{3}{|c|}{ Cloning \& Genotyping Primers } \\
\hline $\begin{array}{l}\text { Primer } \\
\text { Name }\end{array}$ & 5'-3' sequence & Purpose \\
\hline 31 & CACCtcacacttttcaagtctcatt & \multirow{2}{*}{$\begin{array}{l}\text { MGD2 genomic region } \\
\text { cloning ( }-579 \text { to last } \\
\text { nt before stop codon) }\end{array}$} \\
\hline 32 & AACTAAACTGGCAAAAGAAGAG & \\
\hline 543 & CCCCGGTACCGTGCTTTCTGATTTAAGAGAGGCAAAGGA & \multirow{2}{*}{$\begin{array}{l}\text { LARP6C genomic region } \\
\text { (-1181 to Stop codon) } \\
\text { cloning }\end{array}$} \\
\hline 544 & CCCCGCTAGCGAGATTGTTAGGTGAGAGTGAGATGGAA & \\
\hline 974 & CCCCGAGCTCGTGCTTTCTGATTTAAGAGAGGCAAAGGA & \multirow{2}{*}{$\begin{array}{l}\text { ProLARP6C genomic } \\
\text { region }(-1181 \text { to }-1) \\
\text { cloning }\end{array}$} \\
\hline 975 & CCCCGGTACCTTTCCTCTCACTACTCTGAAAAAATCTCTAAC & \\
\hline 976 & CCCCCACTAGTATGGCGCAGATGCAGCGAGAAG & \multirow{2}{*}{$\begin{array}{l}\text { Cloning of genomic } \\
\text { LARP6C from ATG to } \\
\text { STOP codon }\end{array}$} \\
\hline 977 & CCCCCGATATCTTAGAGATTGTTAGGTGAGAGTGAGATGG & \\
\hline eb3 & СCCCTCTAGACTCGAGCTAGAAAGAGAACCAGGGACTTCT & \multirow{2}{*}{$\begin{array}{l}\text { Cloning of genomic } \\
\text { PAB5 from ATG to STOP } \\
\text { codon }\end{array}$} \\
\hline eb4 & CCCCGGATCCCTCGGATGAGGATAGAGCAAACTGGTCATC & \\
\hline 1197 & GCAGGCTGCGGCCGCTCTAG & \multirow{2}{*}{$\begin{array}{l}\text { Construction of } \\
\text { LARP6CDLAM CDS } \\
\text { (underlined sequence } \\
\text { is complementary to } \\
\text { the } 20 \text { first nt of } \\
\text { pcr product 2, } \\
\text { obtained with primers } \\
1198 \text { and } 1200 \text { ) }\end{array}$} \\
\hline 1198 & GCAATTCCTCTCTGTCTCTCTTCGACGAAGAAGACGAGTTCTGA & \\
\hline
\end{tabular}


Supplemental data. Billey, Hafidh et al. (2021). LARP6C orchestrates post-transcriptional reprogramming of gene expression during hydration to promote pollen tube guidance. Plant Cell.

\begin{tabular}{|l|l|l|}
\hline 1199 & AGAGACAGAGAGGAATTGCA & $\begin{array}{l}\text { Construction of } \\
\text { LARP6CDLAM CDS }\end{array}$ \\
\hline 1200 & TATCGATAAGCTTGATATCGA & \\
\hline 1201 & GCTCTAGAATGGCGCAGAT & larp6c-3 LP primer \\
\hline 488 & GAAGTAGAGTCCGTCACGACG & larp6c-3 RP primer \\
\hline 489 & TTTCTTGCCGTCTTCACTCAC & SAIL-LB2 \\
\hline 491 & TAGCATCTGAATTTCATAACCAATCTCGATACAC & larp6c-4 LP primer \\
\hline 1099 & GTTCAAATTCAACGCTCAAGC & larp6c-4 RP primer \\
\hline 483 & AATTCTCTGCAACGACAGTCC & DsLox-p745-LB \\
\hline 475 & AAGCTGGAAGGTAGGCTATGG & larp6a-1 RP primer \\
\hline 476 & GACGCTTTGAGACCTCAACAG & larp6a-1 LP primer \\
\hline 474 & ATACGACGGATCGTAATTTGTG & Saskatoon-3 \\
\hline
\end{tabular}

Supplemental table S2: List and sequences of primers used for qPCR analyses, genotyping and cloning. 


\section{SUPPLEMENTAL METHODS}

\section{Plant material and plasmid construction}

The larp6a-1 (SK39668) line from the Saskatoon Arabidopsis T-DNA population collection (Robinson et al., 2009) was obtained from the NASC stock center. Genotyping primer sequences are listed in Supplemental Table S2. To determine whether this insertion line was loss-of-function, we ordered a custom-made antibody through immunization of rabbits with peptide "EESEKGGKKENFIRTR" (Agro-Bio (La Ferté St Aubin, France)). Anti-LARP6A antibodies were used at 1/5,000 dilution. To obtain the LARP6A-GFP fusion, we PCR amplified the whole genomic locus AT5G 46250 from position -1498 up to the last nucleotide before the stop codon with primers 959 and 960 and inserted the DNA fragment following Kpnl-Sacl digestion upstream of the GFP, into a pCAMBIA1300-based plant binary vector expressing the HPTII (hygromycin) resistance gene, giving rise to plasmid p791. A stable transgenic line was prepared through agroinfiltration of plasmid p791 into wild-type Col-0. To monitor co-localization of LARP6A and LARP6C, we transferred the LARP6A-GFP transgene (line 1850) into a LARP6C-tRFP expressing line (line 134) through crossing.

\section{Growth conditions and pollen collection technique for RNA-Seq and RIP-Seq assays}

Mature pollen materials for RNA-seq and RIP-seq were obtained from plants sown, grown and harvested under the exact same conditions. Wild type (Col-0), larp6c-3 and (larp6c-3; $\mathrm{PRO}_{6 \mathrm{C}-}$ LARP6CFLAGHA) (abbreviated as 6C-FH) were sown on $1 / 2 \mathrm{MS}-0.8 \%$ agar in duplicate and cultivated for 15 days in vitro at $20^{\circ} \mathrm{C}$, with $24 \mathrm{~h}$ light $\left(50-60 \mathrm{mE}^{-1} \mathrm{~m}^{-2}-\mathrm{s}^{-1}\right)$. Seedlings were then transferred to soil treated with the nematode Steinernema feltiae (at $5000 /$ soil Liter) and cultivated in a greenhouse with approximately $16 \mathrm{~h}$ day at $22^{\circ} \mathrm{C}$ night and day (greenhouse conditions, July 2013 Gif/Yvette France) for 5 weeks. Wild type Col-0, larp6c-3 and 6C-FH (transformant number 267 (see Supplemental Figure S3C for an immunoblot analysis of LARP6CFLAGHA protein accumulation)) plants were grown together in the same compartment of the greenhouse in large plates, each containing 96 plants. Two sets (Col-0, larp6c-3, 6C-FH) of plants were grown to produce two replicates. For each replicate, $15 \mathrm{Col}-0,3$ larp6c-3 and $156 \mathrm{C}-\mathrm{FH}$ plates were prepared. The plates of each replicate were respectively installed on two distinct growth shelves inside a same compartment and each genotype interspersed inside each replicate. To avoid the effects of cooling air efflux and temperature gradients and to obtain homogeneous growth between genotypes inside each replicate, the plates were rotated along their respective shelf every 2 weeks. Mature pollen grains were collected using a homemade pollen collector as described in (Johnson-Brousseau and McCormick, 2004). Briefly, mature pollen was collected by aspiration with a vacuum cleaner equipped with three successive filters 
of 140,60 and $10 \mu \mathrm{m}$ diameter. Pollen grains were scraped from the $10 \mu \mathrm{m}$ filter into an Eppendorf tube, weighed, flash frozen in liquid nitrogen, and stored at $-80^{\circ} \mathrm{C}$.

\section{RNA sequencing}

Total RNAs were extracted from $5 \mathrm{mg}$ of wild-type and larp6c-3 mature pollen grains as described in the Methods section of the main text. RNA quality was verified by gel electrophoresis and staining with Gel-red (Biotium). DNase treatment was conducted as for RT-PCR assays. The reaction was stopped and DNase extracted with phenol/chloroform/IAA before RNA was precipitated and resuspended in RNase-free water. The quality of DNase-treated RNA was assessed with a Bio-Analyser. Library preparation (with a True seq Stranded mRNA kit from Illumina) and sequencing were subcontracted to Fasteris (Switzerland). Single-end (101bp read length) sequencing was performed on the HiSeq 2000 platform with a depth of 31 to $60 \mathrm{M}$ reads. Experiments were conducted in duplicate for each genotype.

\section{RNA Immunoprecipitation and sequencing (RIP-Seq)}

After pollen collection, crude extracts (Inputs) were prepared from identical amounts of wild-type and $6 \mathrm{C}-\mathrm{FH}$ expressing pollen grains. Twenty-five $\mathrm{mg}$ of mature pollen was resuspended in 250 volumes of extraction buffer (50 mM Tris $\mathrm{HCl} \mathrm{pH} \mathrm{7.4,10 \%} \mathrm{glycerol,} 100 \mathrm{mM} \mathrm{NaCl}, 5 \mathrm{mM} \mathrm{MgCl}$, 80 units / ml RNAsin $®$ Plus RNAse Inhibitor (Promega), 1X mg132, 1\% protease inhibitor cocktail for plant extract (Sigma)) and ground using a Silamat S6 before extracts were cleared by centrifugation $\left(4^{\circ} \mathrm{C}, 14.000 \mathrm{~g}\right.$ for $10 \mathrm{~min}$ ). 700 and $40 \mu \mathrm{L}$ of Input fraction were saved to purify total RNA (Input RNA) and total protein (Input Protein) to respectively conduct RNA-sequencing and immunoblotting. For each sample (Col0-Replicate 1, Col0-Replicate 2, 6C-FH-Replicate 1 and $6 \mathrm{C}-\mathrm{FH}$-Replicate 2), the immunoprecipitation step was conducted with $5 \mathrm{~mL}$ of crude extract. For each sample, $250 \mu \mathrm{L}$ of Anti-Flag M2 (Sigma) magnetic beads were thoroughly washed with 10 volumes of extraction buffer and evenly divided between the 5 tubes. After removing the supernatant, $1 \mathrm{~mL}$ of crude extract (approximately $300 \mu \mathrm{g}$ of total protein) were added to each tube and rotated for $1 \mathrm{~h}$ at $4^{\circ} \mathrm{C}$ on a wheel at $10 \mathrm{rpms}$. The unbound fractions were collected, pooled, and 700 and $60 \mu \mathrm{L}$ stored for RNA and protein analyses. The beads were washed once with 5 vol of washing buffer ( $50 \mathrm{mM}$ Tris $\mathrm{HCl} \mathrm{pH} \mathrm{7.4,} 10 \%$ Glycerol, $150 \mathrm{mM} \mathrm{NaCl}, 3 \mathrm{mM} \mathrm{MgCl}$, 80 units / ml RNAsin® Plus RNAse Inhibitor (Promega), 1X Mg132, 1\% protease inhibitor cocktail for plant extract (Sigma), $0.1 \% \operatorname{Triton}^{\mathrm{TM}}$ (Sigma)) before being transferred to a new tube, pooled, and washed twice more in $2 \mathrm{vol}$ of washing buffer. The elution step was conducted as follow: $6 \%$ of the beads $(15 \mu \mathrm{L})$ were resuspended in $1 \mathrm{X}$ Laemmli buffer, heated for $10 \mathrm{~min}$ at $100^{\circ} \mathrm{C}$, and 
the supernatant used to run immunoblot analysis to monitor the efficiency of immunoprecipitation of the $6 \mathrm{C}-\mathrm{FH}$ protein. The remaining $94 \%$ of the beads were resuspended into $400 \mu \mathrm{L}$ of guanidium hydrochloride buffer, incubated for $10 \mathrm{~min}$ at RT, the supernatant collected and separated from proteins with two phenol/chloroform/IAA extractions before the RNA was precipitated in the presence of $80 \mu \mathrm{g}$ of glycogen and resuspended into $10 \mu \mathrm{L}$ of RNase-free water. RNAs in the eluate fractions were quantified with a NanoDrop and their quality verified with a Bio-Analyzer system. The library preparation and sequencing (Input RNA and Eluate RNA) were subcontracted to Fasteris (Switzerland). The libraries constructed with the input RNAs were prepared from poly(A) purified RNAs using a True seq Stranded mRNA kit from Illumina and the libraries prepared from the eluate fractions were constructed from unpurified RNAs. Sequencing was performed as single-end $101 \mathrm{bp}$ and with a depth of 42 to $64 \mathrm{M}$ reads for the inputs and 25 to $40 \mathrm{M}$ reads for the eluates.

\section{Bioinformatics}

For each RNA-seq and RIP-seq sample and replicate, read quality was first verified with FastQCsoftware (http://www.bioinformatics.babraham.ac.uk/projects.fastqc) and clusters with a Qscore below 30 filtered out. 95-96\% of the RNA-seq clusters and $92-94 \%$ of the RIP-seq clusters had a Qscore of at least 30 (please see Supplemental Data Set S1 and S2 for the sequencing depth and quality scores of each sample). In the second step, non-nuclear genomic and ribosomal RNA sequences were filtered out against the TAIR10 database using Bowtie2 (Langmead and Salzberg, 2012) and the remaining clusters aligned against TAIR10 (TAIR10_genes_transposons.gtf) with TopHat2 (Kim et al., 2013) . After filtering, more than $99 \%$ of the clusters were retained for the RNA-seq samples and inputs of the RIP-seq. For the eluates, $5 \%$ of the Col- 0 clusters and $15 \%$ of the $6 \mathrm{C}-\mathrm{FH}$ clusters were retained, because of the high contamination with ribosomal RNAs. For the RNA-seq and RIP-seq assays, genes that did not display at least 1 RPKM (read per kilobase per million mapped reads) value in one of the conditions were filtered out. Normalized mean values were attributed to each gene, and differentially expressed genes were identified with the Cufflinks, Cuffmerge and Cuffdiff suite (Trapnell et al., 2010) with an FDR of 0.05 for RNA-seq and 0.01 for RIP-seq and $|\log 2(F C)| \leq$ 0.585 as cut-off for total RNA-seq and $\log 2(F C) \geq 0.585$ for eluate values of RIP-Seq. To search for a motif shared by putative targets of LARP6C, we looked at the Araport database (https://www.araport.org/) for their 5' and 3'-UTR sequences and retrieved sequences for 112 out of 115 putative targets. We then ran MEME searches (at the MEME suite portal (http://meme- 
suite.org/)) using, as control sequences, a set of 112 randomly chosen 5'- and 3'-UTR sequences from genes expressed in pollen according to our transcriptomic analysis.

\section{Pollen phenotyping: pollen maturation, pollen tube germination and growth}

To monitor pollen maturation defects, mature pollen grains were collected from wild-type and larp6c homozygous plants, deposited on a DAPI solution (1X PBS pH7, 0.5\% Triton, $1 \mu \mathrm{g} / \mathrm{mL}$ 4',6-diamidino-2-phenylindole (DAPI)) and the number of mutant pollen grains with undivided generative cells determined using confocal microscopy. Pollen tube germination and growth defects were monitored in vitro or through semi in vivo assays. To grow pollen tubes in vitro, we used a modified version of the protocol from (Boavida and McCormick, 2007). Freshly prepared pollen tube growth medium $\left(0.01 \%\right.$ boric acid, $5 \mathrm{mM} \mathrm{CaCl}_{2}, 5 \mathrm{mM} \mathrm{KCl}, 1 \mathrm{mM} \mathrm{MgSO} 4,10 \%$ sucrose, $\mathrm{pH} 7.5(\mathrm{KOH}), 1.5 \%$ Phytagel) was directly poured onto a microscope slide. Mature pollen was deposited on the solid medium and slides incubated for $8 \mathrm{~h}$ in a humid chamber at 20$22^{\circ} \mathrm{C}$ in the dark. Pollen tubes were photographed (with a Hamamatsu camera (Sunayama-cho, Shizuoka, Japan)) and pollen tube lengths measured with NIS-element software from Nikon (Nikon Instruments, Melville, NY USA). Pollen tube density and length were also determined through semi in vivo analyses. Following hand pollination with wild-type or larp6c homozygous mutant pollen, the pistils were excised at the shoulder and incubated for $16 \mathrm{~h}$ on a plate containing pollen tube solid growth medium. Pistil explants and pollen tubes were photographed and images analyzed with the plugin Bio-format (Open microscopy environment) from Image $\mathrm{J}$ software (Rasband, W.S. ImageJ, NIH, Bethesda USA) (Abràmoff et al., 2004) to determine pollen tube length and density per pistil.

\section{Ovule attraction assays}

larp $6 c-3$ or larp $6 c-4$ pistils were emasculated at stage $12 \mathrm{C}$ and left to mature for 2 days. Limited pollination was performed on day 3 using LAT52-GUS homozygous pollen grains in the qrt-/background alongside ms1-/- pistils as the control. Eighteen hours after pollination, pollinated pistils were collected and gently dissected with a $25 \mathrm{G}$ needle to expose fertilized ovules. Dissected pistils were stained for GUS activity in GUS staining buffer (50 mM PBS, pH7, 0.2\% Triton-X100, $10 \mathrm{mM}$ potassium ferrocyanide and $1 \mathrm{mM}$ X-Glu). Pistils were vacuum infiltrated for $10 \mathrm{~min}$ and stained $\mathrm{o} / \mathrm{n}$ at $37^{\circ} \mathrm{C}$. For microscopy analyses, stained pistils were cleared using an ethanol series (70\%-30\%), mounted on 30\% glycerol, and analyzed with a Nikon TE2000 equipped with DIC modulation contrast and NIS-element software. Images were processed with ImageJ. 


\section{SUPPLEMENTAL REFERENCES}

Abràmoff, M., Magalhaes, P., and Ram, S. (2004). Image processing with ImageJ. Biophotonics Int. 11: 36-41.

Boavida, L.C. and McCormick, S. (2007). TECHNICAL ADVANCE: Temperature as a determinant factor for increased and reproducible in vitro pollen germination in Arabidopsis thaliana. Plant J. 52: 570-582.

Johnson-Brousseau, S.A. and McCormick, S. (2004). A compendium of methods useful for characterizing Arabidopsis pollen mutants and gametophytically- expressed genes. Plant J. 39: $761-775$.

Kim, D., Pertea, G., Trapnell, C., Pimentel, H., Kelley, R., and Salzberg, S.L. (2013). TopHat2: accurate alignment of transcriptomes in the presence of insertions, deletions and gene fusions. Genome Biol. 14: R36.

Klepikova, A. V, Logacheva, M.D., Dmitriev, S.E., and Penin, A.A. (2015). RNA-seq analysis of an apical meristem time series reveals a critical point in Arabidopsis thaliana flower initiation. BMC Genomics 16: 466.

Langmead, B. and Salzberg, S.L. (2012). Fast gapped-read alignment with Bowtie 2. Nat. Methods 9: 357-359.

Robinson, S.J. et al. (2009). An archived activation tagged population of Arabidopsis thaliana to facilitate forward genetics approaches. BMC Plant Biol. 9: 101.

Trapnell, C., Williams, B.A., Pertea, G., Mortazavi, A., Kwan, G., van Baren, M.J., Salzberg, S.L., Wold, B.J., and Pachter, L. (2010). Transcript assembly and quantification by RNASeq reveals unannotated transcripts and isoform switching during cell differentiation. Nat. Biotechnol. 28: 511-515.

Winter, D., Vinegar, B., Nahal, H., Ammar, R., Wilson, G. V., and Provart, N.J. (2007). An "Electronic Fluorescent Pictograph" Browser for Exploring and Analyzing Large-Scale Biological Data Sets. PLoS One 2: e718. 